\title{
Eulerian and Lagrangian means in rotating, magnetohydrodynamic flows II. Braginsky's nearly axisymmetric dynamo
}

\author{
ANDREW M. SOWARD $\nmid \S^{*}$ and PAUL H. ROBERTS $\ddagger$ \\ $†$ School of Mathematics and Statistics, Newcastle University, Newcastle upon Tyne NE1 7RU, UK \\ $\S$ Mathematics Research Institute, University of Exeter, Exeter, EX4 4QF, UK \\ $\ddagger$ Department of Mathematics, University of California, Los Angeles, CA 90095, USA \\ (Received 25 April 2013; in final form 24 October 2013; first published online ????)
}

\begin{abstract}
The hybrid Euler-Lagrange (HEL) approach has been usefully applied to weakly dissipative systems characterised by waves riding on mean flow. Soward (Phil. Trans. R. Soc. Lond. A, 1972, 272, 431) showed how the HEL-formulation could elucidate remarkable features of the nearly axisymmetric large magnetic Reynolds number dynamo of Braginsky (JETP, 1964, 47, 1084). Since Braginsky's treatment of the nearly axisymmetric dynamo relies on azimuthal averages, those can only be taken when the azimuth is a coordinate direction. In that respect, the unified derivation and presentation of the HEL-equations governing rotating magnetohydrodynamic convective flows, as later reviewed and extended by Roberts and Soward (Geophys. Astrophys. Fluid Dyn. 2006, 100, 457), suffers the shortcoming that it was developed relative to rectangular Cartesian coordinates. Here we undertake those modifications needed to transform the rectangular Cartesian coordinate formulation into cylindrical polar coordinates. Being a Lagrangian description, application of the HEL-method means that the variables used, dependent on coordinates $\boldsymbol{x}$, do not describe conditions at the position P: $\boldsymbol{x}$ but on conditions elsewhere at some displaced position $\mathrm{P}^{L}$ : $\boldsymbol{x}^{L}(\boldsymbol{x}, t)=\boldsymbol{x}+\boldsymbol{\xi}(\boldsymbol{x}, t)$, generally dependent on time $t$. To address this issue Soward and Roberts (J. Fluid Mech., 2010, 661, 45) invoked an idea pioneered by Moffatt (J. Fluid Mech., 1986, 166, 359), whereby the point $\boldsymbol{x}$ is dragged to $\boldsymbol{x}^{L}(\boldsymbol{x}, t)$ by a 'fictitious steady flow' $\boldsymbol{\eta}(\boldsymbol{x}, t)$ in a unit of 'fictitious time'. This is the 'Lie dragging' technique of general tensor calculus, which we apply here to the HEL-equations governing Braginsky's nearly axisymmetric dynamo. We consider the 'effective-variables' introduced by Braginsky, appropriate for small displacement $\boldsymbol{\xi}$, and show that $\boldsymbol{\eta}$, rather than $\boldsymbol{\xi}$, is their natural expansion variable. As well as revisiting Braginsky's kinematic dynamo, we reassess the hydromagnetic extensions of Tough and Roberts (Phys. Earth Planet. Inter., 1968, 1, 288).
\end{abstract}

Keywords: Kinematic and hydromagnetic dynamos; Hybrid Euler-Lagrange method; Azimuthal averages

\section{Introduction}

A significant way of representing the effects of wave motion on a mean flow was pioneered largely by Andrews and McIntyre (1978a,b) (see also Bühler 2009). They used a Hybrid Euler-Lagrange or HEL-representation, so-called because it provides a compromise between the familiar Eulerian and Lagrangian representations. Its introduction into fluid mechanics is often attributed to Eckart (1963) although some of the essential ideas had previously been advanced by Frieman and Rotenberg (1960). Nevertheless, the nearly axisymmetric dynamo proposed by Braginsky (1964a,b), as interpreted by Soward (1972), provides the paradigm for the HEL-method. A unified derivation and presentation of the HEL-equations governing rotating magnetohydrodynamic convective flows was reviewed and extended by Roberts and Soward (2006a) (henceforth referred to as Part I). We say that Braginsky's nearly axisymmetric dynamo provides the paradigm for the HEL-approach because the advection of a magnetic (vector) field in large magnetic Reynolds number $R m$ flows leads to a transparent illustration of the power of the HEL-method. Similar advantages are gained in application of HEL to the

Corresponding author. Email: andrew.soward@ncl.ac.uk 
advection of heat (scalar) at large Péclet number $P e$, but there its usefulness is less obvious. Indeed, the application of HEL to the momentum equation itself causes complications which lead to additional terms such as the pseudomomentum identified by Andrews and McIntyre (1978a,b).

Being a semi-Lagrangian theory, HEL involves a Lagrangian displacement $\boldsymbol{\xi}$. Recently, building on the pioneering study of Moffatt (1986), Soward and Roberts (2010) invoked a fictitious (or Eulerian) flow $\boldsymbol{\eta}$ to realise the HEL-displacement $\boldsymbol{\xi}$. Thereby HEL-theory is recast in an Eulerian setting, for which Soward and Roberts (2010) coined the term Eulerian Transformed HEL (or simply ETHEL). For any small amplitude displacement theory ETHEL is the natural framework to undertake asymptotic analysis being closely linked to classical Eulerian developments such as those of Braginsky (1964a,b). As such they are readily implemented in asymptotic theories. On the one hand, whereas HEL provides considerable physical insight and is useful as a diagnostic tool, it is difficult to use to actually solve problems. On the other hand, the direct Eulerian approach, such as Braginsky's, is itself difficult to implement because the pertinent (i.e. useful) Eulerian averaged equations are hard to construct. In this introduction, we explain carefully Braginsky's Eulerian approach, which relied on his use of so-called 'effective-variables'. In turn, this pioneering study points to the usefulness of the alternative HEL-approach, from which ETHEL overcomes the enormous problems faced by Braginsky's direct Eulerian construction.

The purpose of this extended introduction is to emphasise the Eulerian objective. The remainder of the paper is concerned with how that may be reached using Lagrangian methods.

\subsection{Mean-field equations}

We emphasise at the outset that our ultimate concern is with the derivation of mean-field equations appropriate to situations involving strong large scale mean flows. Nevertheless, we begin by discussing basic issues common to all mean-field theories. Firstly, we focus on the advection of a vector field exemplified by the magnetic field $\boldsymbol{b}^{*}$ governed the Faraday's, Ohm's, Ampère's and Gauss' laws

$$
\begin{aligned}
\partial_{t} \boldsymbol{b}^{*} & =-\boldsymbol{\nabla} \times \boldsymbol{E}^{*}, & \boldsymbol{E}^{*} & =-\boldsymbol{u}^{*} \times \boldsymbol{b}^{*}+\boldsymbol{J}^{*}, \\
\boldsymbol{j}^{*} & =\boldsymbol{\nabla} \times \boldsymbol{H}^{*}, & \boldsymbol{\nabla} \cdot \boldsymbol{b}^{*} & =0,
\end{aligned}
$$

where $\boldsymbol{u}^{*}$ and $\boldsymbol{E}^{*}$ are the flow velocity and electric field respectively. Also the magnetic field $\boldsymbol{b}^{*}$ and the electric current $\boldsymbol{j}^{*}$ are

$$
\boldsymbol{b}^{*}=\mu \boldsymbol{H}^{*}, \quad \boldsymbol{j}^{*}=\sigma \boldsymbol{J}^{*}, \quad \boldsymbol{J}^{*}=\mathfrak{K} \boldsymbol{\nabla} \times \boldsymbol{b}^{*} \quad \text { with } \quad \mathfrak{K}=(\sigma \mu)^{-1},
$$

in which $\mu, \sigma$ and $\mathfrak{K}$ are the magnetic permeability, electrical conductivity and magnetic diffusivity respectively. Secondly, we consider the advection of scalar fields as exemplified by the equations governing mass continuity:

$$
\partial_{t} \rho^{*}=-\nabla \cdot \boldsymbol{m}^{*}, \quad \text { where } \quad \boldsymbol{m}^{*} \equiv \rho^{*} \boldsymbol{u}^{*}
$$

is the mass flux of the fluid, density $\rho^{*}$ and heat conduction:

$$
\partial_{t} \theta^{*}+\boldsymbol{u}^{*} \cdot \nabla \theta^{*}=\kappa \Delta \theta^{*}+q^{*}, \quad \Delta \equiv \nabla^{2},
$$

where $\theta^{*}$ and $\kappa$ are the temperature and thermal diffusivity, while $q^{*}$ is a possible heat source.

We decompose our various vector and scalar fields into their mean and fluctuating parts:

$$
\boldsymbol{u}^{*}=\overline{\boldsymbol{u}^{*}}+\boldsymbol{u}^{* \prime}, \quad \boldsymbol{b}^{*}=\overline{\boldsymbol{b}^{*}}+\boldsymbol{b}^{* \prime}, \quad \rho^{*}=\overline{\rho^{*}}+\rho^{* \prime}, \quad \theta^{*}=\overline{\theta^{*}}+\theta^{* \prime},
$$

where their respective fluctuations $\boldsymbol{u}^{* \prime}, \boldsymbol{b}^{* \prime}$ and $\rho^{* \prime}, \theta^{* \prime}$ often describe small scale turbulence; the remaining mean parts are identified by the 'bar'. The mean and fluctuating parts of the 
linear equations (1a,c,d) and (2a) are obvious. With regard to the nonlinear equations, the mean part of Ohm's law (1b) gives

$$
\overline{\boldsymbol{E}^{*}}=-\overline{\boldsymbol{u}^{*}} \times \overline{\boldsymbol{b}^{*}}-\mathcal{E}^{*}+\overline{\boldsymbol{J}^{*}}, \quad \text { where } \quad \mathcal{E}^{*} \equiv \overline{\boldsymbol{u}^{* \prime} \times \boldsymbol{b}^{* \prime}}
$$

is the mean electromotive force (EMF), while its fluctuating part gives

$$
\boldsymbol{E}^{* \prime}=-\overline{\boldsymbol{u}^{*}} \times \boldsymbol{b}^{* \prime}-\boldsymbol{u}^{* \prime} \times \overline{\boldsymbol{b}^{*}}-\left(\boldsymbol{u}^{* \prime} \times \boldsymbol{b}^{* \prime}-\mathcal{E}^{*}\right)+\boldsymbol{J}^{* \prime} .
$$

The mean and fluctuating parts of the mass flux (2b) are simply

$$
\overline{\boldsymbol{m}^{*}}=\overline{\rho^{*}} \overline{\boldsymbol{u}^{*}}+\overline{\rho^{* \prime} \boldsymbol{u}^{* \prime}}, \quad \boldsymbol{m}^{* \prime}=\overline{\rho^{*}} \boldsymbol{u}^{* \prime}+\boldsymbol{u}^{* \prime} \overline{\rho^{*}}+\left(\rho^{* \prime} \boldsymbol{u}^{* \prime}-\overline{\rho^{* \prime} \boldsymbol{u}^{* \prime}}\right) .
$$

Writing $q^{*}=\overline{q^{*}}+q^{* \prime}$, the mean part of the heat conducting equation (3a) is

$$
\partial_{t} \overline{\theta^{*}}+\overline{u^{*}} \cdot \nabla \overline{\theta^{*}}=\kappa \Delta \overline{\theta^{*}}+\mathcal{H}^{*}+\overline{q^{*}}, \quad \text { where } \quad \mathcal{H}^{*} \equiv-\overline{\boldsymbol{u}^{* \prime} \cdot \nabla \theta^{*^{\prime}}},
$$

while its fluctuating part is

$$
\partial_{t} \theta^{* \prime}+\overline{\boldsymbol{u}^{*}} \cdot \boldsymbol{\nabla} \theta^{* \prime}+\boldsymbol{u}^{* \prime} \cdot \nabla \overline{\theta^{*}}+\left(\boldsymbol{u}^{* \prime} \cdot \nabla \theta^{* \prime}+\mathcal{H}^{*}\right)=\kappa \Delta \theta^{* \prime}+q^{* \prime} .
$$

HEL and its variants are motivated by flows which are dominated by mean fields such as $\overline{\boldsymbol{u}^{*}}, \overline{\boldsymbol{b}^{*}}$ and $\overline{\rho^{*}}, \overline{\theta^{*}}$ and are characterised by large magnetic Reynolds number $R m$ and large Péclet number $P e$ based on a typical mean-field length $\mathcal{L}$ and mean velocity $\mathcal{U}$ :

$$
R m=\mathcal{L} \mathcal{U} / \mathfrak{K} \gg 1, \quad P e=\mathcal{L} \mathcal{U} / \kappa \gg 1 \quad\left(\overline{\boldsymbol{u}^{*}}=\mathrm{O}(\mathcal{U})\right) .
$$

In the Braginsky $(1964 a, b)$ case of particular interest to us, the fluctuations are asymmetric wave perturbations to an axisymmetric mean state, which in his application are generally on the same length scale as the mean state. The central issue for all mean-field theories is how best to deal with these fluctuations. One method due to Frieman and Rotenberg (1960), appropriate to small amplitude disturbances, provides the key to addressing Braginsky's nearly axisymmetric dynamo. It builds on the following alternative representation of the fluctuating velocity in terms of a new fluctuating vector field (or effective-displacement) $\zeta$ :

$$
\boldsymbol{u}^{* \prime}(\boldsymbol{x}, t)=\partial_{t} \boldsymbol{\zeta}-\left[\boldsymbol{\zeta}, \overline{\boldsymbol{u}^{*}}\right], \quad \overline{\boldsymbol{\zeta}}=\mathbf{0}
$$

(see Soward and Roberts 2008, eqs. (3.32a,c)), where the antisymmetric bilinear operator $[\boldsymbol{\zeta}, \bullet]$ is defined by

$$
[\boldsymbol{\zeta}, \boldsymbol{u}] \equiv \boldsymbol{\zeta} \cdot \boldsymbol{\nabla} \boldsymbol{u}-\boldsymbol{u} \cdot \boldsymbol{\nabla} \boldsymbol{\zeta}=-\boldsymbol{\nabla} \times(\boldsymbol{\zeta} \times \boldsymbol{u})-(\boldsymbol{\nabla} \cdot \boldsymbol{\zeta}) \boldsymbol{u}+\boldsymbol{\zeta}(\boldsymbol{\nabla} \cdot \boldsymbol{u})
$$

satisfying $-[\boldsymbol{\zeta}, \boldsymbol{u}]=[\boldsymbol{u}, \boldsymbol{\zeta}]$. For later use, we also introduce

$$
\begin{aligned}
\llbracket \boldsymbol{\zeta}, \boldsymbol{m} \rrbracket & \equiv[\boldsymbol{\zeta}, \boldsymbol{m}]+(\boldsymbol{\nabla} \cdot \boldsymbol{\zeta}) \boldsymbol{m}=-\boldsymbol{\nabla} \times(\boldsymbol{\zeta} \times \boldsymbol{m})+\boldsymbol{\zeta}(\boldsymbol{\nabla} \cdot \boldsymbol{m}), \\
\{\boldsymbol{\zeta}, \boldsymbol{J}\} & \equiv \boldsymbol{\zeta} \cdot \boldsymbol{\nabla} \boldsymbol{J}+(\boldsymbol{\nabla} \boldsymbol{\zeta}) \cdot \boldsymbol{J}=-\boldsymbol{\zeta} \times(\boldsymbol{\nabla} \times \boldsymbol{J})+\boldsymbol{\nabla}(\boldsymbol{\zeta} \cdot \boldsymbol{J}),
\end{aligned}
$$

where in component form $((\boldsymbol{\nabla} \boldsymbol{\zeta}) \cdot \boldsymbol{J})_{i} \equiv\left(\nabla_{i} \zeta_{j}\right) J_{j}$. Each of (10a-c) are examples of Lie derivatives $\mathrm{L}_{\boldsymbol{\zeta}} \bullet$ (see $(99 \mathrm{c}-\mathrm{e})$, also Appendix A). In principle, given the velocity field $\boldsymbol{u}^{*}$ decomposed as in (4a) into its mean and fluctuating parts $\overline{\boldsymbol{u}^{*}}$ and $\boldsymbol{u}^{* \prime},(9 \mathrm{a})$ may be solved as an initial value problem to determine $\boldsymbol{\zeta}$ whose solution is rendered unique by demanding that $\boldsymbol{\zeta}=\mathbf{0}$ at the initial instant. Furthermore, in the special case of constant density fluids ( $\rho^{*}=\rho_{0}=$ constant) for which $\boldsymbol{\nabla} \cdot \overline{\boldsymbol{u}^{*}}=0$ and $\boldsymbol{\nabla} \cdot \boldsymbol{u}^{* \prime}=0$, the divergence of (9a) and use of (10a) determines $\left(\partial_{t}+\overline{\boldsymbol{u}^{*}} \cdot \boldsymbol{\nabla}\right)(\boldsymbol{\nabla} \cdot \boldsymbol{\zeta})=0$. Its solution subject to the initial condition $\boldsymbol{\nabla} \cdot \boldsymbol{\zeta}=0$ is

$$
\boldsymbol{\nabla} \cdot \boldsymbol{\zeta}=0, \quad \text { when } \quad \boldsymbol{\nabla} \cdot \boldsymbol{u}^{*}=0 .
$$

Under those combined solenoidal conditions the expression (9a) for $\boldsymbol{u}^{* \prime}$ reduces with the help of (10a) to

$$
\boldsymbol{u}^{* \prime}(\boldsymbol{x}, t)=\partial_{t} \boldsymbol{\zeta}+\boldsymbol{\nabla} \times\left(\boldsymbol{\zeta} \times \overline{\boldsymbol{u}^{*}}\right)
$$


In the remainder of this introduction, we explain how an asymptotic expansion, based on

$$
|\zeta| \ll \mathcal{L},
$$

can be developed within an Eulerian setting for large magnetic Reynolds and Péclet numbers $R m$ and $P e$ (see $(8 \mathrm{a}, \mathrm{b}))$. Though we take advantage of the effective-displacement $\zeta$ via the representation (9a) of the fluctuating velocity $\boldsymbol{u}^{* \prime}$, the analysis is cumbersome. Our relatively succinct summary in sections 1.2 and 1.3 hides the gruesome details, which are relegated to Appendices $\mathrm{C}$ and $\mathrm{D}$ respectively (some succour is provided by expressions for the Lie derivatives (10a-c) of products given in Appendix B). In section 1.4 we use the apparatus set up to recover Braginsky's (1964a, 1964b) formulation of his mean-field dynamo. The main purpose of our lengthy explanation is to emphasise that, though this Eulerian approach is natural, its implementation is far from obvious. We reiterate the final point made in our preamble that there is a much better way using Lagrangian ideas to attain the final Eulerian formulation, which is both more illuminating and amenable to generalisation.

In section 2, we introduce the Hybrid Euler-Lagrange method, which involves a small Lagrangian HEL-displacement $\boldsymbol{\xi}$. In section 3, we introduce yet another small ETHELdisplacement $\boldsymbol{\eta}$ which effects an Eulerian Transformed HEL leading to the Eulerian setting of this Introduction. At lowest order each of the three small displacement variables $\boldsymbol{\zeta}, \boldsymbol{\xi}$ and $\boldsymbol{\eta}$ are the same. Frieman and Rotenberg (1960) developed their method for the limiting case of perfect fluids for which the magnetic diffusivity $\mathfrak{K}$ and the thermal diffusivity $\kappa$ both vanish. Since this entire paper builds upon that limit, we review that limit in the following section 1.2 , where we find it convenient to introduce the symbol $\simeq$ also $\approx$ and $\approx$, which we define by their actions

$$
\simeq \bullet \mapsto=\bullet+\mathrm{O}\left(\eta^{2}\right), \quad \approx \bullet \mapsto=\bullet+\mathrm{O}\left(\eta^{3}\right), \quad \approx \bullet \mapsto=\bullet+\mathrm{O}\left(\eta^{4}\right)(13 \mathrm{a}-\mathrm{c})
$$

on some quantity $\bullet$, where $\mathrm{O}(\zeta)=\mathrm{O}(\xi)=\mathrm{O}(\eta)$ or $\boldsymbol{\zeta} \simeq \boldsymbol{\eta}, \boldsymbol{\xi} \simeq \boldsymbol{\eta}$ and e.g. $\boldsymbol{\zeta} \cdot(\boldsymbol{\zeta}-\boldsymbol{\eta}) \approx 0$, $|\boldsymbol{\zeta}-\boldsymbol{\eta}|^{2} \approx 0$. When the second large parameter $R m$ is introduced, we continue to use the notation (13a-c) to mean that they hold with all corrections dependent on $\mathrm{Rm}^{-1}$ neglected, i.e., $R m^{-1}=0$. In that spirit, later in section 3.3 , we introduce

$$
\doteq \bullet \quad \mapsto \quad \bullet+\mathrm{O}\left(R m^{-1} \eta^{2}\right)
$$

to mean exact equality, when $R m^{-1}=0$, but possesses error $\mathrm{O}\left(R m^{-1} \eta^{2}\right)$ at finite $R m$. However, in section 1.3 when we first consider the effects of finite diffusivity, we tighten up the notation $(13 \mathrm{a}, \mathrm{b})$ by defining

$$
\bumpeq \bullet \mapsto=\bullet+\mathrm{O}\left(\eta^{2}\right)+\mathrm{o}\left(R m^{-1} \eta\right), \quad \approx \bullet \mapsto=\bullet+\mathrm{O}\left(\eta^{3}\right)+\mathrm{o}\left(R m^{-1} \eta^{2}\right),
$$

for results correct to $\mathrm{O}\left(R m^{-1} \eta\right)$ and $\mathrm{O}\left(R m^{-1} \eta^{2}\right)$ respectively at finite $R m$. We use a similar notation to (14) and $(15 \mathrm{a}, \mathrm{b})$ for thermal corrections involving the small inverse Péclet number $P e^{-1}$. When, however, we consider the Braginsky dynamo in section 1.4, we adopt the relation $\eta=\mathrm{O}\left(R m^{-1 / 2}\right)$. Then our symbols $(13 \mathrm{a}-\mathrm{c}),(14)$ and $(15 \mathrm{a}, \mathrm{b})$ become ambiguous and so we use the symbol $\doteqdot$ to mean 'equal correct to lowest order with respect to all small parameters'. Very occasionally we take advantage of the 'catch-all' meaning of $\doteqdot$ in the context of $\doteqdot \bullet \mapsto=$ - $+\mathrm{O}(\eta)$ not encompassed by (13a-c), e.g., $\boldsymbol{u}^{*} \doteqdot \overline{\boldsymbol{u}^{*}}$. We outline the HEL based development of the remainder of the paper in section 1.5.

\section{$1.2 \quad$ Perfect fluids}

The Frieman and Rotenberg (1960) procedure is ideally suited to perfect fluids for which diffusion is ignored and there is no heat source. That means that we ignore the terms $\boldsymbol{J}^{*}$ in (1b), and $\kappa \Delta \theta^{*}$ and $q^{*}$ in (3a) as well as all their subsequent occurrences in (4) - (7). The essential assumption is that fluctuations are small $\mathrm{O}(\zeta)$ relative to their mean parts. By 
implication the quadratic products of fluctuations are even smaller $\mathrm{O}\left(\zeta^{2}\right)$. So to lowest order, we linearise the equations governing fluctuations at $\mathrm{O}(\zeta)$. Consistently, when dealing with the mean-field equations at $\mathrm{O}\left(\zeta^{2}\right)$, we evaluate the mean transport terms $\overline{\boldsymbol{u}^{* \prime} \times \boldsymbol{b}^{* \prime}}, \overline{\rho^{* \prime} \boldsymbol{u}^{* \prime}}$ and $\overline{\boldsymbol{u}^{* \prime} \cdot \boldsymbol{\nabla} \theta^{* \prime}}$ in the governing equations using the linearised results for the fluctuations. The approximation is sometimes referred to as the First Order Smoothing Approximation (FOSA); though its basis in turbulence theory does not necessarily rely on the small size of the unaveraged products of fluctuations but rather on the small size of their statistical average. Below we summarise the results whose derivation we outline in Appendix C.

Under the FOSA approximations the $\mathrm{O}(\zeta)$ fluctuations $\boldsymbol{b}^{* \prime}, \rho^{* \prime}$ and $\theta^{* \prime}$ are governed by

$$
\partial_{t} \boldsymbol{b}^{* \prime}=-\nabla \times \boldsymbol{E}^{* \prime}, \quad \boldsymbol{E}^{* \prime} \simeq-\overline{\boldsymbol{u}^{*}} \times \boldsymbol{b}^{* \prime}-\boldsymbol{u}^{* \prime} \times \overline{\boldsymbol{b}^{*}}, \quad \boldsymbol{\nabla} \cdot \boldsymbol{b}^{* \prime}=0 \quad(16 \mathrm{a}-\mathrm{c})
$$

$($ see $(1 \mathrm{a}, \mathrm{d})$ and $(5 \mathrm{c}))$

$$
\partial_{t} \rho^{* \prime}=-\nabla \cdot \boldsymbol{m}^{* \prime}, \quad \boldsymbol{m}^{* \prime} \simeq \overline{\rho^{*}} \boldsymbol{u}^{* \prime}+\boldsymbol{u}^{* \prime} \overline{\rho^{*}}
$$

(see $(2 \mathrm{a})$ and $(6 \mathrm{~b}))$ and

$$
\partial_{t} \theta^{* \prime}+\overline{\boldsymbol{u}^{*}} \cdot \nabla \theta^{* \prime}+\boldsymbol{u}^{* \prime} \cdot \nabla \overline{\theta^{*}} \simeq 0
$$

(see $(7 \mathrm{c})$ ) respectively, in which we have utilised the order equality symbol $\simeq$ (see (13a)). In Appendix C.1, we show that they have the particular solutions

$$
\boldsymbol{b}^{* \prime} \simeq \nabla \times\left(\boldsymbol{\zeta} \times \overline{\boldsymbol{b}^{*}}\right), \quad \quad \rho^{* \prime} \simeq-\nabla \cdot\left(\overline{\rho^{*}} \boldsymbol{\zeta}\right), \quad \theta^{* \prime} \simeq-\boldsymbol{\zeta} \cdot \nabla \overline{\theta^{*}},
$$

which satisfy the initial conditions $\boldsymbol{b}^{* \prime}=\mathbf{0}, \rho^{* \prime}=0$ and $\theta^{* \prime}=0$ when $\boldsymbol{\zeta}=\mathbf{0}$ at $t=0$, with

$$
\begin{aligned}
\boldsymbol{E}^{* \prime} & \simeq-\partial_{t}\left(\boldsymbol{\zeta} \times \overline{\boldsymbol{b}^{*}}\right)-\nabla\left(\boldsymbol{\zeta} \cdot \overline{\boldsymbol{E}^{*}}\right), & \overline{\boldsymbol{E}^{*}} & \simeq-\overline{\boldsymbol{u}^{*}} \times \overline{\boldsymbol{b}^{*}}, \\
\boldsymbol{m}^{* \prime} & \simeq \partial_{t}\left(\overline{\rho^{*} \boldsymbol{\zeta}}\right)+\nabla \times\left(\boldsymbol{\zeta} \times \overline{\boldsymbol{m}^{*}}\right), & \overline{\boldsymbol{m}^{*}} & \simeq \overline{\rho^{*}} \overline{\boldsymbol{u}^{*}} .
\end{aligned}
$$

We remark that in the constant density fluid case, (19a) may be expressed with the help of the identity (10a) and (22c) in the alternative form

$$
\boldsymbol{b}^{* \prime} \simeq-\left[\boldsymbol{\zeta}, \overline{\boldsymbol{b}^{*}}\right], \quad \text { when } \quad \boldsymbol{\nabla} \cdot \boldsymbol{\zeta}=0 .
$$

The above explicit solutions for the fluctuations provide an excellent starting point for the continuation of our expansion procedures to higher orders of approximation.

The mean quantities $\overline{\boldsymbol{b}^{*}}, \overline{\rho^{*}}$ and $\overline{\theta^{*}}$ are governed by

$$
\partial_{t} \overline{\boldsymbol{b}^{*}}=-\nabla \times \overline{\boldsymbol{E}^{*}}, \quad \overline{\boldsymbol{E}^{*}}=-\overline{\boldsymbol{u}^{*}} \times \overline{\boldsymbol{b}^{*}}-\overline{\boldsymbol{u}^{* \prime} \times \boldsymbol{b}^{* \prime}}, \quad \boldsymbol{\nabla} \cdot \overline{\boldsymbol{b}^{*}}=0
$$

(see $(1 \mathrm{a}, \mathrm{d})$ and $(5 \mathrm{a}, \mathrm{b}))$,

$$
\partial_{t} \overline{\rho^{*}}=-\nabla \cdot \overline{\boldsymbol{m}^{*}}, \quad \overline{\boldsymbol{m}^{*}}=\overline{\rho^{*}} \overline{\boldsymbol{u}^{*}}+\overline{\rho^{* \prime} \boldsymbol{u}^{* \prime}}
$$

(see $(2 \mathrm{a})$ and $(6 \mathrm{a}))$ and

$$
\partial_{t} \overline{\theta^{*}}+\overline{u^{*}} \cdot \nabla \overline{\theta^{*}}=-\overline{u^{* \prime} \cdot \nabla \theta^{* \prime}}
$$

(see (7a,b)) respectively. Remarkably, correct to $\mathrm{O}\left(\zeta^{2}\right)$, the mean transport terms $\overline{\boldsymbol{u}^{* \prime} \times \boldsymbol{b}^{* \prime}}$, $\overline{\rho^{* \prime} \boldsymbol{u}^{* \prime}}$ and $\overline{\boldsymbol{u}^{* \prime} \cdot \boldsymbol{\nabla} \theta^{* \prime}}$, that are generally troublesome in mean-field theories, can be absorbed into the products of means by the following judicious choice of new mean variables

$$
\begin{array}{rlrl}
\boldsymbol{u}^{\dagger} & \approx \overline{\boldsymbol{u}^{*}}+\frac{1}{2} \overline{\left[\boldsymbol{\zeta}, \boldsymbol{u}^{* \prime}\right]}, & & \boldsymbol{b}^{\dagger} \approx \overline{\boldsymbol{b}^{*}}-\frac{1}{2} \boldsymbol{\nabla} \times\left(\overline{\boldsymbol{\zeta} \times \boldsymbol{b}^{* \prime}}\right), \\
\rho^{\dagger} \approx \overline{\rho^{*}}+\frac{1}{2} \boldsymbol{\nabla} \cdot\left(\overline{\rho^{* \prime} \boldsymbol{\zeta}}\right), & & \theta^{\dagger} \approx \overline{\theta^{*}}+\frac{1}{2} \overline{\boldsymbol{\zeta} \cdot \boldsymbol{\nabla} \theta^{* \prime}}
\end{array}
$$

(see $(13 \mathrm{~b})$ for the definition of $\approx$ ), where $\boldsymbol{u}^{* \prime}$ is given by $(9 \mathrm{a})$, while $\boldsymbol{b}^{* \prime}, \rho^{* \prime}$ and $\theta^{* \prime}$ are given correct to $\mathrm{O}(\zeta)$ by $(19 \mathrm{a}-\mathrm{c})$. The new $\dagger$ 'd variables have the initial value properties $\boldsymbol{u}^{\dagger}=\boldsymbol{u}^{*}$, 
$\boldsymbol{b}^{\dagger}=\boldsymbol{b}^{*}, \rho^{\dagger}=\rho^{*}$ and $\theta^{\dagger}=\theta^{*}$ when $\boldsymbol{\zeta}=\mathbf{0}$ at $t=0$. They are supplemented by

$$
\begin{aligned}
\boldsymbol{E}^{\dagger} & \approx \overline{\boldsymbol{E}^{*}}+\frac{1}{2} \partial_{t}\left(\overline{\boldsymbol{\zeta} \times \boldsymbol{b}^{* \prime}}\right)+\frac{1}{2} \boldsymbol{\nabla}\left(\overline{\boldsymbol{\zeta} \cdot \boldsymbol{E}^{* \prime}}\right), \\
\boldsymbol{m}^{\dagger} & \approx \overline{\boldsymbol{m}^{*}}-\frac{1}{2} \partial_{t}\left(\overline{\rho^{* \prime} \boldsymbol{\zeta}}\right)-\frac{1}{2} \boldsymbol{\nabla} \times\left(\overline{\boldsymbol{\zeta} \times \boldsymbol{m}^{* \prime}}\right),
\end{aligned}
$$

where $\boldsymbol{E}^{* \prime}$ and $\boldsymbol{m}^{* \prime}$ are given correct to $\mathrm{O}(\zeta)$ by (20a-d). Indeed, we show in Appendix C.2 that $\boldsymbol{u}^{\dagger}, \boldsymbol{b}^{\dagger}, \rho^{\dagger}, \theta^{\dagger}$ and $\boldsymbol{E}^{\dagger}, \boldsymbol{m}^{\dagger}$ defined by (25a-d) and (26a,b) satisfy

$$
\begin{aligned}
& \partial_{t} \boldsymbol{b}^{\dagger}=-\boldsymbol{\nabla} \times \boldsymbol{E}^{\dagger}, \quad \boldsymbol{E}^{\dagger}=-\boldsymbol{u}^{\dagger} \times \boldsymbol{b}^{\dagger}, \quad \boldsymbol{\nabla} \cdot \boldsymbol{b}^{\dagger}=0, \\
& \partial_{t} \rho^{\dagger}=-\nabla \cdot \boldsymbol{m}^{\dagger}, \quad \boldsymbol{m}^{\dagger}=\rho^{\dagger} \boldsymbol{u}^{\dagger}, \\
& \partial_{t} \theta^{\dagger}+\boldsymbol{u}^{\dagger} \cdot \nabla \theta^{\dagger}=0
\end{aligned}
$$

correct to $\mathrm{O}\left(\zeta^{2}\right)$. Evidently there is some freedom about which relations we regard as approximate and which we regard as exact. Later we will want to regard the †'d variables as HEL-variables for which the relations (25a-d), (26a,b) are approximate, while the equations (27a-c), (28a,b) and (29) are exact. This consideration guides our definitions in the next subsection also.

We remark that in the constant density case for which $\boldsymbol{\nabla} \cdot \boldsymbol{\zeta}=0$ and $\boldsymbol{\nabla} \cdot \boldsymbol{u}^{*}=0$ (see $(11 \mathrm{a}, \mathrm{b})$ ), the Stokes drift velocity $\boldsymbol{u}^{\dagger}-\overline{\boldsymbol{u}^{*}} \approx \frac{1}{2} \overline{\left[\boldsymbol{\zeta}, \boldsymbol{u}^{* \prime}\right]}$ defined by (25a) is solenoidal too. Then the magnetic induction equation derived from (27a-c) is simply

$$
\partial_{t} \boldsymbol{b}^{\dagger}+\left[\boldsymbol{u}^{\dagger}, \boldsymbol{b}^{\dagger}\right]=0 \quad \text { with } \quad \boldsymbol{\nabla} \cdot \boldsymbol{u}^{\dagger}=0, \quad \boldsymbol{\nabla} \cdot \boldsymbol{b}^{\dagger}=0 .
$$

Vladimirov (2012) considered the case of high frequency waves, for which $\boldsymbol{u}^{* \prime} \doteqdot \partial_{t} \boldsymbol{\zeta}$ (see (9a) with $\left|\partial_{t} \boldsymbol{\zeta}\right| \gg\left|\left[\boldsymbol{\zeta}, \overline{\boldsymbol{u}^{*}}\right]\right|$ ). In that limit, $\boldsymbol{u}^{\dagger}-\overline{\boldsymbol{u}^{*}} \doteqdot \frac{1}{2} \overline{\left[\boldsymbol{\zeta}, \partial_{t} \boldsymbol{\zeta}\right]}$ (his eq. (4.8), but note that his [, ] is defined with the opposite sign to ours), while $\boldsymbol{b}^{\dagger} \doteqdot \overline{\boldsymbol{b}}^{*}$ in (30a) (his eq. (4.9b)). Vladimirov's methods relate tenuously to those, which we employ in Appendix C, in as much as he too appeals to properties of Lie derivatives.

\subsection{Small but finite diffusivity}

The diffusion processes, that operate in real fluids, modify the expansions of section 1.2 with the exception of density $\rho$, for which the mass continuity equation (2a) continues to hold. So when the fluid has finite electrical conductivity $\sigma$ and thermal diffusivity $\kappa$ the small terms $\boldsymbol{J}^{*}$ in (1b) and $\kappa \Delta \theta^{*}, q^{*}$ in (3a) must now be retained; their importance is measured by the inverses of the large magnetic Reynolds and Péclet numbers $R m$ and $P e$ (see $(8 \mathrm{a}, \mathrm{b})$ ). Here we extend the results reported in section 1.2 to include corrections linear in $R m^{-1}$ and $P e^{-1}$. To that end we need to define $\mathrm{O}\left(R m^{-1}\right)$ and $\mathrm{O}\left(P e^{-1}\right)$ corrections to $\boldsymbol{b}^{\dagger}$ and $\theta^{\dagger}$, when they appear in the advective terms appearing in section 1.2 , but only retain the $\mathrm{O}(1)$ contributions (relative to the $R m^{-1}$ and $P e^{-1}$ expansion parameters) in the small diffusion terms $\boldsymbol{J}^{*}$ and $\kappa \Delta \theta^{*}$ in (1b) and (3a). We continue to make the FOSA assumption. Here, we only summarise the results but provide their derivations in Appendix D.

As in section 1.2, we begin by considering the $\mathrm{O}(\zeta)$ fluctuations. Instead of $(16 \mathrm{~b})$ we approximate $(5 \mathrm{c})$ by

$$
\boldsymbol{E}^{* \prime} \bumpeq-\overline{\boldsymbol{u}^{*}} \times \boldsymbol{b}^{* \prime}-\boldsymbol{u}^{* \prime} \times \overline{\boldsymbol{b}^{*}}+\boldsymbol{J}^{* \prime}, \quad \text { where } \quad \boldsymbol{J}^{* \prime}=\mathfrak{K} \boldsymbol{\nabla} \times \boldsymbol{b}^{* \prime}
$$

(see $(1 \mathrm{~g})$ ), while in place of (18) we approximate (7c) by

$$
\partial_{t} \theta^{* \prime}+\overline{\boldsymbol{u}^{*}} \cdot \nabla \theta^{* \prime}+\boldsymbol{u}^{* \prime} \cdot \nabla \overline{\theta^{*}} \bumpeq \kappa \Delta \theta^{* \prime}+q^{* \prime},
$$

where we assume that $q^{* \prime}=\mathrm{O}\left(\left|\kappa \Delta \theta^{* \prime}\right|\right)$. Accordingly, the expressions (19a,c) for $\boldsymbol{b}^{* \prime}$ and $\theta^{* \prime}$ possess corrections

$$
\boldsymbol{b}^{\dagger \prime} \bumpeq \boldsymbol{b}^{* \prime}-\nabla \times\left(\boldsymbol{\zeta} \times \overline{\boldsymbol{b}^{*}}\right), \quad \theta^{\dagger \prime} \bumpeq \theta^{* \prime}+\boldsymbol{\zeta} \cdot \nabla \overline{\theta^{*}},
$$


smaller by factors $R m^{-1}$ and $P e^{-1}$ respectively (whence our use of $\bumpeq$, see (15a)) stemming from the diffusion terms $\boldsymbol{J}^{* \prime}$ in (31a) and $\kappa \Delta \theta^{* \prime}$ in (32). We write the corresponding correction for $\boldsymbol{E}^{* \prime}$ in the form

$$
\boldsymbol{E}^{\dagger \prime} \bumpeq \boldsymbol{E}^{* \prime}+\partial_{t}\left(\boldsymbol{\zeta} \times \overline{\boldsymbol{b}^{*}}\right)+\nabla\left(\boldsymbol{\zeta} \cdot \overline{\boldsymbol{E}^{*}}\right)
$$

in which

$$
\overline{\boldsymbol{E}^{*}} \bumpeq-\overline{\boldsymbol{u}^{*}} \times \overline{\boldsymbol{b}^{*}}+\overline{\boldsymbol{J}^{*}}, \quad \text { where } \quad \overline{\boldsymbol{J}^{*}}=\mathfrak{K} \boldsymbol{\nabla} \times \overline{\boldsymbol{b}^{*}}
$$

(see $(1 \mathrm{~b}, \mathrm{~g}))$. In Appendix D.1 we show that $\boldsymbol{b}^{\dagger \prime}$ satisfies

$$
\partial_{t} \boldsymbol{b}^{\dagger \prime}=-\boldsymbol{\nabla} \times \boldsymbol{E}^{\dagger \prime}, \quad \boldsymbol{E}^{\dagger \prime} \bumpeq-\overline{\boldsymbol{u}^{*}} \times \boldsymbol{b}^{\dagger \prime}+\left(\boldsymbol{J}^{* \prime}+\left\{\boldsymbol{\zeta}, \overline{\boldsymbol{J}^{*}}\right\}\right)
$$

with $\boldsymbol{\nabla} \cdot \boldsymbol{b}^{\dagger \prime}=0$, where the bilinear operator $\{\boldsymbol{\zeta}, \bullet\}$ is defined by (10c), while $\theta^{\dagger \prime}$ satisfies

$$
\partial \theta^{\dagger \prime}+\overline{\boldsymbol{u}^{*}} \cdot \boldsymbol{\nabla} \theta^{\dagger \prime} \bumpeq \kappa\left(\Delta \theta^{* \prime}+\boldsymbol{\zeta} \cdot \boldsymbol{\nabla}\left(\Delta \overline{\theta^{*}}\right)\right)+q^{\dagger \prime}, \quad q^{\dagger \prime} \bumpeq q^{* \prime}+\boldsymbol{\zeta} \cdot \boldsymbol{\nabla} \overline{q^{*}} .
$$

Our strategy is to construct $\dagger$ 'd variables as in section 1.2. We emphasise that we continue to use the variables $\boldsymbol{u}^{\dagger}, \rho^{\dagger}$ and $\boldsymbol{m}^{\dagger}$ defined by $(25 \mathrm{a}, \mathrm{c})$ and $(26 \mathrm{~b})$. As there, we demand that

$$
\boldsymbol{u}^{\dagger}=\overline{\boldsymbol{u}^{\dagger}}, \quad \quad \rho^{\dagger}=\overline{\rho^{\dagger}}, \quad \boldsymbol{m}^{\dagger}=\overline{\boldsymbol{m}^{\dagger}}
$$

have no fluctuating parts and that they satisfy the mass continuity equations (28a,b). Nevertheless, our magnetic and thermal †'d variables have both mean and very small fluctuating parts:

$$
\boldsymbol{b}^{\dagger}=\overline{\boldsymbol{b}^{\dagger}}+\boldsymbol{b}^{\dagger \prime}, \quad \boldsymbol{E}^{\dagger}=\overline{\boldsymbol{E}^{\dagger}}+\boldsymbol{E}^{\dagger \prime}, \quad \theta^{\dagger}=\overline{\theta^{\dagger}}+\theta^{\dagger \prime},
$$

in which $\boldsymbol{b}^{\dagger \prime}=\mathrm{O}\left(R m^{-1} \boldsymbol{b}^{* \prime}\right), \boldsymbol{E}^{\dagger \prime}=\mathrm{O}\left(R m^{-1} \boldsymbol{E}^{* \prime}\right)$ and $\theta^{\dagger \prime}=\mathrm{O}\left(P e^{-1} \theta^{* \prime}\right)$ all vanish in the perfect fluid limit.

Surprisingly, the appropriate choice of the forms (25b), (26a) and (25d) for $\boldsymbol{b}^{\dagger}, \boldsymbol{E}^{\dagger}$ and $\theta^{\dagger}$ respectively, correct to $\mathrm{O}\left(\zeta^{2}\right)$, continues to apply to $\overline{\boldsymbol{b}^{\dagger}}, \overline{\boldsymbol{E}^{\dagger}}$ and $\overline{\theta^{\dagger}}$ but with one minor structural change, namely $\boldsymbol{b}^{* \prime}, \boldsymbol{E}^{* \prime}$ and $\theta^{* \prime}$ in (25b), (26a) and (25d) are replaced by the variables

$$
\widetilde{\boldsymbol{b}}^{\dagger \prime}=\boldsymbol{b}^{* \prime}+\boldsymbol{b}^{\dagger \prime}, \quad \widetilde{\boldsymbol{E}}^{\dagger \prime}=\boldsymbol{E}^{* \prime}+\boldsymbol{E}^{\dagger \prime}, \quad \widetilde{\theta}^{\dagger \prime}=\theta^{* \prime}+\theta^{\dagger \prime},
$$

which we emphasise are distinguished by the presence of the tilde. The analysis of Appendix D.2 shows that the resulting forms

$$
\begin{aligned}
& \overline{\boldsymbol{b}^{\dagger}} \approx \overline{\boldsymbol{b}^{*}}-\frac{1}{2} \boldsymbol{\nabla} \times\left(\overline{\boldsymbol{\zeta} \times \widetilde{\boldsymbol{b}}^{\dagger \prime}}\right), \quad \overline{\boldsymbol{E}^{\dagger}} \approx \overline{\boldsymbol{E}^{*}}+\frac{1}{2} \partial_{t}\left(\overline{\boldsymbol{\boldsymbol { \zeta } \times \widetilde { \boldsymbol { b } } ^ { \dagger \prime }}}\right)+\frac{1}{2} \boldsymbol{\nabla}\left(\overline{\boldsymbol{\zeta} \cdot \widetilde{\boldsymbol{E}}^{\dagger \prime}}\right), \\
& \overline{\theta^{\dagger}} \approx \overline{\theta^{*}}+\frac{1}{2} \overline{\boldsymbol{\zeta} \cdot \boldsymbol{\nabla} \widetilde{\theta}^{\dagger \prime}}, \quad \overline{q^{\dagger}} \approx \overline{q^{*}}+\frac{1}{2} \overline{\boldsymbol{\zeta} \cdot \boldsymbol{\nabla} \widetilde{q}^{\dagger \prime}}, \quad \widetilde{q}^{\dagger \prime}=q^{* \prime}+q^{\dagger \prime}
\end{aligned}
$$

(see $(15 b)$ for the definition of $\approx$ ) satisfy

$$
\partial_{t} \overline{\boldsymbol{b}^{\dagger}}=-\boldsymbol{\nabla} \times \overline{\boldsymbol{E}^{\dagger}}, \quad \overline{\boldsymbol{E}^{\dagger}} \approx-\boldsymbol{u}^{\dagger} \times \overline{\boldsymbol{b}^{\dagger}}+\overline{\boldsymbol{J}^{*}}+\overline{\left\{\boldsymbol{\zeta}, \boldsymbol{J}^{* \prime}\right\}}+\frac{1}{2} \overline{\left\{\boldsymbol{\zeta},\left\{\boldsymbol{\zeta}, \overline{\boldsymbol{J}^{*}}\right\}\right\}},
$$

with $\nabla \cdot \overline{b^{\dagger}}=0$ and

$$
\left.\partial_{t} \overline{\theta^{\dagger}}+\boldsymbol{u}^{\dagger} \cdot \boldsymbol{\nabla} \overline{\theta^{\dagger}} \approx \kappa\left(\Delta \overline{\theta^{*}}+\overline{\boldsymbol{\zeta} \cdot \boldsymbol{\nabla}\left(\Delta \theta^{* \prime}+\frac{1}{2} \boldsymbol{\zeta} \cdot \boldsymbol{\nabla}\left(\Delta \overline{\theta^{*}}\right)\right.}\right)\right)+\overline{q^{\dagger}},
$$

correct to $\mathrm{O}\left(\zeta^{2}\right)$, where we assume that $\overline{q^{*}}=\mathrm{O}\left(\left|\kappa \Delta \overline{\theta^{*}}\right|\right)$. Whereas, our definitions (25a,c),

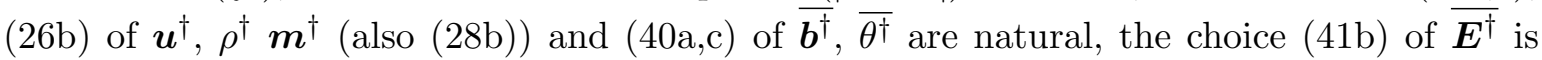
$a d h o c$ and only unique up to an additive gradient as indicated by the fact that only its curl is involved in the governing equation (41a). Our explicit choice that builds on our definition (34a) of $\boldsymbol{E}^{\dagger \prime}$ is motivated by our HEL-development in the later sections (but particularly the ETHEL-representation (111c,d) below). Following Braginsky (1964a), we will refer to the 
mean quantities $\boldsymbol{u}^{\dagger}, \overline{\boldsymbol{b}^{\dagger}}, \rho^{\dagger}, \overline{\theta^{\dagger}}, \overline{\boldsymbol{E}^{\dagger}}$ and $\boldsymbol{m}^{\dagger}$ as effective-variables, and whence our terminology effective-displacement for $\zeta$. The ultimate aim of our effective-variable apparatus is to develop the mean-field equations for $\overline{\boldsymbol{b}^{\dagger}}$ and $\overline{\theta^{\dagger}}$, which we summarise as follows:

- Effective magnetic field, $\overline{\boldsymbol{b}^{\dagger}}$. From $(41 \mathrm{a}, \mathrm{b})$, we may write

$$
\partial_{t} \overline{\boldsymbol{b}^{\dagger}}=\nabla \times\left(\boldsymbol{u}^{\dagger} \times \overline{\boldsymbol{b}^{\dagger}}+\mathcal{E}^{\dagger}\right)+\mathfrak{K} \Delta \overline{\boldsymbol{b}^{\dagger}},
$$

where the effective EMF $\mathcal{E}^{\dagger}$ is related to $\boldsymbol{E}^{\dagger}$ by

$$
\mathfrak{K}^{-1} \mathcal{E}^{\dagger} \equiv-\mathbf{J}^{\dagger}=-\mathfrak{K}^{-1}\left(\overline{\boldsymbol{E}^{\dagger}}+\boldsymbol{u}^{\dagger} \times \overline{\boldsymbol{b}^{\dagger}}\right)+\nabla \times \overline{\boldsymbol{b}^{\dagger}} .
$$

Here we introduce $\mathfrak{K} \mathbf{J}^{\dagger} \equiv-\mathcal{E}^{\dagger}$, because $\mathcal{E}^{\dagger}$ has closer links with electric current than the traditional EMF, as $(43 \mathrm{c}, \mathrm{d})$ below show. On recalling that $\mathfrak{K}^{-1} \overline{\boldsymbol{J}^{*}}=\boldsymbol{\nabla} \times \overline{\boldsymbol{b}^{*}}$, in which $\overline{\boldsymbol{b}^{*}} \approx \overline{\boldsymbol{b}^{\dagger}}+\frac{1}{2} \boldsymbol{\nabla} \times\left(\overline{\boldsymbol{\zeta} \times \boldsymbol{b}^{* \prime}}\right)$ (see $(25 \mathrm{~b})$ ), and $\mathfrak{K}^{-1} \boldsymbol{J}^{* \prime}=\boldsymbol{\nabla} \times \boldsymbol{b}^{* \prime}$ (see $(31 \mathrm{~b})$ ), substitution of $\overline{\boldsymbol{E}^{\dagger}}$ given by $(41 \mathrm{~b})$ into $(43 \mathrm{~b})$ leads to

$$
\begin{aligned}
& -\mathbf{J}^{\dagger} \approx \frac{1}{2} \Delta\left(\overline{\boldsymbol{\zeta} \times \boldsymbol{b}^{* \prime}}\right)-\overline{\boldsymbol{\zeta} \times \Delta \boldsymbol{b}^{* \prime}}+\frac{1}{2} \overline{\boldsymbol{\zeta} \times\left(\boldsymbol{\nabla} \times\left(\boldsymbol{\zeta} \times \Delta \overline{\boldsymbol{b}^{*}}\right)\right)} \\
& -\nabla\left(\frac{1}{2} \boldsymbol{\nabla} \cdot\left(\overline{\boldsymbol{\zeta} \times \boldsymbol{b}^{* \prime}}\right)+\overline{\boldsymbol{\zeta} \cdot\left(\boldsymbol{\nabla} \times \boldsymbol{b}^{* \prime}\right)}+\frac{1}{2} \overline{\boldsymbol{\zeta} \cdot \boldsymbol{\nabla}\left(\boldsymbol{\zeta} \cdot\left(\boldsymbol{\nabla} \times \overline{\boldsymbol{b}^{*}}\right)\right)}\right),
\end{aligned}
$$

in which the leading order approximation $\boldsymbol{b}^{* \prime} \simeq \boldsymbol{\nabla} \times\left(\boldsymbol{\zeta} \times \overline{\boldsymbol{b}^{*}}\right)$ (see (19a)) provides sufficient accuracy for the order needed. Of course, the gradient contribution in the second line of (43c) disappears in the mean effective magnetic induction equation (43a), as its curl vanishes. An interesting feature of (43c) is that, although it appears to involve the second and third derivatives of $\overline{\boldsymbol{b}^{*}}$, all these terms cancel and we are left with the structure

$$
-\mathbf{J}^{\dagger} \approx \overline{\boldsymbol{\alpha}^{\dagger}} \cdot \overline{\boldsymbol{b}^{\dagger}}-\left(\left(\overline{\boldsymbol{g}^{\ddagger}}-\mathbf{I}\right) \cdot \nabla\right) \times \overline{\boldsymbol{b}^{\dagger}},
$$

where $\overline{\boldsymbol{b}^{\dagger}} \simeq \overline{\boldsymbol{b}^{*}},\left(\left(\left(\overline{\boldsymbol{g}^{\ddagger}}-\mathbf{I}\right) \cdot \boldsymbol{\nabla}\right) \times \overline{\boldsymbol{b}^{\dagger}}\right)_{i} \equiv \epsilon_{i j k}\left(\overline{g_{j l}^{\ddagger}}-\delta_{j l}\right) \nabla_{l} \overline{b_{k}^{\dagger}}$ and

$$
\overline{\boldsymbol{g}^{\ddagger}}-\mathbf{I} \approx-\frac{1}{2} \overline{(\boldsymbol{\zeta} \cdot \boldsymbol{\nabla}) \mathbf{e}^{\ddagger}}+\frac{1}{2} \overline{\mathbf{e}^{\ddagger} \cdot(\boldsymbol{\nabla} \boldsymbol{\zeta})}+\frac{1}{2} \overline{(\boldsymbol{\nabla} \boldsymbol{\zeta})^{\mathrm{T}} \cdot \mathbf{e}^{\ddagger}}, \quad \mathbf{e}^{\ddagger} \simeq(\boldsymbol{\nabla} \boldsymbol{\zeta})+(\boldsymbol{\nabla} \boldsymbol{\zeta})^{\mathrm{T}} .
$$

The superscript ${ }^{\mathrm{T}}$ denotes the transpose $(\boldsymbol{\nabla} \boldsymbol{\zeta})_{i j}^{\mathrm{T}}=\nabla_{j} \zeta_{i}$, I is the identity matrix $\delta_{i j}$ and $\mathrm{e}^{\ddagger} i j=\nabla_{i} \zeta_{j}+\nabla_{j} \zeta_{i}$. The expression for the matrix $\overline{\boldsymbol{\alpha}^{\dagger}}$ is too lengthy to be reproduced here but is obtained trivially from the formulae (122a-d) for $\overline{\boldsymbol{\alpha}}$ on replacing $\boldsymbol{\eta}$ by $\boldsymbol{\zeta}$.

- Effective temperature, $\overline{\theta^{\dagger}}$. We may rewrite $(42)$ in the form

$$
\partial_{t} \overline{\theta^{\dagger}}+\boldsymbol{u}^{\dagger} \cdot \nabla \overline{\theta^{\dagger}}=\mathcal{H}^{\dagger}+\kappa \Delta \overline{\theta^{\dagger}}+\overline{q^{\dagger}}
$$

where, on recalling that $\Delta \overline{\theta^{*}} \approx \Delta \overline{\theta^{\dagger}}-\frac{1}{2} \Delta\left(\overline{\boldsymbol{\zeta} \cdot \boldsymbol{\nabla} \theta^{* \prime}}\right)$ (see $(25 \mathrm{~d})$ ),

$$
\kappa^{-1} \mathcal{H}^{\dagger} \equiv \mathrm{H}^{\dagger} \approx \overline{\boldsymbol{\zeta} \cdot \boldsymbol{\nabla}\left(\frac{1}{2} \boldsymbol{\zeta} \cdot \boldsymbol{\nabla}\left(\Delta \overline{\theta^{*}}\right)+\left(\Delta \theta^{* \prime}\right)\right)}-\frac{1}{2} \Delta \overline{\left(\overline{\boldsymbol{\zeta} \cdot \boldsymbol{\nabla} \theta^{* \prime}}\right)}
$$

with $\theta^{* \prime} \simeq-\boldsymbol{\zeta} \cdot \boldsymbol{\nabla} \overline{\theta^{*}}$ (see (19c)). Just like our introduction of $\mathfrak{K} \mathbf{J}^{\dagger} \equiv-\mathcal{E}^{\dagger}$ in (43b) above, we have here defined $\kappa \mathrm{H}^{\dagger} \equiv \mathcal{H}^{\dagger}$, because $\mathcal{H}^{\dagger}$ has closer links with thermal diffusion than heat transport. Furthermore, like the expression (43c) for $\mathbf{J}^{\dagger}$, the formula (45b) for $\mathrm{H}^{\dagger}$ involves second and third derivatives of $\nabla \overline{\theta^{*}}$, which again cancel leaving

$$
\mathrm{H}^{\dagger} \approx\left(\frac{1}{2} \boldsymbol{\nabla}(\overline{\boldsymbol{\zeta} \cdot \boldsymbol{\nabla}(\boldsymbol{\nabla} \cdot \boldsymbol{\zeta})})-\overline{\mathbf{e}^{\ddagger} \cdot \boldsymbol{\nabla}(\boldsymbol{\nabla} \cdot \boldsymbol{\zeta})}\right) \cdot \boldsymbol{\nabla} \overline{\theta^{*}}+\boldsymbol{\nabla} \cdot\left(\left(\overline{\boldsymbol{g}^{\ddagger}}-\mathbf{I}\right) \cdot \boldsymbol{\nabla} \overline{\theta^{*}}\right) .
$$

For the special case $\boldsymbol{\nabla} \cdot \boldsymbol{\zeta}=0$, the first term proportional to $\boldsymbol{\nabla} \overline{\theta^{*}}$ vanishes. 


\subsection{The Braginsky dynamo}

The mean magnetic induction equation in the effective-variable form (43a) together with the effective EMF (43b,c) lie at the heart of Braginsky's (1964a, 1964b) analysis of his mean-field dynamo. It is described relative to cylindrical polar coordinates $(s, \phi, z)$, for which we express vectors such as $\zeta$ and gradients by

$$
\boldsymbol{\zeta}=\left(\zeta_{s}, \zeta_{\phi}, \zeta_{z}\right), \quad \nabla \equiv\left(\frac{\partial}{\partial s}, \frac{1}{s} \frac{\partial}{\partial \phi}, \frac{\partial}{\partial z}\right)
$$

We use the abbreviations $\partial_{s} \equiv \partial / \partial s, \partial_{\phi} \equiv \partial / \partial \phi, \partial_{z} \equiv \partial / \partial z$, where $\partial_{\phi}$ does not differentiate unit vectors. Here, we depart from the notation of Braginsky, who employed $\partial_{1 \phi}$ when he held the unit vectors fixed. If we distinguish $\partial \boldsymbol{\zeta} / \partial \phi$ from $\partial_{\phi} \boldsymbol{\zeta}$, using the former to mean that the unit vectors are differentiated, we have

$$
\partial \boldsymbol{\zeta} / \partial \phi=\partial_{\phi} \boldsymbol{\zeta}+\widehat{\boldsymbol{z}} \times \boldsymbol{\zeta}, \quad \text { where } \quad \partial_{\phi} \boldsymbol{\zeta} \equiv\left(\partial_{\phi} \zeta_{s}, \partial_{\phi} \zeta_{\phi}, \partial_{\phi} \zeta_{z}\right)
$$

with, for example, the consequence

$$
\nabla \times(s \zeta \times \widehat{\phi})=\partial_{\phi} \zeta
$$

The mean state is axisymmetric and fluctuations have vanishing $\phi$-averages in a sense that we make precise in section 5. Attention is restricted to fluid with constant density $\rho^{*}=\rho_{0}$, so that the velocity $\boldsymbol{u}^{*}$ is solenoidal $\boldsymbol{\nabla} \cdot \boldsymbol{u}^{*}=0$ to which we add the condition $\boldsymbol{\nabla} \cdot \boldsymbol{\zeta}=0$ (see $(11 \mathrm{a}, \mathrm{b}))$. It is assumed that the flow velocity and magnetic field

$$
\begin{aligned}
\boldsymbol{u}^{*} & =\overline{u_{\phi}^{*}} \widehat{\boldsymbol{\phi}}+\boldsymbol{u}^{* \prime}+\overline{\boldsymbol{u}_{\mathrm{m}}^{*}}, & \overline{\boldsymbol{u}_{\mathrm{m}}^{*}} & =\nabla \times\left(\psi^{*} \widehat{\boldsymbol{\phi}}\right), \\
\boldsymbol{b}^{*} & =\overline{b_{\phi}^{*}} \widehat{\boldsymbol{\phi}}+\boldsymbol{b}^{* \prime}+\overline{\boldsymbol{b}_{\mathrm{m}}^{*}}, & \overline{\boldsymbol{b}_{\mathrm{m}}^{*}} & =\nabla \times\left(a^{*} \widehat{\boldsymbol{\phi}}\right)
\end{aligned}
$$

are dominated by their azimuthal components $\overline{u_{\phi}^{*}}$ and $\overline{b_{\phi}^{*}}$ respectively; the subscript $\mathrm{m}$ is used to denote meridional components. The nearly axisymmetric dynamo operates provided that

$$
\begin{array}{ll}
\boldsymbol{u}^{* \prime}=\mathrm{O}\left(\varepsilon \overline{u_{\phi}^{*}}\right), & \overline{\boldsymbol{u}_{\mathrm{m}}^{*}}=\mathrm{O}\left(R m^{-1} \overline{u_{\phi}^{*}}\right), \\
\boldsymbol{b}^{* \prime}=\mathrm{O}\left(\varepsilon \overline{b_{\phi}^{*}}\right), & \overline{\boldsymbol{b}_{\mathrm{m}}^{*}}=\mathrm{O}\left(R m^{-1} \overline{b_{\phi}^{*}}\right)
\end{array}
$$

where the introduction of the small parameter

$$
\varepsilon \equiv R m^{-1 / 2}(\ell / \mathcal{L})^{1 / 2} \ll 1
$$

allows for the possibility that the fluctuations may occur on a shorter azimuthal length scale $\ell$. The normal situation, envisaged by Braginsky $(1964 \mathrm{a}, \mathrm{b})$, is $\ell=\mathrm{O}(\mathcal{L})$. On approximating the mean velocity $\overline{\boldsymbol{u}^{*}}$ and magnetic field $\overline{\boldsymbol{b}^{*}}$ by their dominant azimuthal parts $\overline{u_{\phi}^{*}} \widehat{\boldsymbol{\phi}}$ and $\overline{b_{\phi}^{*}} \widehat{\boldsymbol{\phi}}$, the fluctuating parts $\boldsymbol{u}^{* \prime}$ and $\boldsymbol{b}^{* \prime}$ given by (11c) and (19a) yield with the help of (47c) the leading order representations

$$
\begin{aligned}
\boldsymbol{u}^{* \prime} & \approx \partial_{t} \boldsymbol{\zeta}+\left(\overline{u_{\phi}^{*}} / s\right) \partial_{\phi} \boldsymbol{\zeta}-\left(s \boldsymbol{\zeta} \cdot \nabla\left(\overline{u_{\phi}^{*}} / s\right)\right) \widehat{\boldsymbol{\phi}} \\
\boldsymbol{b}^{* \prime} & \simeq\left(\overline{b_{\phi}^{*}} / s\right) \partial_{\phi} \boldsymbol{\zeta}-\left(s \boldsymbol{\zeta} \cdot \boldsymbol{\nabla}\left(\overline{b_{\phi}^{*}} / s\right)\right) \widehat{\boldsymbol{\phi}} .
\end{aligned}
$$

We continue to use the subscript $m$ to denote meridional parts: $\zeta_{\mathrm{m}}=\left(\zeta_{s}, 0, \zeta_{z}\right)$ and $\boldsymbol{u}_{\mathrm{m}}^{* \prime}=\left(u_{s}^{* \prime}, 0, u_{z}^{* \prime}\right), \quad \boldsymbol{b}_{\mathrm{m}}^{* \prime}=\left(b_{s}^{* \prime}, 0, b_{z}^{* \prime}\right)$. The latter are simply $\boldsymbol{u}_{\mathrm{m}}^{* \prime} \approx \partial_{t} \boldsymbol{\zeta}_{\mathrm{m}}+\left(\overline{u_{\phi}^{*}} / s\right) \partial_{\phi} \boldsymbol{\zeta}_{\mathrm{m}}$, $\boldsymbol{b}_{\mathrm{m}}^{* \prime} \simeq\left(\overline{b_{\phi}^{*}} / s\right) \partial_{\phi} \boldsymbol{\zeta}_{\mathrm{m}}$ with $\overline{\boldsymbol{u}^{*}} \simeq \overline{u_{\phi}^{*}} \widehat{\boldsymbol{\phi}}, \overline{\boldsymbol{b}^{*}} \simeq \overline{b_{\phi}^{*}} \widehat{\boldsymbol{\phi}}$ on the basis that $\overline{\boldsymbol{u}_{\mathrm{m}}^{*}}=\mathrm{O}\left(\zeta^{2} u_{\phi}^{*}\right), \overline{\boldsymbol{b}_{\mathrm{m}}^{*}}=\mathrm{O}\left(\zeta^{2} b_{\phi}^{*}\right)$. Provided that the time scale is not less than $\mathrm{O}(\ell / \mathcal{U}),(50 \mathrm{a}, \mathrm{b})$ are consistent with $(49 \mathrm{a}, \mathrm{c})$ when

$$
\zeta_{\mathrm{m}}=\mathrm{O}(\varepsilon \ell) \text {. }
$$

Braginsky (1964a) assumed steady flow for which $\partial_{t} \boldsymbol{\zeta}=\mathbf{0}$. Then the meridional component of $(50 \mathrm{a})$ can be integrated with respect to $\phi$ to obtain $\boldsymbol{\zeta}_{\mathrm{m}}$ (see Braginsky 1964a, where his key 
variables correspond to ours as follows: $v \mapsto \overline{u_{\phi}^{*}}, \boldsymbol{v}_{p}^{\prime} \mapsto \boldsymbol{u}_{\mathrm{m}}^{* \prime}, \boldsymbol{u}_{p}=\boldsymbol{v}_{p}{ }^{\prime} / v \mapsto s^{-1} \partial_{\phi}\left(\zeta_{s}, 0, \zeta_{z}\right)$ his eq. (3.11) and so $\widehat{\boldsymbol{u}}_{p} \mapsto s^{-1} \boldsymbol{\zeta}_{\mathrm{m}}$ on integration). In terms of $\boldsymbol{\zeta}_{\mathrm{m}}$, Braginsky introduced so-called effective-variables

$$
\begin{array}{ll}
\boldsymbol{u}_{\mathrm{m}}^{\dagger}=\nabla \times\left(\psi^{\dagger} \widehat{\boldsymbol{\phi}}\right), & \psi^{\dagger} \approx \psi^{*}+\varpi^{\ddagger} \overline{u_{\phi}^{*}}, \\
\overline{\boldsymbol{b}_{\mathrm{m}}^{\dagger}}=\nabla \times\left(a^{\dagger} \widehat{\boldsymbol{\phi}}\right), & a^{\dagger} \approx a^{*}+\varpi^{\ddagger} \overline{b_{\phi}^{*}},
\end{array}
$$

where, from our generalised form $(25 \mathrm{a}, \mathrm{b})$ and recalling that $\left[\boldsymbol{\zeta}, \boldsymbol{u}^{* \prime}\right]=-\boldsymbol{\nabla} \times\left(\boldsymbol{\zeta} \times \boldsymbol{u}^{* \prime}\right)$ for solenoidal $\boldsymbol{\zeta}$ and $\boldsymbol{u}^{* \prime}$ by (10a),

$$
\psi^{\dagger}-\psi^{*} \approx-\frac{1}{2}\left(\overline{\boldsymbol{\zeta}_{\mathrm{m}} \times \boldsymbol{u}_{\mathrm{m}}^{* \prime}}\right)_{\phi}, \quad \quad a^{\dagger}-a^{*} \approx-\frac{1}{2}\left(\overline{\boldsymbol{\zeta}_{\mathrm{m}} \times \boldsymbol{b}_{\mathrm{m}}^{* \prime}}\right)_{\phi} .
$$

So use of $(50 \mathrm{a}, \mathrm{b})$ determines

$$
\varpi^{\ddagger}=-\left(\overline{\boldsymbol{\zeta} \times \partial_{\phi} \boldsymbol{\zeta}}\right)_{\phi} / 2 s=-s^{-1} \overline{\zeta_{z} \partial_{\phi} \zeta_{s}}
$$

(see Braginsky 1964a, eq. (3.14): $w \mapsto \varpi^{\ddagger}$ ); the subscript ${ }_{\phi}$ denotes the $\phi$-component.

Here and below we use our notation $\boldsymbol{u}^{\dagger}$ and $\boldsymbol{b}^{\dagger}$ (see $(25 \mathrm{a}, \mathrm{b})$ ) for our generalisation of Braginsky's effective-variables. It is important to note however that, to the lowest order of accuracy achieved by Braginsky, the fluctuating effective-variable $\boldsymbol{b}^{\dagger \prime}$ may be ignored and so the distinction between $\boldsymbol{b}^{\dagger}$ and its average $\overline{\boldsymbol{b}}^{\dagger}$, introduced in section 1.3, is largely redundant as is the use of $\bumpeq$ and $\approx$. Moreover, whereas $\boldsymbol{u}_{\mathrm{m}}^{\dagger}$ and $\overline{\boldsymbol{b}_{\mathrm{m}}^{\dagger}}$ differ from $\overline{\boldsymbol{u}_{\mathrm{m}}^{*}}$ and $\overline{\boldsymbol{b}_{\mathrm{m}}^{*}}$ at lowest order, that is not the case for $u_{\phi}^{\dagger}=\overline{u_{\phi}^{*}}+\mathrm{O}\left((\zeta / \mathcal{L}) u_{\phi}^{* \prime}\right)$ and $\overline{b_{\phi}^{\dagger}}=\overline{b_{\phi}^{*}}+\mathrm{O}\left((\zeta / \mathcal{L}) b_{\phi}^{* \prime}\right)$ (see $\left.(25 \mathrm{a}, \mathrm{b})\right)$. As the discrepancies are small, $u_{\phi}^{\dagger} \simeq \overline{u_{\phi}^{*}}$ and $\overline{b_{\phi}^{\dagger}} \simeq \overline{b_{\phi}^{*}}$ may be regarded as leading order approximations to the mean azimuthal components of velocity and magnetic field. Indeed Braginsky worked with the latter quantities $\overline{u_{\phi}^{*}}$ and $\overline{b_{\phi}^{*}}$.

The azimuthal mean of (43a) governing the mean effective magnetic field $\boldsymbol{b}^{\dagger}$ may be decomposed into two equations governing the effective magnetic potential $a^{\dagger}$ and azimuthal magnetic field $\overline{b_{\phi}^{\dagger}}$. The approximate forms obtained by Braginsky are

$$
\begin{aligned}
& \partial_{t} a^{\dagger}+s^{-1} \boldsymbol{u}_{\mathrm{m}}^{\dagger} \cdot \boldsymbol{\nabla}\left(s a^{\dagger}\right) \doteqdot \mathfrak{K}\left(s^{-1} \aleph^{\ddagger} \overline{b_{\phi}^{\dagger}}+\Delta_{1} a^{\dagger}\right), \\
& \partial_{t} \overline{b_{\phi}^{\dagger}}+s \boldsymbol{u}_{\mathrm{m}}^{\dagger} \cdot \boldsymbol{\nabla}\left(s^{-1} \overline{b_{\phi}^{\dagger}}\right) \doteqdot s \overline{\boldsymbol{b}_{\mathrm{m}}^{\dagger}} \cdot \boldsymbol{\nabla}\left(s^{-1} u_{\phi}^{\dagger}\right)+\mathfrak{K} \Delta_{1} \overline{b_{\phi}^{\dagger}}
\end{aligned}
$$

(Braginsky 1964a, eq. (3.20) in which $D_{\mathrm{m}} \Gamma \mapsto \mathfrak{K} s^{-1} \aleph^{\ddagger}$ ), where

$$
\Delta_{1} \equiv \Delta-s^{-2}
$$

and the symbol $\doteqdot$ means correct to leading order as explained below (15a,b). The mean effective EMF $\mathcal{E}^{\dagger}$ (see (43b)) only appears in the system (52a,b) via its $\phi$-component

$$
-\mathfrak{K} J_{\phi}^{\dagger} \equiv \mathcal{E}_{\phi}^{\dagger} \doteqdot \mathfrak{K} s^{-1} \aleph^{\ddagger} \overline{b_{\phi}^{\dagger}} \quad\left(\overline{b_{\phi}^{\dagger}} \simeq \overline{b_{\phi}^{*}}\right)
$$

in (52a), the so called $\alpha$-effect. Braginsky (1964a) determines $-J_{\phi}^{\dagger} \doteqdot s^{-1} \aleph^{\ddagger} \overline{b_{\phi}^{*}}$ essentially from the formula (43c) with $\overline{\boldsymbol{b}^{*}}=\overline{b_{\phi}^{*}} \widehat{\boldsymbol{\phi}}$. It is not an easy calculation and he gives no hints how it should be accomplished. Nevertheless, since his result is linear in $\overline{b_{\phi}^{*}}$ and does not involve its derivatives, the value of $\aleph^{\ddagger}$ may be derived, in hindsight, directly from (43c) by the judicious choice $\overline{\boldsymbol{b}^{*}}=s \widehat{\boldsymbol{\phi}}$ for which $\Delta \overline{\boldsymbol{b}^{*}}=\mathbf{0}$ and $\boldsymbol{b}^{* \prime} \simeq \boldsymbol{\nabla} \times(s \boldsymbol{\zeta} \times \widehat{\boldsymbol{\phi}})=\partial_{\phi} \boldsymbol{\zeta}$ (see (47c)). Armed with these results, (43c) determines

$$
\begin{aligned}
\aleph^{\ddagger} & =\left(-\overline{\boldsymbol{\zeta} \times \Delta\left(\partial_{\phi} \boldsymbol{\zeta}\right)}+\frac{1}{2} \Delta\left(\overline{\boldsymbol{\zeta} \times\left(\partial_{\phi} \boldsymbol{\zeta}\right)}\right)\right)_{\phi} \\
& =\frac{1}{2}\left(-\overline{\boldsymbol{\zeta} \times \Delta\left(\partial_{\phi} \boldsymbol{\zeta}\right)}-\overline{\left(\partial_{\phi} \boldsymbol{\zeta}\right) \times(\Delta \boldsymbol{\zeta})}+\Delta\left(\overline{\boldsymbol{\zeta} \times\left(\partial_{\phi} \boldsymbol{\zeta}\right)}\right)\right)_{\phi}
\end{aligned}
$$


on use of $\partial_{\phi}(\overline{\boldsymbol{\zeta} \times \Delta \boldsymbol{\zeta}})=\mathbf{0}$,

$$
=\frac{1}{2}\left(-\left(\overline{\boldsymbol{\zeta}_{\mathrm{m}} \times\left(\Delta\left(\partial_{\phi} \boldsymbol{\zeta}\right)\right)_{\mathrm{m}}}\right)_{\phi}-\left(\overline{\left(\partial_{\phi} \boldsymbol{\zeta}_{\mathrm{m}}\right) \times(\Delta \boldsymbol{\zeta})_{\mathrm{m}}}\right)_{\phi}+\Delta_{1}\left(\overline{\boldsymbol{\zeta}_{\mathrm{m}} \times\left(\partial_{\phi} \boldsymbol{\zeta}_{\mathrm{m}}\right)}\right)_{\phi}\right) .
$$

Further reductions, that hinge on the identity $(\Delta \boldsymbol{\zeta})_{\mathrm{m}}=\left(\Delta_{1} \zeta_{s}-2 s^{-1} \partial_{\phi} \zeta_{\phi}, 0, \Delta \zeta_{z}\right)$, yield

$$
\aleph^{\ddagger}=2\left(\overline{\left(\nabla_{\mathrm{m}} \zeta_{z}\right) \cdot \nabla_{\mathrm{m}}\left(\partial_{\phi} \zeta_{s}\right)}+s^{-2} \overline{\left(\partial_{\phi} \zeta_{z}\right) \partial_{\phi}\left(\partial_{\phi} \zeta_{s}-\zeta_{\phi}\right)}\right)
$$

(Braginsky 1964a, eq. (3.21)), where, in view of the solenoidal condition $\boldsymbol{\nabla} \cdot \boldsymbol{\zeta}=0$ (see (11a))

$$
\partial_{\phi} \zeta_{\phi}=-s \nabla_{\mathrm{m}} \cdot \zeta_{\mathrm{m}}, \quad \nabla_{\mathrm{m}} \equiv\left(\partial_{s}, 0, \partial_{z}\right) .
$$

The $\omega$-effect in $(52 \mathrm{~b})$ provides the estimate $\left|\nabla a^{\dagger}\right| /\left|\overline{b_{\phi}^{\dagger}}\right|=\mathrm{O}\left(R m^{-1}\right)$, while the $\alpha$-effect in (52a) can support the mean meridional magnetic field, provided that $\left|s^{-1} \aleph^{\ddagger} b_{\phi}^{\dagger}\right| /\left|\Delta_{1} a^{\dagger}\right|=\mathrm{O}(1)$, which requires

$$
\aleph^{\ddagger}=\mathrm{O}\left(R m^{-1}\right) \text {. }
$$

This together with the the estimate $\left|\aleph^{\ddagger}\right|=\mathrm{O}\left(\mathcal{L} \zeta^{2} / \ell^{3}\right)$ implied by (54a) confirms the estimate (50c). Furthermore, since $\left|\varpi^{\ddagger}\right|=\mathrm{O}\left(\zeta^{2} / \ell\right)$, we deduce that

$$
\left|\nabla\left(\varpi^{\ddagger} \overline{b_{\phi}^{*}}\right)\right| /\left|\nabla a^{\dagger}\right|=\mathrm{O}\left((l / \mathcal{L})^{2}\right) .
$$

This means that the effective-variable corrections are significant when $\ell=\mathrm{O}(\mathcal{L})$, but are negligible in the case a two scale dynamo with $\ell \ll \mathcal{L}$.

Though we have explored the possibility of a short azimuthal length scale $\ell$, this is simply an embellishment of Braginsky's original model, for which there was a single length scale, namely the large length scale $\mathcal{L}$. His model is rather different from the standard two scale turbulent dynamo for which the $\alpha$-effect originates directly at leading order from approximating the mean EMF $\mathcal{E}^{*} \equiv \overline{\boldsymbol{u}^{* \prime} \times \boldsymbol{b}^{* \prime}}$ (see $(5 \mathrm{~b})$ ) by $\boldsymbol{\alpha}^{*} \cdot \overline{\boldsymbol{b}^{*}}$ (say). Under the single scale assumption $\ell=\mathcal{L}$, the leading order estimate for the magnitude of the azimuthal component of $\mathcal{E}^{*}$ is

$$
\mathcal{E}_{\phi}^{*} \equiv\left(\overline{\boldsymbol{u}_{\mathrm{m}}^{* \prime} \times \boldsymbol{b}_{\mathrm{m}}^{* \prime}}\right)_{\phi}=\mathrm{O}\left(R m^{-1}\left|\overline{\boldsymbol{u}^{*}}\right|\left|\overline{\boldsymbol{b}^{*}}\right|\right)
$$

on the basis that the sizes of $\boldsymbol{u}_{\mathrm{m}}^{* \prime}$ and $\boldsymbol{b}_{\mathrm{m}}^{* \prime}$ are given by (49a,c). Indeed the leading order value $\mathcal{E}_{\phi}^{*}=-\overline{E_{\phi}^{*}}-\left(\overline{\boldsymbol{u}_{\mathrm{m}}^{*}} \times \overline{\boldsymbol{b}_{\mathrm{m}}^{*}}\right)_{\phi}$ (see $\left.(22 \mathrm{~b})\right)$ is determined specifically by the $\phi$-components of $(26 \mathrm{a})$ and (27b), which give

$$
\begin{aligned}
\mathcal{E}_{\phi}^{*} & \doteqdot \frac{1}{2} \partial_{t}\left(\overline{\boldsymbol{\zeta}_{\mathrm{m}} \times \boldsymbol{b}_{\mathrm{m}}^{* \prime}}\right)_{\phi}+\left(\boldsymbol{u}_{\mathrm{m}}^{\dagger} \times \overline{\boldsymbol{b}_{\mathrm{m}}^{\dagger}}\right)_{\phi}-\left(\overline{\boldsymbol{u}_{\mathrm{m}}^{*}} \times \overline{\boldsymbol{b}_{\mathrm{m}}^{*}}\right)_{\phi} \\
& \doteqdot-\partial_{t}\left(a^{\dagger}-\overline{a^{*}}\right)+\left(\left(\boldsymbol{u}_{\mathrm{m}}^{\dagger}-\overline{\boldsymbol{u}_{\mathrm{m}}^{*}}\right) \times \overline{\boldsymbol{b}_{\mathrm{m}}^{*}}\right)_{\phi}+\left(\overline{\boldsymbol{u}_{\mathrm{m}}^{*}} \times\left(\overline{\boldsymbol{b}_{\mathrm{m}}^{\dagger}}-\overline{\boldsymbol{b}_{\mathrm{m}}^{*}}\right)\right)_{\phi}
\end{aligned}
$$

on use of (51f) (essentially as given by Braginsky 1964a, in the first line of his eq. (3.18) with the Ohmic diffusion terms proportional to $\mathfrak{K}$ omitted). The contribution (57b) is however absorbed within the mean magnetic induction equation by the choice of effective variables. Nevertheless with the diffusion terms retained, we are left with only the $\alpha$-term (52d):

$$
\mathcal{E}_{\phi}^{\dagger} \equiv-\mathfrak{K} J_{\phi}^{\dagger} \doteqdot \mathfrak{K} s^{-1} \aleph^{\ddagger} \overline{b_{\phi}^{\dagger}}=\mathrm{O}\left(R m^{-2}\left|\overline{\boldsymbol{u}^{*}}\right|\left|\overline{\boldsymbol{b}^{*}}\right|\right),
$$

since $R m=\mathcal{L}\left|\overline{\boldsymbol{u}^{*}}\right| / \mathfrak{K}$ and $\aleph^{\ddagger}=\mathrm{O}\left(R m^{-1}\right.$ ) (see (8a) and (55)), appearing in (52a). Usefully the second line of the expression (43c) for $J_{\phi}^{\dagger}$ vanishes, because the $\phi$-average of the $\phi$-component of gradients is zero. Of the remaining three terms in the first line of (43c), the last two terms, which determine

$$
-J_{\phi}^{\dagger}-\left(\nabla \times\left(\overline{\boldsymbol{b}_{\mathrm{m}}^{\dagger}}-\overline{\boldsymbol{b}_{\mathrm{m}}^{*}}\right)\right)_{\phi},
$$


correspond (in reverse order) to the terms in the second line of Braginsky (1964a) eq. (3.18), from which he derives the formula (54a) for $\aleph^{\ddagger}$. In powers of $R m^{-1 / 2},(58)$ is smaller than (57a) by two orders of magnitude! Therefore, the Braginsky dynamo is a low order dynamo, in the sense that both the zeroth and first orders contributions to $\mathcal{E}_{\phi}^{*}$ do not lead to a dynamo mechanism. It is only at second order that the the crucial dynamo ingredient emerges in the form of the $\alpha$-effect (58). That is why Braginsky's original calculations, which by necessity needed to be taken to second order, were a 'tour de force', as hinted at by the analysis in Appendix D. The small value of $\mathcal{E}_{\phi}^{\dagger}=-\mathfrak{K} \mathrm{J}_{\phi}^{\dagger}$ given by (58) is achieved because the azimuthal magnetic field $b_{\phi}^{*}$, which is weakly distorted by the fluctuating velocity $\boldsymbol{u}^{* \prime}$, remains largely aligned to total flow $\boldsymbol{u}^{*}$. Departures from this alignment only emerge after the magnetic field has diffused making its contribution $\mathrm{O}\left(\mathrm{Rm}^{-1}\right)$ smaller than originally anticipated. Herein lies the success of the HEL-approach. For whereas Braginsky's Eulerian derivation is cumbersome (even the evaluation of the result $-J_{\phi}^{\dagger} \doteqdot s^{-1} \aleph^{\ddagger} \overline{b_{\phi}^{\dagger}}$ from (43c) is awkward), HEL bypasses many of the difficulties by taking a coordinate system relative to which the distorted field (within the frozen field approximation) is actually axisymmetric! In this way the difficulty in evaluating $\mathcal{E}^{*} \equiv \overline{\boldsymbol{u}^{* \prime} \times \boldsymbol{b}^{* \prime}}$ correct to high order is bypassed and shifted to that of determining an appropriate mean of $\Delta \boldsymbol{b}^{*}$ in the new coordinate system, which leads to the effective EMF $\mathcal{E}_{\phi}^{\dagger} \doteqdot \mathfrak{K} s^{-1} \aleph^{\ddagger} \overline{b_{\phi}^{\dagger}}$ given by (58).

The complete 3-dimensional kinematic dynamo problem, as defined by (1a-h), was first solved numerically in the Braginsky limit by Kumar and Roberts (1975) and later by others (e.g, Sarson and Gubbins 1996, but see also Gubbins et al. 2000) and the results are in excellent agreement with those which they obtain from the 2-dimensional mean-field Braginsky equations $(52 \mathrm{a}, \mathrm{b})$.

\subsection{Outline of paper}

The essential difficulty with the Frieman and Rotenberg (1960) approach is the problem of identifying the correct choice of effective-variables at $\mathrm{O}\left(\zeta^{2}\right)$, as in the Braginsky $(1964 \mathrm{a}, \mathrm{b})$ dynamo. This is particularly true of the electric field $\boldsymbol{E}^{\dagger}$ as we have explained. The effectivevariables do however arise naturally from the HEL-approach (Soward 1972), which we explain in section 2. However, HEL itself also suffers the drawback of all Lagrangian approaches that the governing equations (see also section 4, including section 4.1 and Appendix E) at some position actually refer to conditions elsewhere (displaced by $\boldsymbol{\xi}$ ). As remarked in the Abstract and explained at the start of this introduction, Soward and Roberts (2010) overcame this difficulty through realising the Lagrangian displacement $\boldsymbol{\xi}$ by a 'fictitious steady flow' $\boldsymbol{\eta}$ in a unit of 'fictitious time'. Their Eulerian Transformed HEL (ETHEL) method builds on the pioneering ideas of Moffatt (1986) and corresponds to the 'Lie dragging' technique of general tensor calculus. Essentially the terms appearing in the HEL-equations are transformed into Eulerian (ETHEL) form (see sections 3 and 4.2,4.3) by means of Taylor expansions involving Lie derivatives.

The HEL-objective is to absorb the means of products, such as $\overline{\boldsymbol{u}^{* \prime} \times \boldsymbol{b}^{* \prime}}, \overline{\rho^{* \prime} \boldsymbol{u}^{* \prime}}$ and $\overline{\boldsymbol{u}^{* \prime} \cdot \boldsymbol{\nabla} \theta^{* \prime}}$, as much as possible into the product of means by the appropriate choice of HELvariables, just as the closely related effective-variables do in $(25 \mathrm{a}-\mathrm{d})$ correct to $\mathrm{O}\left(\zeta^{2}\right)$. In view of this close link, it is no real surprise to learn that the effective-variable development of this Introduction, summarising the results of Appendices $\mathrm{C}$ and D, actually builds in an essential way on properties of the Lie derivatives of the products $\boldsymbol{u}^{*} \times \boldsymbol{b}^{*}, \rho^{*} \boldsymbol{u}^{*}$ and $\boldsymbol{u}^{*} \cdot \boldsymbol{\nabla} \theta^{*}$ (see $($ B.9a-c)), while ETHEL generally simply invokes the Lie derivatives of individual variables in its Taylor series. Just like the effective-variable method, HEL shifts the difficulties associated with the mean transport terms into the small diffusion terms so far ignored. Andrews and McIntyre (1978a) refer to this as the Generalised Lagrangian Mean (GLM) approach. We 
must stress at the outset that only wave motion or wave turbulence is envisaged for which the restriction to small $\boldsymbol{\zeta}$ may be possible. Quite clearly the approach does not apply to situations such as fully developed turbulence for which $\zeta$ increases indefinitely

The key objective in section 5 is to cast those general results derived relative to rectangular Cartesian coordinates into cylindrical polar coordinates needed to describe the nearly axisymmetric Braginsky dynamo. That step is essential as the $\phi$-average of vectors is only meaningful when the average of their cylindrical polar coordinate components is taken. We develop the mean-field equations in section 5.1, provide a comprehensive study of the $\alpha$-effect in section 5.2 and Appendix F, and investigate briefly the role of Taylor's condition (Taylor 1963), as it pertains to the Braginsky expansion in section 5.3.

The non-local HEL-complication has been a continual irritation in the development of the subject. Indeed it motivated Holm (2002) to consider Eulerian means and led to his glm (as opposed to GLM) method. Likewise Vladimirov (2012) avoids HEL by constructing Eulerian means. Our point of view, is that Braginsky (1964a,b) achieved the glm-objective with his formulation of the mean-field dynamo equations (52a,b), involving effective-variables based on his $\widehat{\boldsymbol{u}}_{p}$ (our $s^{-1} \boldsymbol{\zeta}_{\mathrm{m}}$ ), albeit without introducing the Lagrangian concept. We advocate that the correct route to glm and effective-variables is via the ETHEL-method.

ETHEL formalises the effective-variable approach by building on an ETHEL-displacement $\boldsymbol{\eta}$, which differs slightly from both the HEL-displacement $\boldsymbol{\xi}$ and the effective-displacement $\boldsymbol{\zeta}$ at $\mathrm{O}\left(\eta^{2}\right)$, i.e. $\boldsymbol{\xi} \simeq \boldsymbol{\eta}$ and $\boldsymbol{\zeta} \simeq \boldsymbol{\eta}$. Perhaps, the most important feature of their construction is that we are able to conveniently demand that both $\zeta$ and $\boldsymbol{\eta}$ are fluctuating displacements with no mean parts: $\overline{\boldsymbol{\zeta}}=\overline{\boldsymbol{\eta}}=\mathbf{0}$. In section 3.2 , we refer to this assumption as

- $\quad$ the essential glm-ETHEL-postulate $\overline{\boldsymbol{\eta}}=\mathbf{0}$ (see (107d) and (195c)),

which we contrast in section 6 with

- the traditional GLM-HEL-postulate $\overline{\boldsymbol{\xi}}=\mathbf{0}$ (see (195a)).

Having demanded $\overline{\boldsymbol{\eta}}=\mathbf{0}$, the HEL-displacement $\boldsymbol{\xi}$ generally possesses a mean part $\overline{\boldsymbol{\xi}} \neq \mathbf{0}$. We stress, however, that though $\boldsymbol{\eta}$ and $\boldsymbol{\zeta}$ differ, our construction of $\dagger$ 'd effective-variables is based on the premise that they are actually HEL-variables (un- $\dagger^{\prime} d$ below), e.g. $\boldsymbol{u}^{\dagger} \equiv \boldsymbol{u}$. However, we treat our $\ddagger$ 'd variables, regarded as a functions of $\boldsymbol{\zeta}$ and $\boldsymbol{x}$, differently; their un- $\ddagger$ 'd ETHEL-versions are obtained on replacing $\boldsymbol{\zeta}$ by $\boldsymbol{\eta}$. Since $\boldsymbol{\zeta} \simeq \boldsymbol{\eta}$, the primary mean quantities satisfy $\aleph^{\ddagger} \approx \aleph, \varpi^{\ddagger} \approx \varpi$ with differences only emerging at $\mathrm{O}\left(\eta^{3}\right)$. These differences are negligible at $\mathrm{O}\left(\eta^{2}\right)$ of Braginsky's original calculations. In order to assess the implications of their discrepancy, we continue to the next order $\mathrm{O}\left(\eta^{3}\right)$ in analysis relegated to Appendix G. There we consider the ETHEL-expansions of $\overline{\boldsymbol{u}_{\mathrm{m}}^{*}}, \overline{\boldsymbol{b}_{\mathrm{m}}^{*}}$ in terms of $\boldsymbol{u}_{\mathrm{m}}\left(\equiv \boldsymbol{u}_{\mathrm{m}}^{\dagger}\right), \overline{\boldsymbol{b}_{\mathrm{m}}}\left(\equiv \overline{\boldsymbol{b}_{\mathrm{m}}^{\dagger}}\right)$. We compare their ETHEL-expansions as functions of $\boldsymbol{\eta}$, particularly $\varpi$, with the their effectivevariable expansions as functions of $\boldsymbol{\zeta}$, particularly $\varpi^{\ddagger}$, correct to $\mathrm{O}\left(\zeta^{3}\right)$ as derived previously by Tough (1967).

We summarise our findings in the concluding section 6 .

\section{The Hybrid Euler-Lagrange (HEL) method}

HEL itself differs from the Eulerian construction explained in section 1 and has its origins in the Lagrangian idea of the displacements $\boldsymbol{\xi}(\boldsymbol{x}, t)$ of fluid particles from a position P: $\boldsymbol{x}$ to a new position $\mathrm{P}^{L}: \boldsymbol{x}^{L}(\boldsymbol{x}, t)$ at time $t$, which moves with velocity $\boldsymbol{w}^{* L}(\boldsymbol{x}, t) \equiv \boldsymbol{w}^{*}\left(\boldsymbol{x}^{L}(\boldsymbol{x}, t)\right)$ :

$$
\boldsymbol{x}^{L}(\boldsymbol{x}, t)=\boldsymbol{x}+\boldsymbol{\xi}(\boldsymbol{x}, t), \quad \boldsymbol{w}^{* L}(\boldsymbol{x}, t)=\partial_{t} \boldsymbol{x}^{L}(\boldsymbol{x}, t)=\partial_{t} \boldsymbol{\xi}(\boldsymbol{x}, t) .
$$

In the case of shear flows this Lagrangian description is not helpful because neighbouring particle separate indefinitely. Nevertheless often in those situations, material lines themselves, 
such as frozen magnetic field lines, may remain close to some reference line. Then we choose $\mathrm{P}^{L}$ to be a point on the neighbouring material line close to $\mathrm{P}$ rather than the actual Lagrangian displaced material point. When $\boldsymbol{\xi}$ is small and under sensible assumptions, $\boldsymbol{\zeta}$ and $\boldsymbol{\xi}$ are almost the same: $\boldsymbol{\zeta} \simeq \boldsymbol{\xi}$. In that sense the linearised $\mathrm{O}(\xi)$ perfect fluid results (9a) and (19a-c) contain the rudiments of a HEL-construction following Frieman and Rotenberg (1960). However, the $\mathrm{O}\left(\xi^{2}\right)$ difference between $\boldsymbol{\zeta}$ and $\boldsymbol{\xi}$ needs to be addressed because it is at that order that means of the products of fluctuations appear in the definitions (25a-d) of the (perfect fluid) effectivevariables $\boldsymbol{u}^{\dagger}(\boldsymbol{x}, t), \boldsymbol{b}^{\dagger}(\boldsymbol{x}, t)$ and $\rho^{\dagger}(\boldsymbol{x}, t), \theta^{\dagger}(\boldsymbol{x}, t)$ (see also $\left.(26 \mathrm{a}, \mathrm{b})\right)$ governed by (27a-c), (28a,b) and (29). In the HEL-method one introduces new vector and scalar fields $\boldsymbol{u}(\boldsymbol{x}, t), \boldsymbol{b}(\boldsymbol{x}, t)$ and $\rho(\boldsymbol{x}, t), \theta(\boldsymbol{x}, t)$ that, in the perfect fluid case of small $\boldsymbol{\xi}$ envisaged above, are simply $\overline{\boldsymbol{u}^{*}}(\boldsymbol{x}, t)$, $\overline{\boldsymbol{b}^{*}}(\boldsymbol{x}, t)$ and $\overline{\rho^{*}}(\boldsymbol{x}, t), \overline{\theta^{*}}(\boldsymbol{x}, t)$ correct to $\mathrm{O}(\xi)$. Our aim is to link the HEL-variables to the effective-variables.

In the HEL-method, the coordinates $x_{i}$ provide the HEL-coordinates of a frame that at $\mathrm{P}^{L}: \boldsymbol{x}^{L}(\boldsymbol{x}, t)$ moves with the 'frame' velocity $\boldsymbol{w}^{* L}(\boldsymbol{x}, t)$. As in $(59 \mathrm{~b})$, we continue to use our notation that the superscript ${ }^{L}$ added to all scalar, vector and tensor quantities $\mathcal{T}^{*}$ denotes evaluation at $\mathrm{P}^{L}$ rather than $\mathrm{P}$ :

$$
\mathcal{T}^{* L}(\boldsymbol{x}, t) \equiv \mathcal{T}^{*}\left(\boldsymbol{x}^{L}(\boldsymbol{x}, t), t\right) .
$$

As explained in Part I (see also Soward and Roberts 2010) the frame velocity $\boldsymbol{w}^{*}$, magnetic field $\boldsymbol{b}^{*}$, density $\rho^{*}$ and temperature $\theta^{*}$ at $\mathrm{P}^{L}$ are expressed in the form

$$
\boldsymbol{w}^{* L}=\boldsymbol{w} \cdot \boldsymbol{\nabla} \boldsymbol{x}^{L}, \quad \boldsymbol{b}^{* L}=\mathcal{J}^{-1} \boldsymbol{b} \cdot \boldsymbol{\nabla} \boldsymbol{x}^{L}, \quad \rho^{* L}=\mathcal{J}^{-1} \rho, \quad \theta^{* L}=\theta \quad(61 \mathrm{a}-\mathrm{d})
$$

in terms of $\boldsymbol{w}(\boldsymbol{x}, t), \boldsymbol{b}(\boldsymbol{x}, t), \rho(\boldsymbol{x}, t)$ and $\theta(\boldsymbol{x}, t)$, where $\mathcal{J}$ is the Jacobian of the transformation $\boldsymbol{x} \mapsto \boldsymbol{x}^{L}:$

$$
\mathcal{J}=\left\|\nabla \boldsymbol{x}^{L}\right\|
$$

It is often useful to invert (61a-c) and write

$$
\boldsymbol{w}=\boldsymbol{w}^{* L} \cdot \nabla^{L} \boldsymbol{x}, \quad \boldsymbol{b}=\mathcal{J} \boldsymbol{b}^{* L} \cdot \nabla^{L} \boldsymbol{x}, \quad \rho=\mathcal{J} \rho^{* L} .
$$

Here, in component form, $\left(\nabla^{L} \boldsymbol{x}\right)_{j i} \equiv \nabla_{j}^{L} x_{i}$ and $x_{i}=x_{i}\left(\boldsymbol{x}^{L}, t\right)$, where

$$
\nabla_{j}^{L} \equiv \partial / \partial x_{j}^{L}, \quad \text { cf. } \quad \nabla_{i} \equiv \partial / \partial x_{i} .
$$

Moreover, $\boldsymbol{\nabla}^{L} \boldsymbol{x}$ is the inverse of $\boldsymbol{\nabla} \boldsymbol{x}^{L}$ :

$$
\left(\boldsymbol{\nabla} \boldsymbol{x}^{L}\right) \cdot\left(\boldsymbol{\nabla}^{L} \boldsymbol{x}\right)=\mathbf{I}=\left(\boldsymbol{\nabla}^{L} \boldsymbol{x}\right) \cdot\left(\boldsymbol{\nabla} \boldsymbol{x}^{L}\right) ; \quad(\mathbf{I})_{i j}=\delta_{i j},
$$

where $\left(\left(\boldsymbol{\nabla} \boldsymbol{x}^{L}\right) \cdot\left(\boldsymbol{\nabla}^{L} \boldsymbol{x}\right)\right)_{i j} \equiv\left(\boldsymbol{\nabla} \boldsymbol{x}^{L}\right)_{i k}\left(\boldsymbol{\nabla}^{L} \boldsymbol{x}\right)_{k j}$. In order to dispel any notational ambiguity, we define the scalar product involving second rank Cartesian tensors by demanding that the subscript that naturally occurs before and after the 'dot' is repeated and contracted upon.

We diverge from our Part I and Soward and Roberts (2010) notation for the velocity itself in a crucial way by transforming the relative velocity $\boldsymbol{u}^{* L}-\boldsymbol{w}^{* L}$ (true velocity $\boldsymbol{u}^{*}$ less frame velocity $\boldsymbol{w}^{*}$ ) and write

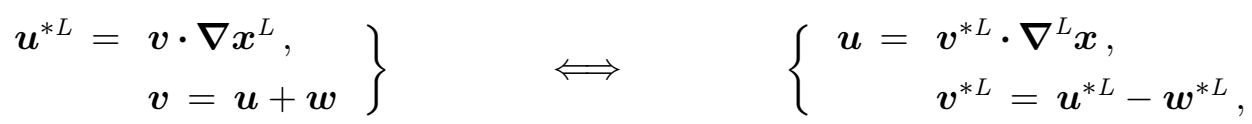

which are equivalent because $\boldsymbol{w}^{* L}=\boldsymbol{w} \cdot \boldsymbol{\nabla} \boldsymbol{x}^{L}$ and $\boldsymbol{w}=\boldsymbol{w}^{* L} \cdot \nabla^{L} \boldsymbol{x}$ (see (61a) and (62a)). Though the meaning of $\boldsymbol{u}, \boldsymbol{v}, \boldsymbol{w}$ and $\boldsymbol{w}^{*}$ is the same as in Part I, here we have implemented the interchange $\boldsymbol{u}^{*} \longleftrightarrow \boldsymbol{v}^{*}$. We emphasise that, though our $\boldsymbol{v}$ and $\boldsymbol{v}^{*}$ are useful in setting up the initial transformations, they are peripheral to the final HEL-formulation. An important consequence of the definition $\boldsymbol{u}^{* L}=\boldsymbol{w}^{* L}+\boldsymbol{u} \cdot \boldsymbol{\nabla} \boldsymbol{x}^{L}$ (see (65a)), where $\boldsymbol{w}^{* L}=\partial_{t} \boldsymbol{x}^{L}=\partial_{t} \boldsymbol{\xi}$ (see $(59 \mathrm{~b}, \mathrm{a}))$, is that

$$
\boldsymbol{u}^{* L}=\mathrm{D}_{t} \boldsymbol{x}^{L}=\boldsymbol{u}+\mathrm{D}_{t} \xi \quad \text { with } \quad \mathrm{D}_{t} \equiv \partial_{t}+\boldsymbol{u} \cdot \boldsymbol{\nabla} .
$$


Also central, in what follows in the later sections, are the alternative representations

$$
\boldsymbol{V}^{* L}=\left(\boldsymbol{\nabla}^{L} \boldsymbol{x}\right) \cdot \boldsymbol{V}, \quad \boldsymbol{H}^{* L}=\left(\boldsymbol{\nabla}^{L} \boldsymbol{x}\right) \cdot \boldsymbol{H}, \quad\left(\boldsymbol{\nabla} \theta^{*}\right)^{L}=\left(\boldsymbol{\nabla}^{L} \boldsymbol{x}\right) \cdot(\boldsymbol{\nabla} \theta),
$$

where $\boldsymbol{V}^{*}=\boldsymbol{u}^{*}$ and $\mu \boldsymbol{H}^{*}=\boldsymbol{b}^{*}$. So with $(62 \mathrm{a}, \mathrm{b})$ we have, using $(64 \mathrm{a}, \mathrm{b})$, that

$$
\left|\boldsymbol{u}^{* L}\right|^{2}=\boldsymbol{u}^{* L} \cdot \boldsymbol{V}^{* L}=\boldsymbol{v} \cdot \boldsymbol{V}, \quad \mu^{-1}\left|\boldsymbol{b}^{* L}\right|^{2}=\boldsymbol{b}^{* L} \cdot \boldsymbol{H}^{* L}=\boldsymbol{b} \cdot \boldsymbol{H},
$$

and

$$
\boldsymbol{v}=\boldsymbol{g} \cdot \boldsymbol{V}, \quad \boldsymbol{b}=\mu \mathcal{J} \boldsymbol{g} \cdot \boldsymbol{H}
$$

where

$$
(\boldsymbol{g})_{i j} \equiv g_{i j}=\left(\nabla_{k}^{L} x_{i}\right)\left(\nabla_{k}^{L} x_{j}\right) .
$$

As we expand upon in the following section 2.1, $\boldsymbol{V}$ and $\boldsymbol{w}$ correspond to covariant and contravariant vectors of the general tensor calculus, which we distinguish by our use of upper and lower case vectors. Since $\mathrm{d} \boldsymbol{x}=\mathrm{d} \boldsymbol{x}^{L} \cdot \nabla^{L} \boldsymbol{x}$, they have the properties

$$
\mathrm{d} \boldsymbol{x} \cdot \boldsymbol{V}=\mathrm{d} \boldsymbol{x}^{L} \cdot \boldsymbol{V}^{* L}, \quad \boldsymbol{w} \cdot \boldsymbol{\nabla}=\boldsymbol{w}^{* L} \cdot \boldsymbol{\nabla}^{L} .
$$

The curl and vector product operations involve the alternating tensor $\left(\boldsymbol{\epsilon}^{*}\right)_{i j k} \equiv \epsilon_{i j k}^{*}$ in the Cartesian reference frame at $\mathrm{P}^{L}$ with the important properties

$$
\epsilon_{i j k}^{*}=\mathcal{J}^{-1} \epsilon_{l m n}\left(\nabla_{l} x_{i}^{L}\right)\left(\nabla_{m} x_{j}^{L}\right)\left(\nabla_{n} x_{k}^{L}\right) \quad \text { or } \quad \epsilon_{i j k}^{*}=\mathcal{J}\left(\nabla_{i}^{L} x_{l}\right)\left(\nabla_{j}^{L} x_{m}\right)\left(\nabla_{k}^{L} x_{n}\right) \epsilon_{l m n},
$$

in which the transformed tensor $\left(\epsilon^{*} \mapsto\right) \epsilon$ at $\mathrm{P}$ has components

$$
(\boldsymbol{\epsilon})_{i j k} \equiv \epsilon_{i j k}=\epsilon_{i j k}^{*}
$$

it is contravariant in the former (70a) (see Part I, eq. (A.3b)) and covariant in the latter (70b). A useful consequence of (70a) is

$$
\mathcal{J} \epsilon_{i j k}^{*} \nabla_{k}^{L} x_{n}=\epsilon_{l m n}\left(\nabla_{l} x_{i}^{L}\right)\left(\nabla_{m} x_{j}^{L}\right)
$$

which on differentiation yields

$$
\epsilon_{i j k}^{*} \nabla_{n}\left(\mathcal{J} \nabla_{k}^{L} x_{n}\right)=0 \quad \Longleftrightarrow \quad \nabla_{n}\left(\mathcal{J} \nabla_{k}^{L} x_{n}\right)=0
$$

(see Part I, eq. (A.4e)).

As an example of the use of (70a), we consider the electric current $\boldsymbol{j}^{*}=\boldsymbol{\nabla} \times \boldsymbol{H}^{*}$, which at $\mathrm{P}^{L}$ has components

$$
\begin{aligned}
j_{i}^{* L}=\epsilon_{i j k}^{*} \nabla_{j}^{L} H_{k}^{* L} & =\mathcal{J}^{-1} \epsilon_{l m n}\left(\nabla_{l} x_{i}^{L}\right)\left(\nabla_{n} x_{k}^{L}\right) \nabla_{m} H_{k}^{* L} \\
& =\mathcal{J}^{-1}\left(\epsilon_{l m n} \nabla_{m}\left(\left(\nabla_{n} x_{k}^{L}\right) H_{k}^{* L}\right)\right)\left(\nabla_{l} x_{i}^{L}\right)=\mathcal{J}^{-1}\left(\epsilon_{l m n} \nabla_{m} H_{n}\right)\left(\nabla_{l} x_{i}^{L}\right),
\end{aligned}
$$

aided by $(67 \mathrm{~b})$. In vector form it says that

$$
\boldsymbol{j}^{* L}=\left(\boldsymbol{\nabla} \times \boldsymbol{H}^{*}\right)^{L}=\mathcal{J}^{-1} \boldsymbol{j} \cdot \boldsymbol{\nabla} \boldsymbol{x}^{L}, \quad \text { where } \quad \boldsymbol{j}=\boldsymbol{\nabla} \times \boldsymbol{H}
$$

is contravariant. Likewise, on use of (70b), the EMF and Lorentz force are

$$
\left(\boldsymbol{u}^{*} \times \boldsymbol{b}^{*}\right)^{L}=\left(\boldsymbol{\nabla}^{L} \boldsymbol{x}\right) \cdot(\boldsymbol{v} \times \boldsymbol{b}), \quad\left(\boldsymbol{j}^{*} \times \boldsymbol{b}^{*}\right)^{L}=\mathcal{J}^{-1}\left(\boldsymbol{\nabla}^{L} \boldsymbol{x}\right) \cdot(\boldsymbol{j} \times \boldsymbol{b})
$$

(but see Part I, eqs. (19), (26b)). Combinations of the above results are possible. So, for example, on making the variable changes $\boldsymbol{H}^{* L} \mapsto\left(\boldsymbol{u}^{*} \times \boldsymbol{b}^{*}\right)^{L}, \boldsymbol{H} \mapsto \boldsymbol{v} \times \boldsymbol{b}$ in (67b), compatible with (73a), the formulae $(72 b, c)$ determine

$$
\left(\boldsymbol{\nabla} \times\left(\boldsymbol{u}^{*} \times \boldsymbol{b}^{*}\right)\right)^{L}=\mathcal{J}^{-1}(\boldsymbol{\nabla} \times(\boldsymbol{v} \times \boldsymbol{b})) \cdot \boldsymbol{\nabla} \boldsymbol{x}^{L} .
$$

Another useful result is obtained from the replacements of $\boldsymbol{j}^{*} \mapsto \boldsymbol{\nabla} \times \boldsymbol{V}^{*}, \boldsymbol{j} \mapsto \boldsymbol{\nabla} \times \boldsymbol{V}$ and $\boldsymbol{b}^{*} \mapsto \boldsymbol{u}^{*}, \boldsymbol{b} \mapsto \mathcal{J} \boldsymbol{v}$ in (73b) with $\boldsymbol{H}^{*} \mapsto \boldsymbol{V}^{*}, \boldsymbol{H} \mapsto \boldsymbol{V}, \boldsymbol{b}^{*} \mapsto \boldsymbol{u}^{*}, \boldsymbol{b} \mapsto \mathcal{J} \boldsymbol{v}$ in (72b,c):

$$
\left(\left(\boldsymbol{\nabla} \times \boldsymbol{V}^{*}\right) \times \boldsymbol{u}^{*}\right)^{L}=\left(\boldsymbol{\nabla}^{L} \boldsymbol{x}\right) \cdot((\boldsymbol{\nabla} \times \boldsymbol{V}) \times \boldsymbol{v}) .
$$


At $\mathrm{P}^{L}, \boldsymbol{\nabla}^{*} \cdot \boldsymbol{b}^{*}$ takes the value

$$
\nabla_{k}^{L} b_{k}^{* L}=\left(\nabla_{k}^{L} x_{n}\right)\left(\nabla_{n} b_{k}^{* L}\right)=\mathcal{J}^{-1} \nabla_{n}\left(\mathcal{J} b_{k}^{* L} \nabla_{k}^{L} x_{n}\right)=\mathcal{J}^{-1} \nabla_{n} b_{n}
$$

since $\nabla_{n}\left(\mathcal{J} \nabla_{k}^{L} x_{n}\right)=0$ (see (71c)). Following the respective replacements $\boldsymbol{b} \mapsto \mathcal{J} \boldsymbol{v} \mapsto \rho \boldsymbol{v} \mapsto$ $\mathcal{J} \boldsymbol{g} \cdot \boldsymbol{\nabla} \theta$, we obtain the divergence properties

$$
\begin{aligned}
\left(\boldsymbol{\nabla} \cdot \boldsymbol{u}^{*}\right)^{L} & =\mathcal{J}^{-1} \boldsymbol{\nabla} \cdot(\mathcal{J} \boldsymbol{v}), & \left(\boldsymbol{\nabla} \cdot\left(\rho^{*} \boldsymbol{u}^{*}\right)\right)^{L} & =\mathcal{J}^{-1} \boldsymbol{\nabla} \cdot(\rho \boldsymbol{v}), \\
\left(\boldsymbol{\nabla} \cdot \boldsymbol{b}^{*}\right)^{L} & =\mathcal{J}^{-1} \boldsymbol{\nabla} \cdot \boldsymbol{b}, & \left(\Delta \theta^{*}\right)^{L} & =\mathcal{J}^{-1} \boldsymbol{\nabla} \cdot(\mathcal{J} \boldsymbol{g} \cdot \boldsymbol{\nabla} \theta),
\end{aligned}
$$

in which $(75 \mathrm{a}, \mathrm{d})$ are equivalent. Some related transformations of the gradient of a vector are given by (A.1a,b) of Appendix A.

\subsection{Time derivatives}

It is important to realise the time derivative $\partial_{t} \theta^{* L}$ of a scalar quantity $\theta^{*}$ at the moving point $\mathrm{P}^{L}: \boldsymbol{x}^{L}(\boldsymbol{x}, t)$ is not the same as its time derivative $\left(\partial_{t} \theta^{*}\right)^{L}$ at $\mathrm{P}^{L}$. Instead, since $\theta^{* L}(\boldsymbol{x}, t)=\theta^{*}\left(\boldsymbol{x}^{L}(\boldsymbol{x}, t), t\right)$ (see (60)), we have $\partial_{t} \theta^{* L}=\left(\partial_{t} \theta^{*}\right)^{L}+\left(\partial_{t} \boldsymbol{x}^{L}\right) \cdot \nabla^{L} \theta^{* L}$, which with $\partial_{t} \boldsymbol{x}^{L}=\boldsymbol{w} \cdot \boldsymbol{\nabla} \boldsymbol{x}^{L}$ determines

$$
\left(\partial_{t} \theta^{*}\right)^{L}=\left(\partial_{t}-\boldsymbol{w} \cdot \nabla\right) \theta^{* L}=\left(\partial_{t}-\boldsymbol{w} \cdot \nabla\right) \theta
$$

since $\theta^{* L}=\theta($ see $(61 \mathrm{~d}))$. Combined with $\boldsymbol{u}^{* L} \cdot \nabla^{L}(=\boldsymbol{v} \cdot \boldsymbol{\nabla})=(\boldsymbol{u}+\boldsymbol{w}) \cdot \boldsymbol{\nabla}$ implied by $(65 \mathrm{a})$, the result (76a) determines

$$
\left(\partial_{t} \theta^{*}+\boldsymbol{u}^{*} \cdot \boldsymbol{\nabla} \theta^{*}\right)^{L}=\left(\partial_{t}+\boldsymbol{u} \cdot \boldsymbol{\nabla}\right) \theta=\mathrm{D}_{t} \theta
$$

(see $(66 \mathrm{~b}))$.

We extend the above idea for scalars to tensor quantities $\mathcal{T}^{*}$ (see (60)) in Appendix A. There we find that the transformation of $\partial_{t} \mathcal{T}^{*}$ (see (A.4a)) involves the Lie derivative, whose form (A.5) depends on the general tensor character (contravariant, covariant and weight w) of $\mathcal{T}$. In application of (A.4a) to the governing equations, we need

$$
\begin{aligned}
\mathcal{J}\left(\partial_{t} \boldsymbol{b}^{*}\right)^{L} \cdot \boldsymbol{\nabla}^{L} \boldsymbol{x} & =\partial_{t} \boldsymbol{b}-\mathrm{L}_{\boldsymbol{w}} \boldsymbol{b}, & \mathrm{L}_{\boldsymbol{w}} \boldsymbol{b} & =\llbracket \boldsymbol{w}, \boldsymbol{b} \rrbracket, \\
\left(\boldsymbol{\nabla} \boldsymbol{x}^{L}\right) \cdot\left(\partial_{t} \boldsymbol{V}^{*}\right)^{L} & =\partial_{t} \boldsymbol{V}-\mathrm{L}_{\boldsymbol{w}} \boldsymbol{V}, & \mathrm{L}_{\boldsymbol{w}} \boldsymbol{V} & =\{\boldsymbol{w}, \boldsymbol{V}\}, \\
\mathcal{J}\left(\partial_{t} \rho^{*}\right)^{L} & =\partial_{t} \rho-\mathrm{L}_{\boldsymbol{w}} \rho, & \mathrm{L}_{\boldsymbol{w}} \rho & =\boldsymbol{\nabla} \cdot(\rho \boldsymbol{w}), \\
\left(\partial_{t} \theta^{*}\right)^{L} & =\partial_{t} \theta-\mathrm{L}_{\boldsymbol{w}} \theta, & \mathrm{L}_{\boldsymbol{w}} \theta & =\boldsymbol{w} \cdot \boldsymbol{\nabla} \rho,
\end{aligned}
$$

where the various Lie derivatives $\mathrm{L}_{\boldsymbol{w}}$ in the right-hand column are derived from (A.5) on the basis that contravariant $\boldsymbol{b}$ and $\rho$ have weight $\mathrm{w}=-1$ and covariant $\boldsymbol{V}$ and $\theta$ are unweighted. Furthermore, since $\boldsymbol{b}$ is solenoidal (see (75b) or Part I, eq. (16a)), (77b) has the alternative and more useful representation

$$
\llbracket \boldsymbol{w}, \boldsymbol{b} \rrbracket=-\boldsymbol{\nabla} \times(\boldsymbol{w} \times \boldsymbol{b}), \quad \text { since } \quad \boldsymbol{\nabla} \cdot \boldsymbol{b}=0 .
$$

Likewise application of (A.7) with the replacements $\boldsymbol{w}^{*} \mapsto \boldsymbol{u}^{*}$ and $\boldsymbol{w} \mapsto \boldsymbol{v}$, since $\boldsymbol{u}^{* L}=$ $\boldsymbol{v} \cdot \boldsymbol{\nabla} \boldsymbol{x}^{L}$ (see (65b)), determines the following Lie derivative transformations:

$$
\begin{aligned}
& \mathcal{J}\left(\mathrm{L}_{\boldsymbol{u}^{*}} \boldsymbol{b}^{*}\right)^{L} \cdot \boldsymbol{\nabla}^{L} \boldsymbol{x}=\mathrm{L}_{\boldsymbol{v}} \boldsymbol{b} \quad \Longrightarrow \quad \mathcal{J}\left(\partial_{t} \boldsymbol{b}^{*}+\mathrm{L}_{\boldsymbol{u}^{*}} \boldsymbol{b}^{*}\right)^{L} \cdot \boldsymbol{\nabla}^{L} \boldsymbol{x}=\partial_{t} \boldsymbol{b}+\mathrm{L}_{\boldsymbol{u}} \boldsymbol{b}, \\
& \left(\boldsymbol{\nabla} \boldsymbol{x}^{L}\right) \cdot\left(\mathrm{L}_{\boldsymbol{u}^{*}} \boldsymbol{V}^{*}\right)^{L}=\mathrm{L}_{\boldsymbol{v}} \boldsymbol{V} \Longrightarrow\left(\boldsymbol{\nabla} \boldsymbol{x}^{L}\right) \cdot\left(\partial_{t} \boldsymbol{V}^{*}+\mathrm{L}_{\boldsymbol{u}^{*}} \boldsymbol{V}^{*}\right)^{L}=\partial_{t} \boldsymbol{V}+\mathrm{L}_{\boldsymbol{u}} \boldsymbol{V}, \\
& \mathcal{J}\left(\mathrm{L}_{\boldsymbol{u}^{*} \rho^{*}}\right)^{L}=\mathrm{L}_{\boldsymbol{v}} \rho \quad \Longrightarrow \quad \mathcal{J}\left(\partial_{t} \rho^{*}+\mathrm{L}_{\boldsymbol{u}^{*} \rho^{*}}\right)^{L}=\partial_{t} \rho+\mathrm{L}_{\boldsymbol{u}} \rho, \\
& \left(\mathrm{L}_{\boldsymbol{u}^{*}} \theta^{*}\right)^{L}=\mathrm{L}_{\boldsymbol{v}} \theta \quad \Longrightarrow \quad\left(\partial_{t} \theta^{*}+\mathrm{L}_{\boldsymbol{u}^{*}} \theta^{*}\right)^{L}=\partial_{t} \theta+\mathrm{L}_{\boldsymbol{u}} \theta
\end{aligned}
$$


where the right-hand column follows from $(77 \mathrm{a}-\mathrm{h})$ on the basis that $\mathrm{L}_{\boldsymbol{v}}-\mathrm{L}_{\boldsymbol{w}}=\mathrm{L}_{\boldsymbol{u}}$, since $\boldsymbol{v}-\boldsymbol{w}=\boldsymbol{u}$ (see (65b) again).

Application of $(79 \mathrm{~b})$ to the magnetic induction equations $(1 \mathrm{a}, \mathrm{b}, \mathrm{d})$ illustrates its importance

$$
\partial_{t} \boldsymbol{b}^{*}+\mathrm{L}_{\boldsymbol{u}^{*}} \boldsymbol{b}^{*}=\boldsymbol{\nabla} \times \boldsymbol{J}^{*} \quad \Longrightarrow \quad \partial_{t} \boldsymbol{b}+\mathrm{L}_{\boldsymbol{u}} \boldsymbol{b}=\boldsymbol{\nabla} \times \boldsymbol{J},
$$

in which we have used $\mathcal{J}\left(\boldsymbol{\nabla} \times \boldsymbol{J}^{*}\right)^{L} \cdot \nabla^{L} \boldsymbol{x}=\boldsymbol{\nabla} \times \boldsymbol{J}$, where

$$
\boldsymbol{J}^{* L}=\left(\boldsymbol{\nabla}^{L} \boldsymbol{x}\right) \cdot \boldsymbol{J} \quad \Longleftrightarrow \quad \boldsymbol{J}=\left(\boldsymbol{\nabla} \boldsymbol{x}^{L}\right) \cdot \boldsymbol{J}^{* L},
$$

a result that follows from (72b,c) and (67b) after the variable changes $\boldsymbol{H} \mapsto \boldsymbol{J}$ and $\boldsymbol{H}^{*} \mapsto \boldsymbol{J}^{*}$. Similar benefits follow from expressing the equations of mass continuity (see $(2 \mathrm{a}, \mathrm{b}))$ and heat conduction (3a) in the form

$$
\begin{array}{llrl}
\partial_{t} \rho^{*}+\mathrm{L}_{\boldsymbol{u}^{*}} \rho^{*}=0 & \Longrightarrow & \partial_{t} \rho+\mathrm{L}_{\boldsymbol{u}} \rho=0 \\
\partial_{t} \theta^{*}+\mathrm{L}_{\boldsymbol{u}^{*}} \theta^{*}=\kappa \Delta \theta^{*}+q^{*} & \Longrightarrow & \partial_{t} \theta+\mathrm{L}_{\boldsymbol{u}} \theta=\kappa \mathcal{J}^{-1} \boldsymbol{\nabla} \cdot(\mathcal{J} \boldsymbol{g} \cdot \boldsymbol{\nabla} \theta)+q
\end{array}
$$

(see $(75 \mathrm{e}))$, where

$$
q^{* L}=q .
$$

\section{$2.2 \quad$ Perfect electrical conduction}

For the special case of a perfectly electrically conducting $\left(\sigma^{-1}=0 \Longrightarrow \boldsymbol{J}=\mathbf{0}\right)$ and thermally insulating $(\kappa=0)$ fluid without heat sources $\left(q^{*}=0\right)$ discussed in section 1.2 , the system (80b), (82b,d) becomes

$$
\begin{aligned}
& \partial_{t} \boldsymbol{b}=-\boldsymbol{\nabla} \times \boldsymbol{E}, \\
& \boldsymbol{E}=-\boldsymbol{u} \times \boldsymbol{b}, \\
& \boldsymbol{\nabla} \cdot \boldsymbol{b}=0, \\
& \partial_{t} \rho=-\nabla \cdot \boldsymbol{m}, \\
& \boldsymbol{m}=\rho \boldsymbol{u}, \\
& \mathrm{D}_{t} \theta=0
\end{aligned}
$$

(cf. Part I, eqs. (13d), (16a), (20) and (22b)), where

$$
\begin{array}{lll}
\boldsymbol{E}^{* L}=\left(\boldsymbol{\nabla}^{L} \boldsymbol{x}\right) \cdot(\boldsymbol{E}-\boldsymbol{w} \times \boldsymbol{b}) & \Longleftrightarrow & \boldsymbol{E}=\left(\boldsymbol{\nabla} \boldsymbol{x}^{L}\right) \cdot\left(\boldsymbol{E}^{*}+\boldsymbol{w}^{*} \times \boldsymbol{b}^{*}\right)^{L}, \\
\boldsymbol{m}^{* L}=\mathcal{J}^{-1}(\boldsymbol{m}+\rho \boldsymbol{w}) \cdot \boldsymbol{\nabla} \boldsymbol{x}^{L} & \Longleftrightarrow & \boldsymbol{m}=\mathcal{J}\left(\boldsymbol{m}^{*}-\rho^{*} \boldsymbol{w}^{*}\right)^{L} \cdot \boldsymbol{\nabla}^{L} \boldsymbol{x}
\end{array}
$$

(cf. $(65 \mathrm{a}, \mathrm{b}))$ :

$$
\boldsymbol{u}^{* L}=(\boldsymbol{u}+\boldsymbol{w}) \cdot \boldsymbol{\nabla} \boldsymbol{x}^{L} \quad \Longleftrightarrow \quad \boldsymbol{u}=\left(\boldsymbol{u}^{*}-\boldsymbol{w}^{*}\right)^{L} \cdot \nabla^{L} \boldsymbol{x} .
$$

The equivalence of $(84 \mathrm{a}, \mathrm{b})$ and $(84 \mathrm{c}, \mathrm{d})$ depends on the properties $\left(\boldsymbol{w}^{*} \times \boldsymbol{b}^{*}\right)^{L}=\left(\nabla^{L} \boldsymbol{x}\right) \cdot(\boldsymbol{w} \times \boldsymbol{b})$ (see $(73 \mathrm{a}))$ and $\left(\rho^{*} \boldsymbol{w}^{*}\right)^{L}=\mathcal{J}^{-1} \rho \boldsymbol{w} \cdot \boldsymbol{\nabla} \boldsymbol{x}^{L}($ see $(61 \mathrm{a}, \mathrm{c}))$.

When the point $\mathrm{P}^{L}$ is stationary implying $\boldsymbol{w}^{* L}=\mathbf{0}$, the vectors $\boldsymbol{E}=\left(\boldsymbol{\nabla} \boldsymbol{x}^{L}\right) \cdot \boldsymbol{E}^{* L}$ (the definition of $\boldsymbol{E}$ chosen in Part I, their eq. (18)), $\boldsymbol{m}=\mathcal{J}\left(\boldsymbol{\nabla} \boldsymbol{x}^{L}\right) \cdot \boldsymbol{m}^{* L}$ and $\boldsymbol{u}=\left(\boldsymbol{\nabla} \boldsymbol{x}^{L}\right) \cdot \boldsymbol{u}^{* L}$ transform according to the usual tensor transformation rules. However, when $\mathrm{P}^{L}$ moves, our choices $(84 \mathrm{~b}, \mathrm{~d}, \mathrm{e})$ are more natural, as they builds on the electric field $\left(\boldsymbol{E}^{*}+\boldsymbol{w}^{*} \times \boldsymbol{b}^{*}\right)^{L}$, mass flux $\left(\boldsymbol{m}^{*}-\rho^{*} \boldsymbol{w}^{*}\right)^{L}$ and velocity $\left(\boldsymbol{u}^{*}-\boldsymbol{w}^{*}\right)^{L}$ at $\mathrm{P}^{L}$ in the frame moving with velocity $\boldsymbol{w}^{* L}$. It follows from (61b) and (84c) that the mass $\boldsymbol{m}^{*} \cdot \mathrm{d} \boldsymbol{S}^{*}$ and magnetic $\boldsymbol{b}^{*} \cdot \mathrm{d} \boldsymbol{S}^{*}$ fluxes across any vector element of surface $\mathrm{d} \boldsymbol{S}^{* L}$, composed of points $\mathrm{P}^{L}$ fixed in space not moving with $\boldsymbol{w}^{* L}$, satisfy

$$
\left.\begin{array}{rl}
\boldsymbol{m}^{* L} \cdot \mathrm{d} \boldsymbol{S}^{* L} & =(\boldsymbol{m}+\rho \boldsymbol{w}) \cdot \mathrm{d} \boldsymbol{S}, \\
\boldsymbol{b}^{* L} \cdot \mathrm{d} \boldsymbol{S}^{* L} & =\boldsymbol{b} \cdot \mathrm{d} \boldsymbol{S},
\end{array}\right\} \quad \mathrm{d} \boldsymbol{S}=\mathcal{J}^{-1}\left(\boldsymbol{\nabla} \boldsymbol{x}^{L}\right) \cdot \mathrm{d} \boldsymbol{S}^{* L},
$$

where $\mathrm{d} \boldsymbol{S}=\mathrm{d} \boldsymbol{x}^{(1)} \times \mathrm{d} \boldsymbol{x}^{(2)}$ and $\mathrm{d} \boldsymbol{S}^{* L}=\mathrm{d} \boldsymbol{x}^{* L(1)} \times \mathrm{d} \boldsymbol{x}^{* L(2)}$ (cf. Part I, eqs. (12b), (16b) for $\boldsymbol{b}$ ). When instead the surface $\mathrm{d} \boldsymbol{S}^{* L}$ moves, i.e. the points P that compose $\mathrm{d} \boldsymbol{S}$ are fixed, then the mass flux relative to the moving surface $\mathrm{d} \boldsymbol{S}^{* L}$ is $\left(\boldsymbol{m}^{* L}-\rho \boldsymbol{w}^{* L}\right) \cdot \mathrm{d} \boldsymbol{S}^{* L}=\boldsymbol{m} \cdot \mathrm{d} \boldsymbol{S}$. 
The way by which we take advantage of the HEL-formulation is to demand that $\boldsymbol{u}$ has no fluctuating part (i.e. $\boldsymbol{u}=\overline{\boldsymbol{u}}$ ) for all time $t$. This possibility exists through absorbing fluctuations of the motion by the HEL-displacement $\boldsymbol{\xi}$. An immediate consequence is that, if there are no fluctuating parts to $\rho, \boldsymbol{b}$ and $\theta$ at the initial instant, there are no fluctuating parts for all time (i.e. $\rho=\bar{\rho}, \boldsymbol{b}=\overline{\boldsymbol{b}}$ and $\theta=\bar{\theta}$ ). These are the ideas behind the HEL-formulation by Soward (1972) of Braginsky's nearly axisymmetric dynamo.

\subsection{Finite electrical conductivity}

The implementation of HEL in the Braginsky nearly axisymmetric dynamo context is to assume that the magnetic diffusion though small is finite, so that the electric field $\boldsymbol{E}^{*}$ in Faraday's law (1a) is determined by Ohm's law (1b). Accordingly, though the HEL-equation (83a) still holds, we replace the HEL electric field (83b) by

$$
\boldsymbol{E}=-\boldsymbol{u} \times \boldsymbol{b}+\boldsymbol{J}
$$

for which summing the equalities (73a) and (84a) determines

$$
\left(\boldsymbol{E}^{*}+\boldsymbol{u}^{*} \times \boldsymbol{b}^{*}\right)^{L}=\left(\boldsymbol{\nabla}^{L} \boldsymbol{x}\right) \cdot(\boldsymbol{E}+\boldsymbol{u} \times \boldsymbol{b}) .
$$

In (86a) we have $\boldsymbol{J}=\mathfrak{K}\left(\boldsymbol{\nabla} \boldsymbol{x}^{L}\right) \cdot\left(\boldsymbol{\nabla} \times \boldsymbol{b}^{*}\right)^{L}$ (see (81b) and (1g)). So with the help of the identity $\left(\nabla_{i} x_{q}^{L}\right) \epsilon_{q m p}=\epsilon_{i k l}\left(\nabla_{m}^{L} x_{k}\right)\left(\nabla_{p}^{L} x_{l}\right)$, which follows from (70b) with $\mathcal{J}=1$ (or see Part I, eq. (A.4b)), the component form of $\mathfrak{K}^{-1} \boldsymbol{J}$ is

$$
\begin{aligned}
\mathfrak{K}^{-1} J_{i} & =\left(\nabla_{i} x_{q}^{L}\right) \epsilon_{q m p}\left(\nabla_{m} \boldsymbol{b}_{p}^{*}\right)^{L}=\mathcal{J} \epsilon_{i k l}\left(\nabla_{m}^{L} x_{k}\right)\left(\nabla_{p}^{L} x_{l}\right)\left(\nabla_{m} \boldsymbol{b}_{p}^{*}\right)^{L} \\
& =\mathcal{J} \epsilon_{i k l}\left(\nabla_{m}^{L} x_{k}\right)\left(\nabla_{m}^{L}\left(\boldsymbol{b}^{* L} \cdot \nabla^{L} x_{l}\right)-\boldsymbol{b}^{* L} \cdot \nabla^{L}\left(\nabla_{m}^{L} x_{l}\right)\right),
\end{aligned}
$$

on use of $\nabla_{m}^{L}\left(\nabla_{p}^{L} x_{l}\right)=\nabla_{p}^{L}\left(\nabla_{m}^{L} x_{l}\right)$,

$$
\begin{aligned}
& =\epsilon_{i k l}\left(\nabla_{m}^{L} x_{k}\right)\left(\mathcal{J} \nabla_{m}^{L}\left(\mathcal{J}^{-1} b_{l}\right)-\boldsymbol{b} \cdot \boldsymbol{\nabla}\left(\nabla_{m}^{L} x_{l}\right)\right) \\
& =\epsilon_{i k l}\left(\mathcal{J} g_{k n} \nabla_{n}\left(\mathcal{J}^{-1} b_{l}\right)-\left(\nabla_{m}^{L} x_{k}\right) \boldsymbol{b} \cdot \boldsymbol{\nabla}\left(\nabla_{m}^{L} x_{l}\right)\right),
\end{aligned}
$$

where $g_{i j}=(\boldsymbol{g})_{i j}$ is given by (68). More compactly (87) may be written as

$$
\mathfrak{K}^{-1} \boldsymbol{J}=(\boldsymbol{g} \cdot \boldsymbol{\nabla}) \times \boldsymbol{b}-\boldsymbol{\alpha} \cdot \boldsymbol{b},
$$

(see Part I, eq. (23b) with the sign of $\boldsymbol{\alpha}$ reversed to conform with conventional notation), where the components $\alpha_{i j}=(\boldsymbol{\alpha})_{i j}$ are given by

$$
\begin{aligned}
\alpha_{i j} & =\epsilon_{i k l}\left(\nabla_{m}^{L} x_{k}\right) \nabla_{j}\left(\nabla_{m}^{L} x_{l}\right)-\epsilon_{i k j}\left(\nabla_{m}^{L} x_{k}\right)\left(\nabla_{m}^{L} x_{l}\right)\left(\mathcal{J}_{l} \mathcal{J}^{-1}\right) \\
& =\left(\nabla_{m}^{L} x_{k}\right)\left(\epsilon_{i k l} \nabla_{j}-\epsilon_{i k j} \nabla_{l}\right)\left(\nabla_{m}^{L} x_{l}\right),
\end{aligned}
$$

on use of $\nabla_{l}\left(\mathcal{J} \nabla_{m}^{L} x_{l}\right)=0$ (see $\left.(71 \mathrm{c})\right)$,

$$
=\left(\nabla_{m}^{L} x_{k}\right)\left(\epsilon_{k l j} \nabla_{i}-\epsilon_{i l j} \nabla_{k}\right)\left(\nabla_{m}^{L} x_{l}\right),
$$

on use of (B.1c). Forming the sum $\frac{1}{2}((89 b)+(89 c))$ determines

$$
\alpha_{i j}=\frac{1}{2}\left(\breve{\alpha}_{i j}+\breve{\alpha}_{j i}\right)+\frac{1}{2} \epsilon_{i j k} \nabla_{l} g_{l k}, \quad \text { where } \quad \breve{\alpha}_{i j}=\left(\nabla_{m}^{L} x_{k}\right) \epsilon_{k l i} \nabla_{j}\left(\nabla_{m}^{L} x_{l}\right)
$$

and the anti-symmetric contribution to $\alpha_{i j}$ satisfies

$$
\epsilon_{i j k} \alpha_{j k}=\nabla_{j} g_{i j} \text {. }
$$

Now unlike $\boldsymbol{b}$ which has no fluctuating part in the high conductivity limit, the covariant vector $\boldsymbol{J}$ does (see (88)) and this in turn forces a small fluctuating contribution to $\boldsymbol{b}$ via (83a) and (86a). The crucial approximation that renders the HEL-approach useful in the Braginsky context is that we may simply make the leading order approximations $\boldsymbol{b} \doteq \overline{\boldsymbol{b}}$ and $\boldsymbol{J} \doteqdot \overline{\boldsymbol{J}}$, as the fluctuating parts are relatively small: $\boldsymbol{b}^{\prime}=\mathrm{O}\left(R m^{-1} \eta|\overline{\boldsymbol{b}}|\right)$ and $\boldsymbol{J}^{\prime}=\mathrm{O}(\eta|\overline{\boldsymbol{J}}|)$. 


\section{The Euler transformed HEL-method (ETHEL)}

Already (61a-d) have exposed an unfortunate feature of the HEL-method, namely that, though $\rho(\boldsymbol{x}, t), \boldsymbol{u}(\boldsymbol{x}, t), \boldsymbol{b}(\boldsymbol{x}, t)$ and $\theta(\boldsymbol{x}, t)$ are all functions identified by the point $\mathrm{P}: \boldsymbol{x}$, they describe conditions elsewhere at $\mathrm{P}^{L}: \boldsymbol{x}^{L}(\boldsymbol{x}, t)$. Indeed motivated by this shortcoming, Holm (2002) searched for an Eulerian development which incorporated features of the HEL-theory. In the case of small displacements $\xi$, we may address the issue by considering the Taylor series expansions typified by that for the temperature field $\theta^{*}$ at $\mathrm{P}^{L}$ :

$$
\theta(\boldsymbol{x}, t)=\theta^{* L}(\boldsymbol{x}, t) \equiv \theta^{*}(\boldsymbol{x}+\boldsymbol{\xi}, t)=\left(\theta^{*}+\boldsymbol{\xi} \cdot \boldsymbol{\nabla} \theta^{*}+\frac{1}{2}(\boldsymbol{\xi} \boldsymbol{\xi}):(\boldsymbol{\nabla} \nabla) \theta^{*}+\cdots\right)(\boldsymbol{x}, t),
$$

which provides a representation of the HEL-variable $\theta$ with coordinates $x_{i}$ (so describing $\theta^{*}$ at $\mathrm{P}^{L}$; see (61d)) in terms of $\theta^{*}$ at $\mathrm{P}$. The expansion (91a) inverts awkwardly to give the temperature field $\theta^{*}$ at $\mathrm{P}$ :

$$
\theta^{*}(\boldsymbol{x}, t)=\left(\theta-\left(\boldsymbol{\xi}-\frac{1}{2} \boldsymbol{\xi} \cdot \boldsymbol{\nabla} \boldsymbol{\xi}\right) \cdot \boldsymbol{\nabla} \theta+\frac{1}{2} \boldsymbol{\xi} \cdot \boldsymbol{\nabla}(\boldsymbol{\xi} \cdot \boldsymbol{\nabla} \theta)-\cdots\right)(\boldsymbol{x}, t),
$$

in terms of the HEL-variable $\theta$ also evaluated at P. In this way the HEL-equations governing the HEL-variables $\rho, \boldsymbol{u}, \boldsymbol{b}$ and $\theta$ may be recast in terms of their Eulerian counterparts $\rho^{*}$, $\boldsymbol{v}^{*}, \boldsymbol{b}^{*}$ and $\theta^{*}$. Soward and Roberts (2010) refer to this approach as Euler transformed HEL (ETHEL).

As the step from (91a) to (91b) suggests, the implementation of ETHEL using the HELdisplacement $\boldsymbol{\xi}$ is rather unwieldy in the case of $\theta$, and even more so for $\rho, \boldsymbol{v}$ and $\boldsymbol{b}$. To simplify matters, we employ a technique introduced by Moffatt (1986), whereby the mapping $\boldsymbol{x} \mapsto \boldsymbol{x}^{L}$ is achieved by dragging $\mathrm{P}$ to $\mathrm{P}^{L}$ by a 'fictitious steady flow' $\boldsymbol{\eta}^{*}(\boldsymbol{x}, t)$ in a unit of 'fictitious time' $\tau$ (say; below we will be more explicit writing $\tau$-steady to mean independent of $\tau$ at fixed $\boldsymbol{x})$ via intermediate points $\mathrm{P}^{\ell}: \boldsymbol{x}^{\ell}(\boldsymbol{x}, t, \tau)$, at which the 'fictitious velocity' is $\boldsymbol{\eta}^{* \ell}=\boldsymbol{\eta}^{*}\left(\boldsymbol{x}^{\ell}, t\right)$ dependent on $\tau$ via $\boldsymbol{x}^{\ell}$, for $0<\tau<1$ with $\boldsymbol{x}^{\ell}(\boldsymbol{x}, t, 0)=\boldsymbol{x}$ and $\boldsymbol{x}^{\ell}(\boldsymbol{x}, t, 1)=\boldsymbol{x}^{L}$. Our approach differs in one subtle respect from Moffatt's original implementation of the method. Whereas, he envisaged that the physical state of the system changed as a result of the dragging, i.e. the movement of frozen magnetic field, we regard the physical state as fixed but the coordinates as changing. It is this alternative interpretation that enabled Soward and Roberts (2010) to generalise the approach and to identify the technique with the 'Lie dragging' of general tensor calculus (for an elementary account, see d'Inverno 1992). The essential idea (see Soward and Roberts 2010, section 3.1, also Schutz 1980, equation (2.6)) is that $\boldsymbol{x}^{\ell}$ and the corresponding $\mathcal{J}^{\ell}$ have Taylor series expansions

$$
\begin{aligned}
& \boldsymbol{x}^{\ell}(\tau)=\boldsymbol{x}+\tau\left(\partial_{\tau} \boldsymbol{x}^{\ell}\right)_{\tau=0}+\frac{1}{2} \tau^{2}\left(\partial_{\tau}^{2} \boldsymbol{x}^{\ell}\right)_{\tau=0}+\frac{1}{3 !} \tau^{3}\left(\partial_{\tau}^{3} \boldsymbol{x}^{\ell}\right)_{\tau=0}+\cdots, \\
& \mathcal{J}^{\ell}(\tau)=1+\tau\left(\partial_{\tau} \mathcal{J}^{\ell}\right)_{\tau=0}+\frac{1}{2} \tau^{2}\left(\partial_{\tau}^{2} \mathcal{J}^{\ell}\right)_{\tau=0}+\frac{1}{3 !} \tau^{3}\left(\partial_{\tau}^{3} \mathcal{J}^{\ell}\right)_{\tau=0}+\cdots
\end{aligned}
$$

about $\tau=0$, recall that $\boldsymbol{x}^{\ell}(0)=\boldsymbol{x}$ and $\mathcal{J}^{\ell}(0)=1$. Since, at fixed $t, \boldsymbol{x}^{\ell}(\boldsymbol{x}, \tau)$ behaves like $\boldsymbol{x}^{L}(\boldsymbol{x}, t)$, we may utilise our earlier kinematic results (59b), (61a) and (A.2d) under the change of variables $\boldsymbol{w}^{*} \mapsto \boldsymbol{\eta}^{*}, t \mapsto \tau, \boldsymbol{x}^{L} \mapsto \boldsymbol{x}^{\ell}$ (more generally ${ }^{L} \mapsto^{\ell}$ ) and $\mathcal{J} \mapsto \mathcal{J}^{\ell}$ to show that $\boldsymbol{x}^{\ell}$ and $\mathcal{J}^{\ell}$ satisfy

$$
\partial_{\tau} \boldsymbol{x}^{\ell}=\boldsymbol{\eta}^{* \ell}=\boldsymbol{\eta} \cdot \boldsymbol{\nabla} \boldsymbol{x}^{\ell}, \quad \partial_{\tau} \mathcal{J}^{\ell}=\boldsymbol{\nabla} \cdot\left(\mathcal{J}^{\ell} \boldsymbol{\eta}\right)
$$

respectively. Here we have introduced the 'fictitious HEL $\tau$-steady flow' $(\boldsymbol{w} \mapsto) \boldsymbol{\eta}(\boldsymbol{x}, t)$, which by (93a) has the interesting $\tau=0$ and $\tau=1$ properties

$$
\boldsymbol{\eta}^{*}=\boldsymbol{\eta}, \quad \boldsymbol{\eta}^{* L}=\boldsymbol{\eta} \cdot \boldsymbol{\nabla} \boldsymbol{x}^{L} .
$$

The latter (94b) identifies $\boldsymbol{\eta}$ as the HEL-value of the Eulerian 'fictitious $\tau$-steady flow' $\boldsymbol{\eta}^{*}$, which realises the displacement $\boldsymbol{\xi}$. The former (94a) makes the powerful statement that $\boldsymbol{\eta}^{*}$ and $\boldsymbol{\eta}$ are the same. Henceforth we will only use $\boldsymbol{\eta}$ and call it the ETHEL-displacement; though 
it is helpful to bear in mind its simultaneous Eulerian and HEL-interpretation. The $\tau=1$ values of $\boldsymbol{x}^{\ell}$ and $\mathcal{J}^{\ell}$ given by $(92 \mathrm{a}, \mathrm{b})$ determine

$$
\begin{aligned}
& \boldsymbol{\xi}(\boldsymbol{x}, t)=\boldsymbol{x}^{L}-\boldsymbol{x}=\boldsymbol{\eta}+\frac{1}{2} \boldsymbol{\eta} \cdot \boldsymbol{\nabla} \boldsymbol{\eta}+\frac{1}{3 !}(\boldsymbol{\eta} \cdot \boldsymbol{\nabla})^{2} \boldsymbol{\eta}+\cdots \\
& \mathcal{J}(\boldsymbol{x}, t)-1=\boldsymbol{\nabla} \cdot \boldsymbol{\eta}+\frac{1}{2} \boldsymbol{\nabla} \cdot((\boldsymbol{\nabla} \cdot \boldsymbol{\eta}) \boldsymbol{\eta})+\frac{1}{3 !} \boldsymbol{\nabla} \cdot((\boldsymbol{\nabla} \cdot((\boldsymbol{\nabla} \cdot \boldsymbol{\eta}) \boldsymbol{\eta})) \boldsymbol{\eta})+\cdots
\end{aligned}
$$

Likewise, the $\tau=1$ values of the corresponding Taylor series for $\boldsymbol{\nabla} \boldsymbol{x}^{\ell}$ and $\boldsymbol{\nabla}^{\ell} \boldsymbol{x}$, which satisfy $\partial_{\tau}\left(\boldsymbol{\nabla} \boldsymbol{x}^{\ell}\right)=\left\{\boldsymbol{\eta}, \boldsymbol{\nabla} \boldsymbol{x}^{\ell}\right\}$ and $\partial_{\tau}\left(\boldsymbol{\nabla}^{\ell} \boldsymbol{x}\right)=\left[\boldsymbol{\eta}, \boldsymbol{\nabla}^{\ell} \boldsymbol{x}\right]$ as in (A.2a,b) (also $\left.\boldsymbol{\nabla} \boldsymbol{x}^{\ell}(0)=\boldsymbol{\nabla}^{\ell} \boldsymbol{x}(0)=\mathbf{I}\right)$, leads to

$$
\begin{aligned}
\boldsymbol{\nabla} \boldsymbol{x}^{L} & =\mathbf{I}+\boldsymbol{\nabla} \boldsymbol{\eta}+\frac{1}{2}\{\boldsymbol{\eta}, \boldsymbol{\nabla} \boldsymbol{\eta}\}+\cdots, & \{\boldsymbol{\eta}, \mathbf{I}\} & =\boldsymbol{\nabla} \boldsymbol{\eta}, \\
\boldsymbol{\nabla}^{L} \boldsymbol{x} & =\mathbf{I}-\boldsymbol{\nabla} \boldsymbol{\eta}-\frac{1}{2}[\boldsymbol{\eta}, \boldsymbol{\nabla} \boldsymbol{\eta}]-\cdots, & {[\boldsymbol{\eta}, \mathbf{I}] } & =-\boldsymbol{\nabla} \boldsymbol{\eta} .
\end{aligned}
$$

\subsection{Lie derivative expansions}

The same method can be applied to general tensors $\mathcal{T}$ taking the value $\mathcal{T}^{\ell}$ at $\mathrm{P}^{\ell}: \boldsymbol{x}^{\ell}(\boldsymbol{x}, t, \tau)$ and possessing the Taylor series expansions

$$
\mathcal{T}^{\ell}(\tau)=\mathcal{T}^{*}+\tau\left(\partial_{\tau} \mathcal{T}^{\ell}\right)_{\tau=0}+\frac{1}{2} \tau^{2}\left(\partial_{\tau}^{2} \mathcal{T}^{\ell}\right)_{\tau=0}+\frac{1}{3 !} \tau^{3}\left(\partial_{\tau}^{3} \mathcal{T}^{\ell}\right)_{\tau=0}+\cdots
$$

about $\tau=0$, recall that $\mathcal{T}^{\ell}(0)=\mathcal{T}^{*}$. To that end, we build on the general tensor results of Appendix A, where upper and lower indices are used to denote contravariant, $\mathcal{T}^{i}$, and covariant, $\mathcal{T}_{i}$, vectors respectively. There, in addition to the change of variables $t \mapsto \tau, \boldsymbol{w} \mapsto \boldsymbol{\eta}$ made in (93a,b), we make the further variable change $\mathcal{T} \mapsto \mathcal{T}^{\ell}$ in (A.4a). Then noting that the physical state $\mathcal{T}^{*}$ is independent of $\tau$, we have $\partial_{\tau} \mathcal{T}^{*}=\mathbf{0}$ implying $\left(\partial_{\tau} \mathcal{T}^{*}\right)^{\ell}=\mathbf{0}$ also. Accordingly the left-hand side of (A.4b) vanishes implying

$$
\partial_{\tau} \mathcal{T}^{\ell}=\mathrm{L}_{\boldsymbol{\eta}} \mathcal{T}^{\ell}
$$

Substitution into (97a) and evaluation at $\tau=1$, where $\mathcal{T}^{\ell}(1)=\mathcal{T}$, determines

$$
\mathcal{T}(\boldsymbol{x}, t)=\left(\exp \mathrm{L}_{\boldsymbol{\eta}}\right) \mathcal{T}^{*}(\boldsymbol{x}, t) \equiv \mathcal{T}^{*}+\mathrm{L}_{\boldsymbol{\eta}} \mathcal{T}^{*}+\frac{1}{2}\left(\mathrm{~L}_{\boldsymbol{\eta}}\right)^{2} \mathcal{T}^{*}+\frac{1}{3 !}\left(\mathrm{L}_{\boldsymbol{\eta}}\right)^{2} \mathcal{T}^{*}+\cdots
$$

It should be noted that the Lie derivative in our Taylor expansions evaluated at $\tau=0$, for which the ${ }^{\ell}$-frame coincides with the Cartesian frame, may lead to a notational ambiguity as there the contravariant and covariant forms of vectors are indistinguishable in the sense that $\mathcal{T}^{* i}=\mathcal{T}_{i}^{*}$. For that reason we prefer to state results for the inverse Taylor series expansion

$$
\mathcal{T}^{*}(\boldsymbol{x}, t)=\left(\exp \left(-\mathrm{L}_{\boldsymbol{\eta}}\right)\right) \mathcal{T}(\boldsymbol{x}, t) \equiv \boldsymbol{T}-\mathrm{L}_{\boldsymbol{\eta}} \mathcal{T}+\frac{1}{2}\left(\mathrm{~L}_{\boldsymbol{\eta}}\right)^{2} \mathcal{T}-\frac{1}{3 !}\left(\mathrm{L}_{\boldsymbol{\eta}}\right)^{2} \mathcal{T}-\cdots
$$

in which $\mathrm{L}_{\boldsymbol{\eta}} \mathcal{T}$ is uniquely defined. That said, their difference yields the useful expansion

$$
2\left(\mathcal{T}-\mathcal{T}^{*}\right)=\mathrm{L}_{\boldsymbol{\eta}} \widetilde{\mathcal{T}}-\frac{1}{2} \mathrm{~L}_{\boldsymbol{\eta}}^{2}\left(\mathcal{T}-\mathcal{T}^{*}\right)+\cdots, \quad \widetilde{\mathcal{T}} \equiv \mathcal{T}^{*}+\mathcal{T}
$$

The Lie derivatives $\mathrm{L}_{\boldsymbol{\eta}}$ of $\boldsymbol{b}, \boldsymbol{V}, \rho, \theta$ (also $q=q^{* L}$ ) are given by (77b,d,f,h). Analogously, after $\boldsymbol{w} \mapsto \boldsymbol{\eta}$, the corresponding Lie derivatives of some frequently appearing tensors of different 
weights w are

$$
\begin{array}{llll}
\mathrm{L}_{\boldsymbol{\eta}} \mathcal{T} \equiv(\boldsymbol{\eta} \cdot \boldsymbol{\nabla}) \mathcal{T}, & \text { scalars, } & \mathrm{w}=0, & \mathcal{T}=\theta, \boldsymbol{u} \cdot \boldsymbol{\nabla} \theta, q, \\
\mathrm{~L}_{\boldsymbol{\eta}} \mathcal{T} \equiv \boldsymbol{\nabla} \cdot(\mathcal{T} \boldsymbol{\eta}), & \text { scalars, } & \mathrm{w}=-1, & \mathcal{T}=\rho, \\
\mathrm{L}_{\boldsymbol{\eta}} \mathcal{T} \equiv[\boldsymbol{\eta}, \mathcal{T}], & \text { contravariant vectors, }, & \mathrm{w}=0, & \mathcal{T}=\boldsymbol{u}, \boldsymbol{w}, \boldsymbol{\eta}, \\
\mathrm{L}_{\boldsymbol{\eta}} \mathcal{T} \equiv \llbracket \boldsymbol{\eta}, \mathcal{T} \rrbracket, & \text { contravariant vectors, } & \mathrm{w}=-1, & \mathcal{T}=\boldsymbol{b}, \boldsymbol{j}, \boldsymbol{m}, \rho \boldsymbol{u}, \rho \boldsymbol{w}, \\
\mathrm{L}_{\boldsymbol{\eta}} \mathcal{T} \equiv\{\boldsymbol{\eta}, \mathcal{T}\}, & \text { covariant vectors }{ }^{1}, & \mathrm{w}=0, & \mathcal{T}=\boldsymbol{V}, \boldsymbol{\nabla} \theta, \boldsymbol{H}, \boldsymbol{J}, \\
& & \boldsymbol{E}, \boldsymbol{u} \times \boldsymbol{b}, \boldsymbol{w} \times \boldsymbol{b} .
\end{array}
$$

Some examples (B.9a-c) of the Lie derivatives of products are established from first principles in Appendix B and are used in Appendices C and D to establish the Eulerian results outlined in section 1. For solenoidal contravariant vectors $\boldsymbol{b}$ (see (78b)), $\boldsymbol{j}$ (see (72c)), (99d) possesses the alternative representation

$$
\llbracket \boldsymbol{\eta}, \mathcal{T} \rrbracket=-\boldsymbol{\nabla} \times(\boldsymbol{\eta} \times \mathcal{T}), \quad \text { when } \quad \boldsymbol{\nabla} \cdot \mathcal{T}=0
$$

(see, e.g., (78a,b)). Interestingly, the ETHEL-displacement $\mathcal{T}=\boldsymbol{\eta}$ in (99c) satisfies

$$
\mathrm{L}_{\boldsymbol{\eta}} \boldsymbol{\eta} \equiv[\boldsymbol{\eta}, \boldsymbol{\eta}]=\mathbf{0} .
$$

Thus the same substitution $\mathcal{T}=\boldsymbol{\eta}$ into (98b) recovers the identity $\boldsymbol{\eta}^{*}=\boldsymbol{\eta}$ (see (94a)).

\subsubsection{The frame velocity $\boldsymbol{w}$}

With $\mathrm{L}_{\boldsymbol{\eta}} \bullet=[\boldsymbol{\eta}, \bullet]($ as in $(99 \mathrm{c}))$, we also need

$$
\begin{gathered}
\boldsymbol{w}(\boldsymbol{x}, t)=\partial_{t} \boldsymbol{\eta}+\frac{1}{2} \mathrm{~L}_{\boldsymbol{\eta}}\left(\partial_{t} \boldsymbol{\eta}\right)+\frac{1}{3 !}\left(\mathrm{L}_{\boldsymbol{\eta}}\right)^{2}\left(\partial_{t} \boldsymbol{\eta}\right)+\cdots \\
\boldsymbol{w}^{*}(\boldsymbol{x}, t)=\partial_{t} \boldsymbol{\eta}-\frac{1}{2} \mathrm{~L}_{\boldsymbol{\eta}}\left(\partial_{t} \boldsymbol{\eta}\right)+\frac{1}{3 !}\left(\mathrm{L}_{\boldsymbol{\eta}}\right)^{2}\left(\partial_{t} \boldsymbol{\eta}\right)-\cdots
\end{gathered}
$$

(see Soward and Roberts 2010, eqs. (3.30a,b)), whose sum and difference yield odd and even series in $\boldsymbol{\eta}$ with leading order terms

$$
\widetilde{\boldsymbol{w}} \equiv \boldsymbol{w}^{*}+\boldsymbol{w} \approx 2 \partial_{t} \boldsymbol{\eta}, \quad \boldsymbol{w}-\boldsymbol{w}^{*} \approx\left[\boldsymbol{\eta}, \partial_{t} \boldsymbol{\eta}\right]
$$

possessing error terms $\mathrm{O}\left(\eta^{3}\right)$ and $\mathrm{O}\left(\eta^{4}\right)$ (whence $\approx$, see $(13 \mathrm{c})$ ) respectively. For tensors $\mathcal{T}$, $\mathcal{T}^{*}$ and $\widetilde{\mathcal{T}}=\mathcal{T}^{*}+\mathcal{T}$ that satisfy $\mathcal{T}-\mathcal{T}^{*}=\mathrm{O}(\eta)$, the properties (103a,b) imply that

$$
\begin{aligned}
& \boldsymbol{w} \mathcal{T}+\boldsymbol{w}^{*} \mathcal{T}^{*}=\frac{1}{2} \widetilde{\boldsymbol{w}} \widetilde{\mathcal{T}}+\frac{1}{2}\left(\boldsymbol{w}-\boldsymbol{w}^{*}\right)\left(\mathcal{T}-\mathcal{T}^{*}\right) \approx\left(\partial_{t} \boldsymbol{\eta}\right) \widetilde{\mathcal{T}} \\
& \boldsymbol{w} \mathcal{T}-\boldsymbol{w}^{*} \mathcal{T}^{*}=\frac{1}{2} \widetilde{\boldsymbol{w}}\left(\mathcal{T}-\mathcal{T}^{*}\right)+\frac{1}{2}\left(\boldsymbol{w}-\boldsymbol{w}^{*}\right) \widetilde{\mathcal{T}} \simeq \mathbf{0}
\end{aligned}
$$

\subsubsection{The velocity $\boldsymbol{u}$, electric field $\boldsymbol{E}$ and mass flux $\boldsymbol{m}$}

Since $\partial_{t} \boldsymbol{b}^{*}=-\boldsymbol{\nabla} \times \boldsymbol{E}^{*}\left(\boldsymbol{\nabla} \cdot \boldsymbol{b}^{*}=0\right), \partial_{t} \rho^{*}=-\boldsymbol{\nabla} \cdot \boldsymbol{m}^{*}$ (see (1a,d), (2a)) and $\partial_{t} \boldsymbol{b}=-\boldsymbol{\nabla} \times \boldsymbol{E}$ $(\boldsymbol{\nabla} \cdot \boldsymbol{b}=0), \partial_{t} \rho=-\boldsymbol{\nabla} \cdot \boldsymbol{m}($ see $(83 \mathrm{a}, \mathrm{c}, \mathrm{d}))$, we have

$$
\begin{aligned}
\partial_{t} \widetilde{\boldsymbol{b}}=-\nabla \times \widetilde{\boldsymbol{E}} & (\nabla \cdot \widetilde{\boldsymbol{b}}=0) ; & \widetilde{\boldsymbol{b}} \equiv \boldsymbol{b}^{*}+\boldsymbol{b}, & \widetilde{\boldsymbol{E}} \equiv \boldsymbol{E}^{*}+\boldsymbol{E}, \\
\partial_{t} \widetilde{\rho}=-\nabla \cdot \widetilde{\boldsymbol{m}} ; & & \widetilde{\rho} \equiv \rho^{*}+\rho, & \widetilde{\boldsymbol{m}} \equiv \boldsymbol{m}^{*}+\boldsymbol{m} .
\end{aligned}
$$

A word of caution: whereas $\boldsymbol{u}, \boldsymbol{m}, \boldsymbol{E}$ have the Lie derivatives (99c,d,e) respectively, in view

\footnotetext{
${ }^{1}$ If the covariant vector has weight $\mathrm{w}=-1$, the Lie derivative would be $\mathrm{L}_{\boldsymbol{\eta}} \boldsymbol{\mathcal { T }} \equiv\langle\boldsymbol{\eta}, \boldsymbol{T}\rangle \equiv\{\boldsymbol{\eta}, \boldsymbol{T}\}+(\boldsymbol{\nabla} \cdot \boldsymbol{\eta}) \boldsymbol{T}$. For example, Soward and Roberts (2008) encountered this Lie derivative in their eq. $(2.25 b): \boldsymbol{v} \mapsto \boldsymbol{\eta}, \rho \boldsymbol{v} \mapsto \rho \boldsymbol{V}=\mathcal{T}$.
} 
of their definitions $(65 a, b),(84 a-f)$, they have the Taylor series expansions

$$
\begin{aligned}
\boldsymbol{u}(\boldsymbol{x}, t) & =\left(\exp \mathrm{L}_{\boldsymbol{\eta}}\right) \boldsymbol{u}^{*}-\boldsymbol{w}, & \boldsymbol{u}^{*}(\boldsymbol{x}, t) & =\left(\exp \left(-\mathrm{L}_{\boldsymbol{\eta}}\right)\right) \boldsymbol{u}+\boldsymbol{w}^{*}, \\
\boldsymbol{E}(\boldsymbol{x}, t) & =\left(\exp \mathrm{L}_{\boldsymbol{\eta}}\right)\left(\boldsymbol{E}^{*}+\boldsymbol{w}^{*} \times \boldsymbol{b}^{*}\right), & \boldsymbol{E}^{*}(\boldsymbol{x}, t) & =\left(\exp \left(-\mathrm{L}_{\boldsymbol{\eta}}\right)\right)(\boldsymbol{E}-\boldsymbol{w} \times \boldsymbol{b}), \\
\boldsymbol{m}(\boldsymbol{x}, t) & =\left(\exp \mathrm{L}_{\boldsymbol{\eta}}\right)\left(\boldsymbol{m}^{*}-\rho^{*} \boldsymbol{w}^{*}\right), & \boldsymbol{m}^{*}(\boldsymbol{x}, t) & =\left(\exp \left(-\mathrm{L}_{\boldsymbol{\eta}}\right)\right)(\boldsymbol{m}+\rho \boldsymbol{w})
\end{aligned}
$$

instead of $(98 \mathrm{a}, \mathrm{b})$. Aided by $(103 \mathrm{c}, \mathrm{d})$ the differences of $(105 \mathrm{a}, \mathrm{b}),(105 \mathrm{c}, \mathrm{d}),(105 \mathrm{e}, \mathrm{f})$ yield

$$
\begin{aligned}
\boldsymbol{u}-\boldsymbol{u}^{*} & \approx-\partial_{t} \boldsymbol{\eta}+\frac{1}{2}\left[\boldsymbol{\eta},\left(\boldsymbol{u}+\boldsymbol{u}^{*}\right)\right], \\
2\left(\boldsymbol{E}-\boldsymbol{E}^{*}\right) & \approx\left(\partial_{t} \boldsymbol{\eta}\right) \times \widetilde{\boldsymbol{b}}+\{\boldsymbol{\eta}, \widetilde{\boldsymbol{E}}\}=\partial_{t}(\boldsymbol{\eta} \times \widetilde{\boldsymbol{b}})+\nabla(\boldsymbol{\eta} \cdot \widetilde{\boldsymbol{E}}), \\
2\left(\boldsymbol{m}-\boldsymbol{m}^{*}\right) & \approx-\widetilde{\rho} \partial_{t} \boldsymbol{\eta}+\llbracket \boldsymbol{\eta}, \widetilde{\boldsymbol{m}} \rrbracket=-\partial_{t}(\widetilde{\rho} \boldsymbol{\eta})-\nabla \times(\boldsymbol{\eta} \times \widetilde{\boldsymbol{m}})
\end{aligned}
$$

respectively, where in determining the final expressions in (106b,c) we have used (104a,d) and $(10 \mathrm{c}, \mathrm{b})$.

\section{$3.2 \quad$ Mean and fluctuating parts}

Our key assumption is that $\boldsymbol{u}$ has no fluctuating part $\left(\boldsymbol{u}^{\prime}=\mathbf{0}\right)$. It is then consistent with the HEL continuity equations $(83 \mathrm{~d}, \mathrm{c})$ to assume that $\rho$ and $\boldsymbol{m}$ have no fluctuating part $\left(\rho^{\prime}=0\right.$, $\boldsymbol{m}^{\prime}=\mathbf{0}$ ) either. In addition, we make the essential glm-ETHEL-postulate (see section 1.5) that $\boldsymbol{\eta}$ is purely fluctuating $\left(\boldsymbol{\eta}=\boldsymbol{\eta}^{\prime}\right)$ :

$$
\boldsymbol{u}=\overline{\boldsymbol{u}}, \quad \rho=\bar{\rho}, \quad \boldsymbol{m}=\overline{\boldsymbol{m}}, \quad \overline{\boldsymbol{\eta}}=\mathbf{0}
$$

implying

$$
\widetilde{\rho}^{\prime}=\rho^{* \prime}, \quad \widetilde{\boldsymbol{m}}^{\prime}=\boldsymbol{m}^{* \prime}
$$

When our tensors $\mathcal{T}$ have mean and fluctuating parts

$$
\mathcal{T}=\overline{\mathcal{T}}+\mathcal{T}^{\prime}
$$

we have

$$
\overline{\widetilde{\mathcal{T}}}=\overline{\mathcal{T}^{*}}+\overline{\mathcal{T}}, \quad \quad \widetilde{\mathcal{T}}^{\prime}=\mathcal{T}^{* \prime}+\mathcal{T}^{\prime}
$$

Generally the fluctuations are small: $\mathcal{T}^{\prime}=\mathrm{O}(\eta|\overline{\mathcal{T}}|), \mathcal{T}^{* \prime}=\mathrm{O}\left(\eta\left|\overline{\mathcal{T}^{*}}\right|\right)$ and $\overline{\mathcal{T}} \simeq \overline{\mathcal{T}^{*}}$. However, the fluctuating parts of the electromagnetic and thermal variables, $\boldsymbol{b}, \boldsymbol{E}$ and $\theta$, that originate from diffusion are even smaller:

$$
\boldsymbol{b}^{\prime}=\mathrm{O}(\mathfrak{K} \eta|\overline{\boldsymbol{b}}|), \quad \boldsymbol{E}^{\prime}=\mathrm{O}(\mathfrak{K} \eta|\overline{\boldsymbol{E}}|), \quad \theta^{\prime}=\mathrm{O}(\kappa \eta|\bar{\theta}|) .
$$

The mean and fluctuating parts of $(98 \mathrm{c})$ with $\mathcal{T}^{*}=\boldsymbol{b}^{*}, \rho^{*}, \theta^{*}$, having respective Lie derivatives (100a), (99b,a), determine with the help of $(108 \mathrm{a}, \mathrm{b})$ the ETHEL-representations

$$
\begin{array}{lll}
\overline{\boldsymbol{b}^{*}} \approx \overline{\boldsymbol{b}}+\frac{1}{2} \boldsymbol{\nabla} \times\left(\overline{\boldsymbol{\eta} \times \widetilde{\boldsymbol{b}}^{\prime}}\right), & \boldsymbol{b}^{* \prime} \bumpeq \boldsymbol{b}^{\prime}+\boldsymbol{\nabla} \times(\boldsymbol{\eta} \times \overline{\boldsymbol{b}}), \\
\overline{\rho^{*}} \approx \rho-\frac{1}{2} \boldsymbol{\nabla} \cdot\left(\overline{\rho^{* \prime} \boldsymbol{\eta}}\right), & \rho^{* \prime} \simeq-\nabla \cdot(\rho \boldsymbol{\eta}), \\
\overline{\theta^{*}} \bumpeq \bar{\theta}-\frac{1}{2} \overline{\boldsymbol{\eta} \cdot \boldsymbol{\nabla} \widetilde{\theta}^{\prime}}, & \theta^{* \prime} \bumpeq \theta^{\prime}-\boldsymbol{\eta} \cdot \boldsymbol{\nabla} \bar{\theta}, \\
\overline{q^{*}} \approx \bar{q}-\frac{1}{2} \overline{\boldsymbol{\eta} \cdot \boldsymbol{\nabla} \widetilde{q}^{\prime}}, & q^{* \prime} \bumpeq q^{\prime}-\boldsymbol{\eta} \cdot \boldsymbol{\nabla} \bar{q},
\end{array}
$$


while those for $\boldsymbol{u}^{*}, \boldsymbol{E}^{*}, \boldsymbol{m}^{*}$ obtained from (106a-c) are

$$
\begin{aligned}
& \overline{\boldsymbol{u}^{*}} \approx \boldsymbol{u}-\frac{1}{2} \overline{\left[\boldsymbol{\eta}, \boldsymbol{u}^{* \prime}\right]}, \\
& \boldsymbol{u}^{* \prime} \simeq \partial_{t} \boldsymbol{\eta}-[\boldsymbol{\eta}, \boldsymbol{u}] \\
& \overline{\boldsymbol{E}^{*}} \approx \overline{\boldsymbol{E}}-\frac{1}{2} \partial_{t}\left(\overline{\boldsymbol{\eta} \times \widetilde{\boldsymbol{b}}^{\prime}}\right)-\frac{1}{2} \boldsymbol{\nabla}\left(\overline{\boldsymbol{\eta} \cdot \widetilde{\boldsymbol{E}}^{\prime}}\right), \quad \boldsymbol{E}^{* \prime} \bumpeq \boldsymbol{E}^{\prime}-\partial_{t}(\boldsymbol{\eta} \times \overline{\boldsymbol{b}})-\nabla(\boldsymbol{\eta} \cdot \overline{\boldsymbol{E}}), \\
& \overline{\boldsymbol{m}^{*}} \approx \boldsymbol{m}+\frac{1}{2} \partial_{t}\left(\overline{\rho^{* \prime} \boldsymbol{\eta}}\right)+\frac{1}{2} \boldsymbol{\nabla} \times\left(\overline{\boldsymbol{\eta} \times \boldsymbol{m}^{* \prime}}\right), \quad \boldsymbol{m}^{* \prime} \simeq \partial_{t}\left(\overline{\rho^{*}} \boldsymbol{\eta}\right)+\nabla \times\left(\boldsymbol{\eta} \times \overline{\boldsymbol{m}^{*}}\right) .
\end{aligned}
$$

In $(110 \mathrm{~b}, \mathrm{f})$ and $(111 \mathrm{~d}$ ), we have used the $\bumpeq$ notation (see (15a)) to accommodate the higher order accuracy described by (109a-c) for the fluctuations. This is significant because it provides the higher order accuracy $\mathrm{O}\left(\mathfrak{K} \eta^{2} \overline{\boldsymbol{b}}\right)$ for $\overline{\boldsymbol{b}}^{*}$ in $(110 \mathrm{a}), \mathrm{O}\left(\mathfrak{K} \eta^{2} \overline{\boldsymbol{E}}\right)$ for $\overline{\boldsymbol{E}^{*}}$ in $(111 \mathrm{c})$ and $\mathrm{O}\left(\kappa \eta^{2} \bar{\theta}\right)$ for $\overline{\theta^{*}}$ in $(110 \mathrm{e}$ ), which we indicate by the $\approx$ notation (see $(15 \mathrm{~b})$ ). This improved accuracy is lost when $\boldsymbol{b}^{\prime}, \boldsymbol{E}^{\prime}$ and $\theta^{\prime}$ are neglected.

We compare the expansion (106a) of $\boldsymbol{u}-\boldsymbol{u}^{*}$ with the Frieman Rotenburg representation $\boldsymbol{u}^{*}-\overline{\boldsymbol{u}^{*}}=\partial_{t} \boldsymbol{\zeta}-\left[\boldsymbol{\zeta}, \overline{\boldsymbol{u}^{*}}\right]$ of section 1.1 in which $\overline{\boldsymbol{\zeta}}=\mathbf{0}$ (see $(9 \mathrm{a}, \mathrm{b})$ ). Their sum determines

$$
\left(\overline{\boldsymbol{u}^{*}}-\boldsymbol{u}\right)-\left[\boldsymbol{\eta},\left(\overline{\boldsymbol{u}^{*}}-\boldsymbol{u}\right)\right]+\partial_{t}(\boldsymbol{\zeta}-\boldsymbol{\eta})-\left[(\boldsymbol{\zeta}-\boldsymbol{\eta}), \overline{\boldsymbol{u}^{*}}\right] \approx \frac{1}{2}\left[\boldsymbol{\eta},\left(\boldsymbol{u}-\boldsymbol{u}^{*}\right)\right],
$$

the fluctuating part of which yields

$$
\partial_{t}(\boldsymbol{\zeta}-\boldsymbol{\eta})-\left[(\boldsymbol{\zeta}-\boldsymbol{\eta}), \overline{\boldsymbol{u}^{*}}\right] \approx-\frac{1}{2}\left[\boldsymbol{\eta}, \boldsymbol{u}^{* \prime}\right]^{\prime},
$$

where the prime on the Lie derivative denotes the fluctuating part. For given $\boldsymbol{u}$ and $\boldsymbol{\eta}$, the system (111a,b) and (112b) should be regarded as a set of equations that determine $\overline{\boldsymbol{u}^{*}}$ and $\boldsymbol{\zeta}$. For small $\boldsymbol{\eta}$, they provide the estimates

$$
\overline{\boldsymbol{u}^{*}} \simeq \boldsymbol{u}, \quad \boldsymbol{\zeta} \simeq \boldsymbol{\eta}, \quad \text { also } \quad \boldsymbol{\xi} \simeq \boldsymbol{\eta}
$$

(see (13a) and (95a)). Strictly, (112b) provides an evolution equation for the difference $\boldsymbol{\zeta}-\boldsymbol{\eta}$.

On replacing $\boldsymbol{\eta}$ by $\boldsymbol{\zeta}$, the above expressions (110a-h) and (111a-f) agree with our effectivevariable results of section 1 , all correct to $\mathrm{O}\left(\eta^{2}\right)$. So henceforth we make the exact identification

$$
\mathcal{T}^{\dagger} \equiv \mathcal{T}
$$

for all our variables $\mathcal{T}=\boldsymbol{u}, \boldsymbol{b}, \rho, \theta, q, \boldsymbol{E}$ and $\boldsymbol{m}$. In this way the ETHEL-development of HEL formalises the former ad hoc effective-variable approach and moreover provides a method that generates relations to any order of desired accuracy. That, only $\mathrm{O}\left(\eta^{2}\right)$ agreement has been achieved, is a reflection of the fact that $\boldsymbol{\eta} \neq \boldsymbol{\zeta}$ (see (113b)). At higher orders, the small differences between $\boldsymbol{\eta}$ and $\boldsymbol{\zeta}$ determined by (112b) need to be considered, a matter upon which we expand in Appendix G.

\subsection{The covariant electric current $\sigma J$ and thermal diffusion term $\kappa\left(\Delta \theta^{*}\right)^{L}$}

We consider the HEL-forms $\boldsymbol{J}$ and $\kappa\left(\Delta \theta^{*}\right)^{L}$ (see (81b) and (75e)) needed in the transformed magnetic induction and thermal diffusion equations $(127 \mathrm{~b}, \mathrm{c})$ below. As they appear in the small diffusive terms of those equations, we neglect the diffusive HEL-fluctuations:

$$
\boldsymbol{b}^{\prime} \simeq \mathbf{0}, \quad \theta^{\prime} \simeq 0,
$$

which means that contributions $\mathrm{O}\left(R m^{-1} \eta|\overline{\boldsymbol{b}}|\right)$ and $\mathrm{O}\left(P e^{-1} \eta|\bar{\theta}|\right)$ are ignored, as explained below (13a-c).

For $\mathcal{T}=\boldsymbol{J}$ with Lie derivative (99e), the mean an fluctuating parts of (98c) determine

$$
\overline{\boldsymbol{J}} \approx \overline{\boldsymbol{J}^{*}}+\frac{1}{2} \overline{\left\{\boldsymbol{\eta}, \widetilde{\boldsymbol{J}}^{\prime}\right\}}, \quad \begin{cases}\boldsymbol{J}^{\prime} \simeq \boldsymbol{J}^{* \prime}+\left\{\boldsymbol{\eta}, \overline{\boldsymbol{J}^{*}}\right\}, & \overline{\boldsymbol{J}^{*}}=\mathfrak{K} \boldsymbol{\nabla} \times \overline{\boldsymbol{b}^{*},} \\ \widetilde{\boldsymbol{J}}^{\prime}=\left(\boldsymbol{J}^{* \prime}+\boldsymbol{J}^{\prime}\right), & \boldsymbol{J}^{* \prime}=\mathfrak{K} \boldsymbol{\nabla} \times \boldsymbol{b}^{* \prime} .\end{cases}
$$


The mean and fluctuating parts of the electric field $\boldsymbol{E}(86 \mathrm{a})$ are thus

$$
\overline{\boldsymbol{E}} \approx-\boldsymbol{u} \times \overline{\boldsymbol{b}}+\overline{\boldsymbol{J}}, \quad\left\{\begin{array}{l}
\boldsymbol{E}^{\prime} \bumpeq-\boldsymbol{u} \times \boldsymbol{b}^{\prime}+\boldsymbol{J}^{\prime}, \\
\widetilde{\boldsymbol{E}}^{\prime} \bumpeq-\overline{\boldsymbol{u}^{*}} \times \widetilde{\boldsymbol{b}}^{\prime}-\boldsymbol{u}^{* \prime} \times \overline{\boldsymbol{b}^{*}}+\widetilde{\boldsymbol{J}}^{\prime},
\end{array}\right.
$$

in which we have used $\boldsymbol{E}^{* \prime} \bumpeq-\overline{\boldsymbol{u}^{*}} \times \boldsymbol{b}^{* \prime}-\boldsymbol{u}^{* \prime} \times \overline{\boldsymbol{b}^{*}}+\boldsymbol{J}^{* \prime}$ (see (31a)) and noted that $\boldsymbol{u} \simeq \overline{\boldsymbol{u}^{*}}$. In view of the frozen field approximation (115a), the mean and fluctuating parts of the magnetic field, given by (110a,b), are $\overline{\boldsymbol{b}^{*}} \approx \overline{\boldsymbol{b}}+\frac{1}{2} \boldsymbol{\nabla} \times \overline{\left(\boldsymbol{\eta} \times \boldsymbol{b}^{* \prime}\right)}$, where $\boldsymbol{b}^{* \prime} \simeq \boldsymbol{\nabla} \times(\boldsymbol{\eta} \times \overline{\boldsymbol{b}})$. On substituting these values into (116c), the formulae $(116 \mathrm{a}, \mathrm{b})$, we obtain

$$
\begin{aligned}
\mathfrak{K}^{-1} \mathcal{E} \equiv-\mathbf{J}= & -\mathfrak{K}^{-1} \overline{\boldsymbol{J}}+\boldsymbol{\nabla} \times \overline{\boldsymbol{b}} \\
\approx & \frac{1}{2} \Delta\left(\overline{\boldsymbol{\eta} \times \boldsymbol{b}^{* \prime}}\right)-\overline{\boldsymbol{\eta} \times \Delta \boldsymbol{b}^{* \prime}}+\frac{1}{2} \overline{\boldsymbol{\eta} \times(\boldsymbol{\nabla} \times(\boldsymbol{\eta} \times \Delta \overline{\boldsymbol{b}}))} \\
& -\nabla\left(\frac{1}{2} \boldsymbol{\nabla} \cdot\left(\overline{\boldsymbol{\eta} \times \boldsymbol{b}^{* \prime}}\right)+\overline{\boldsymbol{\eta} \cdot\left(\boldsymbol{\nabla} \times \boldsymbol{b}^{* \prime}\right)}+\frac{1}{2} \overline{\boldsymbol{\eta} \cdot \boldsymbol{\nabla}(\boldsymbol{\eta} \cdot(\boldsymbol{\nabla} \times \overline{\boldsymbol{b}}))}\right) .
\end{aligned}
$$

Here we introduce $\mathfrak{K} \mathbf{J} \equiv-\mathcal{E}$, for consistency with our notation $\mathfrak{K} \mathbf{J}^{\dagger} \equiv-\mathcal{E}^{\dagger}$ in (43b) and again emphasise its origin in magnetic diffusion.

Though (118) makes the connection with the result (43c) for effective-variables, the elimination of the terms in it involving the second and third derivatives to reach the form

$$
-\mathbf{J} \doteq \overline{\boldsymbol{\alpha}} \cdot \overline{\boldsymbol{b}}-((\overline{\boldsymbol{g}}-\mathbf{I}) \cdot \nabla) \times \overline{\boldsymbol{b}},
$$

namely the average of $\mathfrak{K}^{-1} \boldsymbol{J}$ given by (88), is far from straightforward. To construct this alternative form, it only remains to determine $\overline{\boldsymbol{g}}-\mathbf{I}$ and $\overline{\boldsymbol{\alpha}}$. According to (A.5) the unweighted contravariant tensor $\boldsymbol{g}$ (see (68)) has Lie derivative

$$
\mathrm{L}_{\boldsymbol{\eta}} \boldsymbol{g} \equiv(\boldsymbol{\eta} \cdot \boldsymbol{\nabla}) \boldsymbol{g}-\boldsymbol{g} \cdot(\nabla \boldsymbol{\eta})-(\boldsymbol{\nabla} \boldsymbol{\eta})^{\mathrm{T}} \cdot \boldsymbol{g}
$$

with the property

$$
\mathrm{L}_{\boldsymbol{\eta}} \mathbf{I}=-\mathbf{e}, \quad \text { where } \quad \mathbf{e} \equiv(\boldsymbol{\nabla} \boldsymbol{\eta})+(\boldsymbol{\nabla} \boldsymbol{\eta})^{\mathrm{T}} .
$$

The corresponding Taylor series expansion of $\boldsymbol{g}$ is is

$$
\boldsymbol{g}=\exp \left(\mathrm{L}_{\boldsymbol{\eta}}\right) \mathbf{I}=\mathbf{I}-\mathbf{e}-\frac{1}{2} \mathrm{~L}_{\boldsymbol{\eta}} \mathbf{e}-\cdots,
$$

Its mean part determines

$$
\overline{\boldsymbol{g}}-\mathbf{I} \approx-\frac{1}{2} \overline{\mathrm{L}_{\boldsymbol{\eta}} \mathbf{e}}=-\frac{1}{2} \overline{(\boldsymbol{\eta} \cdot \boldsymbol{\nabla}) \mathbf{e}}+\frac{1}{2} \overline{\mathbf{e} \cdot(\boldsymbol{\nabla} \boldsymbol{\eta})}+\frac{1}{2} \overline{(\boldsymbol{\nabla} \boldsymbol{\eta})^{\mathrm{T}} \cdot \mathbf{e}} .
$$

This result is needed to determine the anti-symmetric part of the mean of (90a), namely

$$
\overline{\alpha_{i j}}=\frac{1}{2}\left(\overline{\alpha_{i j}}+\overline{\alpha_{j i}}\right)+\frac{1}{2} \epsilon_{i j k} \nabla_{l} \overline{g_{l k}}, \quad \text { where } \quad \breve{\alpha}_{i j}=\left(\nabla_{m}^{L} x_{k}\right) \epsilon_{k l i} \nabla_{j}\left(\nabla_{m}^{L} x_{l}\right) \text {. }
$$

Recalling that $\boldsymbol{\nabla}^{L} \boldsymbol{x} \approx \mathbf{I}-\boldsymbol{\nabla} \boldsymbol{\eta}-\frac{1}{2}[\boldsymbol{\eta}, \boldsymbol{\nabla} \boldsymbol{\eta}]$ (see (96c)), we may write

$$
\overline{\breve{\alpha}_{i j}} \approx \nabla_{j}\left(\overline{\nabla^{L} \times \boldsymbol{x}}\right)_{i}+\overline{\left(\nabla_{m}^{L} \eta_{k}\right) \epsilon_{k l i} \nabla_{j}\left(\nabla_{m}^{L} \eta_{l}\right)} .
$$

Here, since $\epsilon_{i k l} \overline{\nabla_{k}^{L} x_{l}} \approx-\frac{1}{2} \epsilon_{i k l}\left(\overline{\left(\eta_{m} \nabla_{m}\right)\left(\nabla_{k} \eta_{l}\right)}-\overline{\left(\nabla_{k} \eta_{m}\right)\left(\nabla_{m} \eta_{l}\right)}\right)$, use of the identity (B.3) determines

$$
\overline{\boldsymbol{\nabla}^{L} \times \boldsymbol{x}} \approx-\frac{1}{2}(\{\boldsymbol{\eta},(\boldsymbol{\nabla} \times \boldsymbol{\eta})\}-(\boldsymbol{\nabla} \cdot \boldsymbol{\eta})(\boldsymbol{\nabla} \times \boldsymbol{\eta})) .
$$

Together (120c), (121b) and (122a-d) determine the coefficients $\overline{\boldsymbol{g}}-\mathbf{I}$ and $\overline{\boldsymbol{\alpha}}$ in the relation (119) for $\mathbf{J}$ correct to $\mathrm{O}\left(\eta^{2}\right)$. As mentioned above the direct reduction of (118) to (119), namely the effective-variable form (43c), with these coefficient values is formidable. The essential point here is that the ETHEL-approach, which bypasses (43c), is far preferable. 
The thermal diffusion term proportional to $\left(\Delta \theta^{*}\right)^{L}$ can be treated in a similar way. On averaging the expression $\left(\Delta \theta^{*}\right)^{L}=\mathcal{J}^{-1} \boldsymbol{\nabla} \cdot(\mathcal{J} \boldsymbol{g} \cdot \boldsymbol{\nabla} \theta)$ given by $(75 \mathrm{e})$, we obtain

$$
\mathrm{H} \equiv \overline{\left(\Delta \theta^{*}\right)^{L}}-\Delta \bar{\theta} \doteq \overline{\mathcal{J}^{-1}(\boldsymbol{\nabla J}) \boldsymbol{g}} \cdot \boldsymbol{\nabla} \bar{\theta}+\boldsymbol{\nabla} \cdot((\overline{\boldsymbol{g}}-\mathbf{I}) \cdot \boldsymbol{\nabla} \bar{\theta})
$$

where

$$
\overline{\mathcal{J}^{-1}(\boldsymbol{\nabla} \mathcal{J}) \boldsymbol{g}} \approx \frac{1}{2} \boldsymbol{\nabla}(\overline{\boldsymbol{\eta} \cdot \boldsymbol{\nabla}(\boldsymbol{\nabla} \cdot \boldsymbol{\eta})})-\overline{\mathbf{e} \cdot \boldsymbol{\nabla}(\boldsymbol{\nabla} \cdot \boldsymbol{\eta})} .
$$

In summary, on the basis that $\boldsymbol{\zeta} \simeq \boldsymbol{\eta}$, we have $\mathbf{e}^{\ddagger} \simeq \mathbf{e}$ and $\overline{\boldsymbol{g}^{\ddagger}} \approx \overline{\boldsymbol{g}}$ (since $\overline{\boldsymbol{\eta}}=\overline{\boldsymbol{\zeta}}=\mathbf{0}$ ), with the consequence that we may demand $\mathbf{J}^{\dagger} \equiv \mathbf{J}$ (cf. (43c) to (118)) and $\mathbf{H}^{\dagger} \equiv \mathbf{H}$ (cf. (45c) to $(123 a, b))$, as advocated by (114). Furthermore, the use of $\doteq$ (defined by (14)) in (119) and (123a) flags up the fact that, on averaging the exact formulae (88) and (75e) for $\mathfrak{K}^{-1} \boldsymbol{J}$ and $\left(\Delta \theta^{*}\right)^{L}$, we have neglected averages like $\overline{\boldsymbol{g}^{\prime} \cdot \boldsymbol{\nabla} \boldsymbol{b}^{\prime}}$ and $\overline{\boldsymbol{g}^{\prime} \cdot \boldsymbol{\nabla} \theta^{\prime}}$ involving $\boldsymbol{b}^{\prime}=\mathrm{O}\left(R m^{-1} \eta \overline{\boldsymbol{b}}\right)$ and $\theta^{\prime}=\mathrm{O}\left(P e^{-1} \eta \bar{\theta}\right)$. Since $\mathbf{J}=\mathrm{O}\left(\eta^{2} \overline{\boldsymbol{b}}\right)$ and $\mathbf{H}=\mathrm{O}\left(\eta^{2} \bar{\theta}\right)$, the neglected averages $\mathrm{O}\left(R m^{-1} \eta^{2} \overline{\boldsymbol{b}}\right)$ and $\mathrm{O}\left(P e^{-1} \eta^{2} \bar{\theta}\right)$ are respectively an order of magnitude $\mathrm{O}\left(R m^{-1}\right)$ and $\mathrm{O}\left(P e^{-1}\right)$ smaller. This feature sums up succinctly the high order accuracy of the GLM (equivalently) glm approach.

\section{The governing equations}

We consider a Boussinesq fluid, density $\rho^{*}=\rho_{0}$, for which density variations are ignored except where they give rise to buoyancy forces. At position P: $\boldsymbol{x}$ it moves with velocity $\boldsymbol{u}^{*}(\boldsymbol{x}, t)$ and possesses momentum per unit mass $\boldsymbol{V}^{*}(\boldsymbol{x}, t)\left(=\boldsymbol{u}^{*}(\boldsymbol{x}, t)\right)$ in a frame rotating with angular velocity $\boldsymbol{\Omega}$. The fluid is inviscid, electrically conducting and permeated by a magnetic field $\boldsymbol{b}^{*}(\boldsymbol{x}, t)$. Though, for simplicity, we have omitted viscosity, it may be readily reinstated when necessary. The equations

$$
\begin{gathered}
\partial_{t} \boldsymbol{V}^{*}+\left(\boldsymbol{\nabla} \times \boldsymbol{V}^{*}\right) \times \boldsymbol{u}^{*}+\mathcal{C}^{*}=-\nabla \Pi_{+}^{*}+\rho_{0}^{-1} \boldsymbol{j}^{*} \times \boldsymbol{b}^{*}+\boldsymbol{F}^{*}, \\
\partial_{t} \boldsymbol{b}^{*}=\boldsymbol{\nabla} \times\left(\boldsymbol{u}^{*} \times \boldsymbol{b}^{*}-\boldsymbol{J}^{*}\right), \quad \boldsymbol{J}^{*}=\mathfrak{K} \boldsymbol{\nabla} \times \boldsymbol{b}^{*}, \\
\partial_{t} \theta^{*}+\boldsymbol{u}^{*} \cdot \boldsymbol{\nabla} \theta^{*}=\kappa \Delta \theta^{*}+q^{*}
\end{gathered}
$$

governing momentum, magnetic induction (see (1a-g)) and heat conduction (see (3a)) respectively, are supplemented by the solenoidal conditions

$$
\boldsymbol{\nabla} \cdot \boldsymbol{u}^{*}=0, \quad \boldsymbol{\nabla} \cdot \boldsymbol{b}^{*}=0
$$

(see $(2 \mathrm{a}, \mathrm{b}),(1 \mathrm{~d}))$. In the momentum equation (124a) the Coriolis acceleration $\mathcal{C}^{*}$, the modified pressure $\Pi_{+}^{*}$, the electric current $\boldsymbol{j}^{*}$ and buoyancy force $\rho_{0} \boldsymbol{F}^{*}$ are given by

$$
\mathcal{C}^{*} \equiv 2 \boldsymbol{\Omega} \times \boldsymbol{u}^{*}, \quad \Pi_{+}^{*} \equiv\left(p^{*} / \rho_{0}\right)+\frac{1}{2}\left|\boldsymbol{u}^{*}\right|^{2}, \quad \boldsymbol{F}^{*}=-\theta^{*} \boldsymbol{G}^{*}
$$

respectively. Remember that the electric current is $\boldsymbol{j}^{*}=\boldsymbol{\nabla} \times \boldsymbol{H}^{*}$ with $\boldsymbol{H}^{*}=\mu^{-1} \boldsymbol{b}^{*}$ (see $(1 \mathrm{c}, \mathrm{e}))$. We have assumed that the excess density is $-\rho_{0} \theta^{*}$, i.e. of thermal origin, so that the buoyancy force is $-\rho_{0} \theta^{*} G^{*}$, where $G^{*}$ is the gravitational acceleration; the temperature $\theta^{*}$ is governed by the heat conduction equation (124d).

\subsection{The Hybrid Euler-Lagrange (HEL) formulation}

As in sections 2 and 3, we consider the nature of the governing equations (124) - (126) at the point $\mathrm{P}^{L}: \boldsymbol{x}^{L}(\boldsymbol{x}, t)$. Our HEL-strategy is to multiply the $j^{\text {th }}$ component of (124a) by $\nabla_{i} x_{j}^{L}$, the $j^{\text {th }}$ component of (124b) by $\mathcal{J} \nabla_{j}^{L} x_{i}$, but leaving (124c) as it is; symbolically we denote the three multiplications by $\left(\boldsymbol{\nabla} \boldsymbol{x}^{L}\right) \cdot(124 \mathrm{a})^{L}, \mathcal{J}(124 \mathrm{~b})^{L} \cdot\left(\boldsymbol{\nabla}^{L} \boldsymbol{x}\right)$ and $(124 \mathrm{c})^{L}$ respectively. The 
transformed equations are

$$
\begin{gathered}
\partial_{t}(\boldsymbol{V}+\boldsymbol{\mathcal { R }})+(2 \boldsymbol{\Omega}+\boldsymbol{\nabla} \times(\boldsymbol{V}+\boldsymbol{\mathcal { R }})) \times \boldsymbol{u}=-\boldsymbol{\nabla} \Pi_{T}+\rho^{-1} \boldsymbol{j} \times \boldsymbol{b}+\boldsymbol{F}, \\
\partial_{t} \boldsymbol{b}=\boldsymbol{\nabla} \times(\boldsymbol{u} \times \boldsymbol{b}-\boldsymbol{J}), \\
\mathrm{D}_{t} \theta=\kappa \boldsymbol{\nabla} \cdot(\boldsymbol{g} \cdot \boldsymbol{\nabla} \theta)+\mathcal{J}^{-1}(\boldsymbol{\nabla} \mathcal{J}) \boldsymbol{g} \cdot \boldsymbol{\nabla} \theta+q .
\end{gathered}
$$

The HEL magnetic induction equation (127b) with $\mathfrak{K}^{-1} \boldsymbol{J}=(\boldsymbol{g} \cdot \boldsymbol{\nabla}) \times \boldsymbol{b}-\boldsymbol{\alpha} \cdot \boldsymbol{b}$ (see (88)) follows from (80b), while the HEL heat conduction equation (127c) is simply (82d).

In the HEL equation of motion (127a), we recall that (61c) says that $\mathcal{J}^{-1} \rho=\rho_{0}=$ constant. It therefore follows from (73b) that the Lorentz force transforms as

$$
\rho^{-1} \boldsymbol{j} \times \boldsymbol{b}=\rho_{0}^{-1}\left(\boldsymbol{\nabla} \boldsymbol{x}^{L}\right) \cdot\left(\boldsymbol{j}^{*} \times \boldsymbol{b}^{*}\right)^{L} .
$$

We supplement the definitions (61)-(68) and (72b,c) with

$$
\boldsymbol{F}=\left(\boldsymbol{\nabla} \boldsymbol{x}^{L}\right) \cdot \boldsymbol{F}^{* L}=-\theta \boldsymbol{G}, \quad \boldsymbol{G}=\left(\boldsymbol{\nabla} \boldsymbol{x}^{L}\right) \cdot \boldsymbol{G}^{* L}, \quad \boldsymbol{\nabla} \Pi_{+}=\left(\boldsymbol{\nabla} \boldsymbol{x}^{L}\right) \cdot\left(\boldsymbol{\nabla} \Pi_{+}^{*}\right)^{L},(129 \mathrm{a}-\mathrm{c})
$$

in which $\Pi_{+}=\Pi_{+}^{* L}$, and introduce

$$
\Pi_{T} \equiv \Pi_{+}-\left(\partial_{t} \boldsymbol{\xi}\right) \cdot\left(\boldsymbol{V}^{* L}+\boldsymbol{\Omega} \times \boldsymbol{\xi}\right) .
$$

Aided with the result (79d) for the time derivative plus Lie derivative combination $\partial_{t}+\mathrm{L}_{\boldsymbol{u}^{*}}$, the term involving the rate of change of momentum transforms as follows:

$$
\begin{aligned}
\left(\boldsymbol{\nabla} \boldsymbol{x}^{L}\right) \cdot\left(\partial_{t} \boldsymbol{V}^{*}+\left(\boldsymbol{\nabla} \times \boldsymbol{V}^{*}\right) \times \boldsymbol{u}^{*}\right)^{L} & =\left(\boldsymbol{\nabla} \boldsymbol{x}^{L}\right) \cdot\left(\partial_{t} \boldsymbol{V}^{*}+\mathrm{L}_{\boldsymbol{u}^{*}} \boldsymbol{V}^{*}-\boldsymbol{\nabla}\left(\boldsymbol{u}^{*} \cdot \boldsymbol{V}^{*}\right)\right)^{L} \\
& =\partial_{t} \boldsymbol{V}+\mathrm{L}_{\boldsymbol{u}} \boldsymbol{V}-\boldsymbol{\nabla}\left(\boldsymbol{u}^{* L} \cdot \boldsymbol{V}^{* L}\right) \\
& =\partial_{t} \boldsymbol{V}+(\boldsymbol{\nabla} \times \boldsymbol{V}) \times \boldsymbol{u}-\boldsymbol{\nabla}\left(\boldsymbol{u}^{* L} \cdot \boldsymbol{V}^{* L}-\boldsymbol{u} \cdot \boldsymbol{V}\right) \\
& =\partial_{t} \boldsymbol{V}+(\boldsymbol{\nabla} \times \boldsymbol{V}) \times \boldsymbol{u}-\boldsymbol{\nabla}\left(\left(\partial_{t} \boldsymbol{\xi}\right) \cdot \boldsymbol{V}^{* L}\right),
\end{aligned}
$$

in which we have noted that $\boldsymbol{u} \cdot \boldsymbol{V}=\left(\boldsymbol{u}^{* L}-\boldsymbol{w}^{* L}\right) \cdot \boldsymbol{V}^{* L}$ and $\boldsymbol{w}^{* L}=\partial_{t} \boldsymbol{\xi}$ (see (65a,b) and $(59 \mathrm{~b}))$. On the introduction of

$$
\boldsymbol{\mathcal { R }} \equiv\left(\mathbf{I}+\boldsymbol{\nabla} \boldsymbol{x}^{L}\right) \cdot(\boldsymbol{\Omega} \times \boldsymbol{\xi})=\boldsymbol{\Omega} \times(2 \boldsymbol{\xi}+((\boldsymbol{\nabla} \cdot \boldsymbol{\xi}) \boldsymbol{\xi}-\boldsymbol{\xi} \cdot \boldsymbol{\nabla} \boldsymbol{\xi}))-(\boldsymbol{\Omega} \cdot \boldsymbol{\nabla} \boldsymbol{\xi}) \times \boldsymbol{\xi},
$$

whose far right-hand side is obtained from (E.8), we show in Appendix E that the Coriolis acceleration (126a) has the HEL-form

$$
\mathcal{C}=\left(\nabla \boldsymbol{x}^{L}\right) \cdot \mathcal{C}^{* L}=\partial_{t} \boldsymbol{R}+(2 \boldsymbol{\Omega}+\boldsymbol{\nabla} \times \boldsymbol{\mathcal { R }}) \times \boldsymbol{u}-\boldsymbol{\nabla}\left(\left(\partial_{t} \boldsymbol{\xi}\right) \cdot(\boldsymbol{\Omega} \times \boldsymbol{\xi})\right)
$$

(see (E.7)), in which we highlight the term $2 \boldsymbol{\Omega} \times \boldsymbol{u}$. The HEL mean value $\overline{\mathcal{C}}$ of (132) deserves comparison with the pioneering analysis in Appendix B of Andrews and McIntyre (1978a) particularly their (B.6) and (B.7), albeit that concerns evaluation of the means of $(196 \mathrm{a}, \mathrm{b})$ below under the assumption $\overline{\boldsymbol{\xi}}=\mathbf{0}$ (see (195a)) not made elsewhere in our paper.

The constant density assumption $\rho=\rho_{0}$ implies that the mass continuity equations $(83 \mathrm{~d}, \mathrm{e})$ and solenoidal conditions $(125 \mathrm{a}, \mathrm{b})$ become

$$
\partial_{t} \rho+\nabla \cdot(\rho \boldsymbol{u})=0, \quad \boldsymbol{\nabla} \cdot(\rho \boldsymbol{v})=0, \quad \boldsymbol{\nabla} \cdot \boldsymbol{b}=0,
$$

where $\boldsymbol{v}=\boldsymbol{u}+\boldsymbol{w}$, consistent with $\partial_{t} \mathcal{J}=\boldsymbol{\nabla} \cdot(\mathcal{J} \boldsymbol{w})$ (see (A.2d)).

\subsection{The Euler Transformed HEL-formulation with $\mathcal{J}=1$}

Since our flow is solenoidal, it is natural to suppose henceforth that the HEL-mapping $\boldsymbol{x} \mapsto \boldsymbol{x}^{L}$, that takes $\mathrm{P}$ to $\mathrm{P}^{L}$ is isochoric, i.e., the Jacobian $\mathcal{J}$ of the transformation is unity:

$$
\mathcal{J}=\left\|\nabla \boldsymbol{x}^{L}\right\|=1 \text {. }
$$


Furthermore, since $\rho=\mathcal{J} \rho_{0}=\rho_{0}$ is constant, (133a,b) simplify respectively to

$$
\boldsymbol{\nabla} \cdot \boldsymbol{u}=0, \quad \boldsymbol{\nabla} \cdot \boldsymbol{w}=0, \quad \text { while } \quad \boldsymbol{\nabla} \cdot \boldsymbol{\eta}=0
$$

consistent with (95b) when $\mathcal{J}=1$. This solenoidal feature of $\boldsymbol{\eta}$ is attractive, because the corresponding statement $\boldsymbol{\nabla} \cdot \boldsymbol{\xi}=0$ generally requires $\mathcal{J} \neq 1$.

We have already explained in section 3 how to derive the ETHEL-forms summarised in (99a-e). Though $\mathcal{C}$ is itself a covariant vector, we have expressed it in (132) in terms of $\mathcal{R}$ and even $\boldsymbol{\Omega}$, which have not been constructed by general tensor rules. For that reason we cannot use our Lie derivative Taylor series to determine the ETHEL-expansion of $\mathcal{R}$. Instead, we derive its ETHEL-form directly from (131) using the expansion (95a) for $\boldsymbol{\xi}$. Noting that $\boldsymbol{\nabla} \cdot \boldsymbol{\eta}=0$, the result is

$$
\mathcal{R} \approx 2 \boldsymbol{\Omega} \times \boldsymbol{\eta}-(\boldsymbol{\Omega} \cdot \boldsymbol{\nabla} \boldsymbol{\eta}) \times \boldsymbol{\eta},
$$

where terms $\mathrm{O}\left(\eta^{3}|\boldsymbol{\Omega}|\right)$ have been neglected.

\subsection{Zero mass flux boundary condition}

For fluid contained within an impermeable stationary boundary $\mathcal{S}$ with surface vector element $\mathrm{d} \boldsymbol{S}^{*}$ outwardly directed, we apply the boundary condition $\boldsymbol{u}^{*} \cdot \mathrm{d} \boldsymbol{S}^{*}=0$ everywhere on $\mathcal{S}$. We assume that each point $\mathrm{P}: \boldsymbol{x} \in \mathcal{S}$ is displaced to another point $\mathrm{P}^{L}: \boldsymbol{x}^{L}(\boldsymbol{x}, t)=\boldsymbol{x}+\boldsymbol{\xi}(\boldsymbol{x}, t) \in \mathcal{S}$. Thus any surface element $\mathrm{d} \boldsymbol{S}$ on $\mathcal{S}$ at $\mathrm{P}$ is mapped to another surface element $\mathrm{d} \boldsymbol{S}^{* L}$ on $\mathcal{S}$ at $\mathrm{P}^{L}$; more precisely the map $\mathcal{S} \mapsto \mathcal{S}$ is a bijection. As a consequence the flux relation (85a) implies that $(\boldsymbol{u}+\boldsymbol{w}) \cdot \mathrm{d} \boldsymbol{S}=\boldsymbol{u}^{* L} \cdot \mathrm{d} \boldsymbol{S}^{* L}=0$ on $\mathcal{S}$. Furthermore, we have $\partial_{t} \boldsymbol{x}^{L} \cdot \mathrm{d} \boldsymbol{S}^{* L}=0$ by construction which with (61a) and (85b) implies that $\boldsymbol{w} \cdot \mathrm{d} \boldsymbol{S}=\boldsymbol{w}^{* L} \cdot \mathrm{d} \boldsymbol{S}^{* L}=0$ on $\mathcal{S}$. Together our results for $\boldsymbol{u}^{* L}$ and $\boldsymbol{w}^{* L}$ show that $\boldsymbol{u} \cdot \mathrm{d} \boldsymbol{S}=0$ on $\mathcal{S}$. Since the vector field $\boldsymbol{\eta}$ is interpreted as a 'fictitious' instantaneous steady velocity field that realises the displacement $\boldsymbol{\xi}$, we naturally assume $\boldsymbol{\eta}$ to be tangent to the boundary $\mathcal{S}$. In this way we deduce the important impermeable boundary conditions

$$
\boldsymbol{u} \cdot \mathrm{d} \boldsymbol{S}=0, \quad \boldsymbol{\eta} \cdot \mathrm{d} \boldsymbol{S}=0 \quad \text { on } \mathcal{S} .
$$

\section{The Braginsky dynamo in HEL-form}

We describe the Braginsky (1964a,b) dynamo relative to cylindrical polar coordinates $(s, \phi, z)$ with respective unit vectors

$$
\mathbf{1}^{1}=\widehat{s}, \quad \mathbf{1}^{2}=\widehat{\phi}, \quad \mathbf{1}^{3}=\widehat{\boldsymbol{z}}
$$

chosen such that

$$
\Omega=\Omega \widehat{z} .
$$

Relative to these coordinates, we express vectors such as $\boldsymbol{\eta}$ and gradients by

$$
\boldsymbol{\eta}=\left(\eta_{s}, \eta_{\phi}, \eta_{z}\right), \quad \boldsymbol{\nabla}=\left(\frac{\partial}{\partial s}, \frac{1}{s} \frac{\partial}{\partial \phi}, \frac{\partial}{\partial z}\right) .
$$

We use the abbreviations $\partial_{s} \equiv \partial / \partial s, \partial_{\phi} \equiv \partial / \partial \phi, \partial_{z} \equiv \partial / \partial z$, where $\partial_{\phi}$ does not differentiate unit vectors. We depart here from Braginsky's notation, who employed $\partial_{1 \phi}$ when he held the unit vectors fixed. If we distinguish $\partial \boldsymbol{\eta} / \partial \phi$ from $\partial_{\phi} \boldsymbol{\eta}$, using the former to mean that the unit vectors are differentiated, we have

$$
\partial \boldsymbol{\eta} / \partial \phi=\partial_{\phi} \boldsymbol{\eta}+\widehat{\boldsymbol{z}} \times \boldsymbol{\eta}, \quad \text { where } \quad \partial_{\phi} \boldsymbol{\eta} \equiv\left(\partial_{\phi} \eta_{s}, \partial_{\phi} \eta_{\phi}, \partial_{\phi} \eta_{z}\right),
$$


as in (47a,b). We write $\eta^{1}=\mathbf{1}^{1} \cdot \boldsymbol{\eta}=\eta_{s}, \eta^{2}=\mathbf{1}^{2} \cdot \boldsymbol{\eta}=\eta_{\phi}$ and $\eta^{3}=\mathbf{1}^{3} \cdot \boldsymbol{\eta}=\eta_{z}$, and similarly $\nabla^{i}=\mathbf{1}^{i} \cdot \boldsymbol{\nabla}$, where $(i=1,2,3)$, in order to distinguish them from the Cartesian components $\eta_{i}=\mathbf{1}_{i} \cdot \boldsymbol{\eta}$ and $\nabla_{i}=\mathbf{1}_{i} \cdot \boldsymbol{\nabla}$. As an example, we note the following useful application of (140a,b):

$$
(\boldsymbol{u} \cdot \boldsymbol{\nabla} \boldsymbol{\eta})^{p}=(\boldsymbol{u} \cdot \boldsymbol{\nabla}) \eta^{p}+s^{-1} u_{\phi}(\widehat{\boldsymbol{z}} \times \boldsymbol{\eta})^{p} .
$$

To transform from polar to Cartesian coordinates and vice versa, we need

$$
\eta_{i}=l_{i}^{j} \eta^{j}, \quad \nabla_{i}=l_{i}^{j} \nabla^{j}, \quad l_{i}^{j}=\mathbf{1}_{i} \cdot \mathbf{1}^{j} .
$$

Here the direction cosines $l_{i}^{j}$ of the transformation from one orthogonal coordinate system to another have their usual properties, e.g.,

$$
\delta_{i j}=l_{i}^{p} l_{j}^{q} \delta^{p q}, \quad \epsilon_{i j k}=l_{i}^{p} l_{j}^{q} l_{k}^{r} \epsilon^{p q r},
$$

where $\delta^{p q}\left(\equiv \delta_{p q}\right), \epsilon^{p q r}\left(\equiv \epsilon_{p q r}\right)$ are written with superscripts to emphasise that they are linked to the cylindrical polar coordinates. Nevertheless, we need to be aware that $s \nabla^{2} \mathbf{1}^{i}=\epsilon^{3 i j} \mathbf{1}^{j}$, from which it follows that

$$
s \nabla^{i} l_{j}^{k}=\delta^{i 2} \epsilon^{3 k l} l_{j}^{l} .
$$

(Note that, in this section and Appendix F, $\nabla^{2}$ does not mean $\boldsymbol{\nabla} \cdot \boldsymbol{\nabla}$ for which we write $\Delta$ instead, see (3b)). A consequence of (144) is that

$$
(\boldsymbol{\nabla} \boldsymbol{\eta})_{i j} \equiv \nabla_{i} \eta_{j}=l_{i}^{p} l_{j}^{q}(\boldsymbol{\nabla} \boldsymbol{\eta})^{p q}
$$

where

$$
(\nabla \boldsymbol{\eta})^{p q}=\nabla^{p} \eta^{q}+s^{-1} \delta^{p 2} \epsilon^{3 r q} \eta^{r}
$$

e.g., the special case $p=2$ recovers (140a). The gradients of higher rank tensors may be dealt with in exactly the same way. So, for example, the gradient of any second rank tensor $\boldsymbol{g}$ is

$$
(\boldsymbol{\nabla} \boldsymbol{g})_{i j k} \equiv \nabla_{i} g_{j k}=l_{i}^{p} l_{j}^{q} l_{k}^{r}(\boldsymbol{\nabla} \boldsymbol{g})^{p q r},
$$

where

$$
(\boldsymbol{\nabla g})^{p q r}=\nabla^{p} g^{q r}+s^{-1} \delta^{p 2}\left(\epsilon^{3 s q} g^{s r}+\epsilon^{3 s r} g^{q s}\right) .
$$

We denote the $\phi$-average of a scalar such as $\theta$ by $\bar{\theta}$. In the case of vectors such a $\boldsymbol{\eta}$, we take the average of each of its cylindrical polar components, so that

$$
(\overline{\boldsymbol{\eta}})^{r} \equiv \overline{\eta^{r}}
$$

and in the same spirit, from (145b), we define

$$
(\bar{\nabla} \boldsymbol{\eta})^{p q} \equiv \nabla^{p} \overline{\eta^{q}}+s^{-1} \delta^{p 2} \epsilon^{3 r q} \overline{\eta^{r}}=(\boldsymbol{\nabla} \overline{\boldsymbol{\eta}})^{p q} .
$$

For the particular case $p=2$, the $\phi$-average of (140a) determines

$$
\overline{\partial \boldsymbol{\eta} / \partial \phi}=\widehat{\boldsymbol{z}} \times \overline{\boldsymbol{\eta}}, \quad \text { since } \quad \overline{\partial_{\phi} \boldsymbol{\eta}}=\partial_{\phi} \overline{\boldsymbol{\eta}}=\mathbf{0} .
$$

The identity $\overline{\nabla \boldsymbol{\eta}}=\boldsymbol{\nabla} \overline{\boldsymbol{\eta}}$ (see (147b)), says that the averaging operation commutes with the gradient. Some useful consequences are

$$
\begin{aligned}
\overline{\boldsymbol{\nabla} \cdot \boldsymbol{\eta}} & =\boldsymbol{\nabla} \cdot \overline{\boldsymbol{\eta}}=\boldsymbol{\nabla} \cdot \overline{\boldsymbol{\eta}_{\mathrm{m}}}, \\
\overline{\boldsymbol{\nabla} \times \boldsymbol{\eta}} & =\boldsymbol{\nabla} \times \overline{\boldsymbol{\eta}}=\boldsymbol{\nabla} \times \overline{\boldsymbol{\eta}_{\mathrm{m}}}+s^{-1} \boldsymbol{\nabla}\left(s \overline{\eta_{\phi}}\right) \times \widehat{\boldsymbol{\phi}},
\end{aligned}
$$

where the subscript $\mathrm{m}$ denotes meridional components, $\boldsymbol{\eta}_{\mathrm{m}}=\left(\eta_{s}, 0, \eta_{z}\right)$ and $\boldsymbol{\nabla} \times \overline{\boldsymbol{\eta}_{\mathrm{m}}}$ is in the azimuthal $\phi$-direction.

We emphasise that the results of this section apply to any vector $\boldsymbol{\eta}$ and not just to the ETHEL-displacement, which it defines elsewhere in this paper. 


\section{$5.1 \quad$ The model}

The essential idea behind the HEL-version of the nearly axisymmetric large- $R m$ dynamo was explained towards the end of section 1.4. Nevertheless, a few of the details need expanding upon in the light of the section 4.1 HEL-theory. In addition to the solenoidal consequences (135a-c) of the $\mathcal{J}=1$ assumption (134), our basic strategy is to adopt the glm-ETHELpostulates:

$$
\boldsymbol{u}=\overline{\boldsymbol{u}} \quad \text { and } \quad \overline{\boldsymbol{\eta}}=\mathbf{0}
$$

(see $(107 \mathrm{a}, \mathrm{d}))$ with the consequence that the fluctuating part of the real velocity $\boldsymbol{u}^{*}$ is accommodated by the fluctuating $\boldsymbol{\eta}$. The GLM-HEL-approach advocated by Andrews and McIntyre (1978a) builds on the assumption $\overline{\boldsymbol{\xi}}=\mathbf{0}$, which has attractions, but conflicts with our alternative glm-assumption $\overline{\boldsymbol{\eta}}=\mathbf{0}$. Indeed, when $\overline{\boldsymbol{\eta}}=\mathbf{0}$, it follows from (95a) that the mean HEL-displacement $\overline{\boldsymbol{\xi}}$ does not vanish but, correct to $\mathrm{O}\left(\eta^{2}\right)$, takes the value

$$
\bar{\xi} \approx \frac{1}{2} \overline{\boldsymbol{\eta} \cdot \boldsymbol{\nabla} \boldsymbol{\eta}} \quad \text { with } \quad \boldsymbol{\nabla} \cdot \boldsymbol{\eta}=0
$$

for our isochoric transformations (see (134)) implying $s^{-1} \partial_{\phi} \eta_{\phi}=-\boldsymbol{\nabla} \cdot \boldsymbol{\eta}_{\mathrm{m}}$. It therefore follows from (141) with $\boldsymbol{u}=\boldsymbol{\eta}$ that to the same order of accuracy the components are

$$
\overline{\xi_{s}} \approx \frac{1}{2}\left(\boldsymbol{\nabla} \cdot\left(\overline{\eta_{s} \boldsymbol{\eta}_{\mathrm{m}}}\right)-s^{-1} \overline{\eta_{\phi}^{2}}\right), \quad \overline{\xi_{\phi}} \approx \frac{1}{2} s^{-1} \boldsymbol{\nabla} \cdot\left(\overline{s \eta_{\phi} \boldsymbol{\eta}_{\mathrm{m}}}\right), \quad \overline{\xi_{z}} \approx \frac{1}{2} \boldsymbol{\nabla} \cdot\left(\overline{\eta_{z} \boldsymbol{\eta}_{\mathrm{m}}}\right) .(151 \mathrm{a}-\mathrm{c})
$$

To simplify matters, we assume henceforth that there is only one length scale $(\ell=) \mathcal{L}$ so that the small HEL-displacement $\boldsymbol{\xi}$, equivalently the ETHEL-displacement $\boldsymbol{\eta}$, is of magnitude

$$
\eta=\mathrm{O}(\varepsilon \mathcal{L}), \quad \varepsilon=R m^{-1 / 2}
$$

(see (50c), (49e)). In the absence of diffusion and when $q=\bar{q}$, the solutions of the perfect fluid equations (83a,d) may consistently contain no fluctuating part, i.e., $\boldsymbol{b}=\overline{\boldsymbol{b}}$ and $\theta=$ $\bar{\theta}$ respectively. Nevertheless with diffusion included, the terms $\boldsymbol{J}$ and $\kappa \boldsymbol{g} \cdot \boldsymbol{\nabla} \theta$ in $(127 \mathrm{~b}, \mathrm{c})$ necessarily contain fluctuating parts $\mathrm{O}\left(\mathfrak{K} \mathcal{L}^{-1} \varepsilon|\boldsymbol{b}|\right)$ and $\mathrm{O}\left(\kappa \mathcal{L}^{-1} \varepsilon|\theta|\right)$. In consequence small fluctuating parts of $\boldsymbol{b}^{\prime}$ and $\theta^{\prime}$ are generated:

$$
\boldsymbol{b}^{\prime} \equiv \boldsymbol{b}-\overline{\boldsymbol{b}}=\mathrm{O}\left(R m^{-1} \varepsilon|\boldsymbol{b}|\right), \quad \quad \theta^{\prime} \equiv \theta-\bar{\theta}=\mathrm{O}\left(P e^{-1} \varepsilon|\theta|\right) .
$$

These are generally sufficiently small that they may be neglected. Curiously, the main consequence of the discrepancy is in the evaluation of the Eulerian means $\overline{\boldsymbol{b}^{*}}$ and $\overline{\theta^{*}}$ which involve the small fluctuating HEL-parts $\boldsymbol{b}^{\prime}$ and $\theta^{\prime}$ (see $(110 \mathrm{a}, \mathrm{b})$ and $\left.(110 \mathrm{e}, \mathrm{f})\right)$. However, to maintain awareness of the fact that $\boldsymbol{b}^{\prime}$ and $\theta^{\prime}$ are neglected in the averaged forms of our governing equations, whenever appropriate we take advantage of the $\doteq$ notation (see (14)), which accommodates this neglect (as explained in the last paragraph of section 3 below (123b)). The remaining mean parts $\boldsymbol{u}(=\overline{\boldsymbol{u}}), \overline{\boldsymbol{b}}$ and $\bar{\theta}$ are axisymmetric with the mean velocity and magnetic field having toroidal-poloidal decompositions $\boldsymbol{u}=u_{\phi} \widehat{\boldsymbol{\phi}}+\boldsymbol{u}_{\mathrm{m}}$ and $\overline{\boldsymbol{b}}=\overline{b_{\phi}} \widehat{\boldsymbol{\phi}}+\overline{\boldsymbol{b}_{\mathrm{m}}}$, where

$$
\boldsymbol{u}_{\mathrm{m}}=s^{-1} \boldsymbol{\nabla}(s \psi) \times \widehat{\boldsymbol{\phi}}=\mathrm{O}\left(R m^{-1} u_{\phi}\right), \quad \overline{\boldsymbol{b}_{\mathrm{m}}}=s^{-1} \boldsymbol{\nabla}(s a) \times \widehat{\boldsymbol{\phi}}=\mathrm{O}\left(R m^{-1} \overline{b_{\phi}}\right)
$$

(cf. $(51 a, c))$.

We average the equation of motion (127a) and its curl, the $\phi$-components of which are

$$
\begin{gathered}
s^{-1} \mathrm{D}_{t}^{\mathrm{m}}\left(s\left(\overline{V_{\phi}}+\overline{\mathcal{R}_{\phi}}\right)\right)+2 \Omega u_{s} \doteq \rho_{0}^{-1} s^{-1} \overline{\boldsymbol{b}_{\mathrm{m}}} \cdot \nabla\left(s \overline{H_{\phi}}\right)+\overline{F_{\phi}}, \\
\mathrm{M}_{V}+\mathrm{M}_{\mathcal{R}}-\partial_{z}\left(2 \Omega u_{\phi}+s^{-1}\left(u_{\phi}^{2}-\left(\rho_{0} \mu\right)^{-1}{\overline{b_{\phi}}}^{2}\right)\right) \doteq \mathrm{L}+\left(\boldsymbol{\nabla} \times \overline{\boldsymbol{F}_{\mathrm{m}}}\right)_{\phi}
\end{gathered}
$$


respectively, where $\mathrm{D}_{t}^{\mathrm{m}} \equiv \partial_{t}+\boldsymbol{u}_{\mathrm{m}} \cdot \boldsymbol{\nabla}$ (cf. (66b)) and the inertial and Lorentz contributions

$$
\begin{aligned}
& \mathrm{M}_{V}=\left(s^{-1}\left(\boldsymbol{\nabla} \times \overline{\boldsymbol{V}_{\mathrm{m}}}\right)_{\phi}\right)+\left(\boldsymbol{\nabla}\left(s\left(\overline{V_{\phi}}-u_{\phi}\right)\right) \times \boldsymbol{\nabla}\left(s^{-1} u_{\phi}\right)\right)_{\phi}, \\
& \mathrm{M}_{\mathcal{R}}=s \mathrm{D}_{t}^{\mathrm{m}}\left(s^{-1}\left(\boldsymbol{\nabla} \times \overline{\mathcal{R}_{\mathrm{m}}}\right)_{\phi}\right)+\left(\boldsymbol{\nabla}\left(s \overline{\mathcal{R}_{\phi}}\right) \times \nabla\left(s^{-1} u_{\phi}\right)\right)_{\phi}, \\
& \rho_{0} \mathrm{~L} \doteq s \overline{\boldsymbol{b}_{\mathrm{m}}} \cdot \nabla\left(s^{-1}\left(\boldsymbol{\nabla} \times \overline{\boldsymbol{H}_{\mathrm{m}}}\right)_{\phi}\right)+\left(\nabla\left(s\left(\overline{H_{\phi}}-\mu^{-1} \overline{b_{\phi}}\right)\right) \times \nabla\left(s^{-1} \overline{b_{\phi}}\right)\right)_{\phi}
\end{aligned}
$$

to the vorticity equation (155b) are generally relatively small. In the case of $\mathbf{M}_{\mathcal{R}}$ and $\rho_{0} \mathrm{~L}$ we may estimate

$$
\begin{aligned}
\left(\nabla\left(s \overline{\mathcal{R}_{\phi}}\right) \times \nabla\left(s^{-1} u_{\phi}\right)\right)_{\phi} /\left(2 \Omega \partial_{z} u_{\phi}\right) & =\mathrm{O}\left(\varepsilon^{2}\right), \\
\left(\nabla\left(s\left(\mu \overline{H_{\phi}}-\overline{b_{\phi}}\right)\right) \times \nabla\left(s^{-1} \overline{b_{\phi}}\right)\right)_{\phi} /\left(\partial_{z}\left(s^{-1}{\overline{b_{\phi}}}^{2}\right)\right) & =\mathrm{O}\left(\varepsilon^{2}\right) .
\end{aligned}
$$

In the case of $\mathrm{M}_{V}$, a similar estimate to (156b) applies with $b_{\phi}$ and $\mu \overline{H_{\phi}}$ replaced by $u_{\phi}$ and $\overline{V_{\phi}}$. Nevertheless, that smallness is compounded by the geophysical possibility

$$
u_{\phi}^{2} \ll\left(\rho_{0} \mu\right)^{-1}{\overline{b_{\phi}}}^{2} .
$$

On a related matter, we may safely make the small Rossby number assumption $\mathcal{U} /(\mathcal{L} \Omega) \ll 1$ and ignore the term $s^{-1} u_{\phi}^{2}$ in (155b), which is then small compared with $2 \Omega u_{\phi}$. On the basis of all these approximations we obtain (178b) below.

In a similar way, we average the magnetic induction equation (127b) and its inverse curl, the $\phi$-components of which are

$$
\begin{aligned}
s^{-1} \mathrm{D}_{t}^{\mathrm{m}}(s a) & =\mathfrak{K}\left(-\mathrm{J}_{\phi}+\Delta_{1} a\right), \\
s \mathrm{D}_{t}^{\mathrm{m}}\left(s^{-1} \overline{b_{\phi}}\right) & =s \overline{\boldsymbol{b}_{\mathrm{m}}} \cdot \nabla\left(s^{-1} u_{\phi}\right)+\mathfrak{K}\left(-(\boldsymbol{\nabla} \times \mathbf{J})_{\phi}+\Delta_{1} \overline{b_{\phi}}\right),
\end{aligned}
$$

where $\Delta_{1}=\Delta-s^{-2}$ and

$$
\mathrm{J}_{\phi}=\mathfrak{K}^{-1} \overline{J_{\phi}}-\widehat{\boldsymbol{\phi}} \cdot\left(\boldsymbol{\nabla} \times \overline{\boldsymbol{b}_{\mathrm{m}}}\right), \quad(\boldsymbol{\nabla} \times \mathbf{J})_{\phi}=\widehat{\boldsymbol{\phi}} \cdot \boldsymbol{\nabla} \times\left(\mathfrak{K}^{-1} \overline{\boldsymbol{J}_{\mathrm{m}}}-\boldsymbol{\nabla} \times\left(\overline{b_{\phi}} \widehat{\boldsymbol{\phi}}\right)\right) .
$$

Likewise the average of the heat conduction equation $(127 \mathrm{c})$ is

$$
\mathrm{D}_{t}^{\mathrm{m}} \bar{\theta}=\kappa(\mathrm{H}+\Delta \bar{\theta})+\bar{q}
$$

where

$$
\mathrm{H} \doteq \nabla \cdot((\overline{\boldsymbol{g}}-\mathbf{I}) \cdot \nabla \bar{\theta})
$$

Now whereas we have

$$
(\boldsymbol{\nabla} \times \mathbf{J})_{\phi} /\left|\Delta_{1} \overline{b_{\phi}}\right|=\mathrm{O}\left(\varepsilon^{2}\right), \quad \mathrm{H} /|\Delta \bar{\theta}|=\mathrm{O}\left(\varepsilon^{2}\right),
$$

which are both small, it is significant that $\mathrm{J}_{\phi}$ involves $b_{\phi}$ so that

$$
\mathrm{J}_{\phi} /\left|\Delta_{1} a\right|=\mathrm{O}\left(R m \varepsilon^{2}\right)=\mathrm{O}(1)
$$

(see $(152 b))$. Then, unlike $-(\boldsymbol{\nabla} \times \mathbf{J})_{\phi}$ and $\mathbf{H}$ which are smaller than the primary diffusion terms $\Delta_{1} \overline{b_{\phi}}$ and $\Delta \bar{\theta}$, the source $-\mathrm{J}_{\phi}$ of Braginsky's $\alpha$-effect is comparable to $\Delta_{1} a$.

In $(171 \mathrm{a}, \mathrm{b})$ below we show that

$$
\begin{aligned}
-\mathrm{J}_{\phi} & \doteq s^{-1} \mathbf{p}_{\mathrm{m}} \cdot \boldsymbol{\nabla}(s a)+s^{-1} \aleph \overline{b_{\phi}}+\nabla^{p}\left(s^{-1}\left(\overline{g^{p q}}-\delta^{p q}\right) \nabla^{q}(s a)\right), \\
-(\boldsymbol{\nabla} \times \mathbf{J})_{\phi} & \doteq-s \boldsymbol{\nabla} \cdot\left(\mathbf{p}_{\mathrm{m}} s^{-1} \overline{b_{\phi}}\right)-\nabla^{p}\left(s^{-1} \mathrm{D}^{p q} \nabla^{q}(s a)\right)+\nabla^{p}\left(s^{-1}\left(\overline{g^{p q}}-\delta^{p q}\right) \nabla^{q}\left(s \overline{b_{\phi}}\right)\right), \\
\mathrm{H} & \doteq s^{-1} \nabla^{p}\left(s\left(\overline{g^{p q}}-\delta^{p q}\right) \nabla^{q} \bar{\theta}\right),
\end{aligned}
$$


in which

$$
s^{-1} \mathbf{p}_{\mathrm{m}} \cdot \boldsymbol{\nabla}(s a)=s^{-1} \mathrm{p}_{\mathrm{m}}^{q} \nabla^{q}(s a), \quad \quad s \boldsymbol{\nabla} \cdot\left(\mathbf{p}_{\mathrm{m}} s^{-1} \overline{b_{\phi}}\right)=\nabla^{q}\left(\mathrm{p}_{\mathrm{m}}^{q} \overline{b_{\phi}}\right)
$$

and where, in terms of the symmetric matrix $\gamma^{p q}$ (see (169b) below),

$$
s^{-1} \aleph=\overline{\gamma^{22}}, \quad \mathrm{p}_{\mathrm{m}}^{q}=\epsilon^{q 2 r} \overline{\gamma^{2 r}}, \quad \mathrm{D}^{p q}=\epsilon^{p 2 r} \epsilon^{q 2 s} \overline{\gamma^{r s}} .
$$

To summarise, $(158 \mathrm{a}, \mathrm{b})$ may be written compactly as

$$
\begin{aligned}
\partial_{t} a+s^{-1}\left(\boldsymbol{u}_{\mathrm{m}}-\mathfrak{K} \mathbf{p}_{\mathrm{m}}\right) \cdot \boldsymbol{\nabla}(s a) \doteq & \mathfrak{K}\left(s^{-1} \aleph \overline{b_{\phi}}+\nabla^{p}\left(s^{-1} \overline{g^{p q}} \nabla^{q}(s a)\right)\right), \\
\partial_{t} \overline{b_{\phi}}+s \boldsymbol{\nabla} \cdot\left(\left(\boldsymbol{u}_{\mathrm{m}}+\mathfrak{K} \mathbf{p}_{\mathrm{m}}\right) s^{-1} \overline{b_{\phi}}\right) \doteq & s \boldsymbol{b}_{\mathrm{m}} \cdot \boldsymbol{\nabla}\left(s^{-1} u_{\phi}\right) \\
& +\mathfrak{K} \nabla^{p}\left(s^{-1}\left(-\mathrm{D}^{p q} \nabla^{q}(s a)+\overline{g^{p q}} \nabla^{q}\left(s \overline{b_{\phi}}\right)\right)\right)
\end{aligned}
$$

(see Soward 1972, section 4, eqs. (4.4a,b), in which the term involving $\mathrm{D}^{p q}$ was omitted because of its very small size, as we explain in the discussion below $(174 \mathrm{a}, \mathrm{b}))$. Here $\mathbf{p}_{\mathrm{m}}$ has the interpretation of a pumping velocity $\mathfrak{K} \mathbf{p}_{\mathrm{m}}$ for $s^{-1} \overline{b_{\phi}}$ and a pumping velocity of the opposite sign $-\mathfrak{K} \mathbf{p}_{\mathrm{m}}$ for $s a$, while $s^{-1} \aleph$ and $\mathrm{D}^{p q}\left(=\mathrm{D}^{q p}\right)$ may be regarded as $\alpha$-effect coefficients that couple $\overline{b_{\phi}}$ to $a$. In fact, if $\left|s^{2} \nabla\left(s^{-1} u_{\phi}\right)\right|=\mathrm{o}\left(R m^{-1} u_{\phi}\right)$ and $\varepsilon=\mathrm{O}(1)$, there is the possibility of a $\alpha^{2}$-dynamo which relies on that coupling (see Soward 1972, section 3, particularly eqs. $(3.3 \mathrm{a}, \mathrm{b}))$.

Strictly our use of $\doteq$ in $(163 \mathrm{a}, \mathrm{b})$ is only correct after their multiplication by by $\mathfrak{K}^{-1}$, which brings their right-hand sides to the magnitudes of $J_{\phi}$ and $(\boldsymbol{\nabla} \times \mathbf{J})_{\phi}$ in $(161 \mathrm{a}, \mathrm{b})$. The spirit of its use, nevertheless, highlights the neglect of $\boldsymbol{b}^{\prime}$ and $\theta^{\prime}$ and draws awareness to possible parameter range limitations for the validity of our theory (see also the remarks following (175) below).

\section{$5.2 \quad$ The $\alpha$-effect}

The most important consequence of the Braginsky model is his $\alpha$-effect that stems from the term $J_{\phi}$ in the equation (158a) for the mean meridional magnetic field. To determine that, we need to first resolve $\mathfrak{K}^{-1} \boldsymbol{J}$ defined by (88) into its cylindrical polar coordinates

$$
\mathfrak{K}^{-1} J^{p}=\epsilon^{p q t} g^{q s}(\nabla \boldsymbol{b})^{s t}-\alpha^{p q} b^{q} .
$$

We substitute in the value of $(\boldsymbol{\nabla} \boldsymbol{b})^{\text {st }}$ determined by (145b) to obtain

$$
\begin{aligned}
\epsilon^{p q t} g^{q s}(\boldsymbol{\nabla} \boldsymbol{b})^{s t} & =\epsilon^{p q t} g^{q s}\left(\nabla^{s} b^{t}+s^{-1} \delta^{s 2} \epsilon^{3 u t} b^{u}\right) \\
& =\epsilon^{p q t} g^{q s} \nabla^{s} b^{t}+s^{-1}\left(\delta^{p 3} g^{q 2}-\delta^{p q} g^{32}\right) b^{q},
\end{aligned}
$$

where

$$
g^{q s}=\left(\boldsymbol{\nabla}^{L} \boldsymbol{x}\right)^{t q}\left(\boldsymbol{\nabla}^{L} \boldsymbol{x}\right)^{t s} .
$$

From the definition (89b) of the tensor $\boldsymbol{\alpha}$, its cylindrical polar coordinates are

$$
\begin{aligned}
\alpha^{p q} & =\epsilon^{p r s}\left(\boldsymbol{\nabla}^{L} \boldsymbol{x}\right)^{\operatorname{tr}}\left(\boldsymbol{\nabla}\left(\boldsymbol{\nabla}^{L} \boldsymbol{x}\right)\right)^{q t s} \\
& =\epsilon^{p r s}\left(\boldsymbol{\nabla}^{L} \boldsymbol{x}\right)^{\operatorname{tr}}\left(\nabla^{q}\left(\boldsymbol{\nabla}^{L} \boldsymbol{x}\right)^{t s}+s^{-1} \delta^{q 2}\left(\epsilon^{3 u t}\left(\boldsymbol{\nabla}^{L} \boldsymbol{x}\right)^{u s}+\epsilon^{3 u s}\left(\boldsymbol{\nabla}^{L} \boldsymbol{x}\right)^{t u}\right)\right),
\end{aligned}
$$

where we have used the result (146b). In view of the orthogonal nature of our transformations, standard Cartesian identities such as $\mathcal{J} \varepsilon_{i m n}\left(\boldsymbol{\nabla}^{L} \boldsymbol{x}\right)_{j m}\left(\boldsymbol{\nabla}^{L} \boldsymbol{x}\right)_{k n}=\varepsilon_{j k l}\left(\boldsymbol{\nabla} \boldsymbol{x}^{L}\right)_{i l}$ have their obvious cylindrical polar coordinate counterparts. In particular, when $\mathcal{J}=1$ this identity determines

$$
\epsilon^{p r s}\left(\nabla^{L} \boldsymbol{x}\right)^{t r}\left(\boldsymbol{\nabla}^{L} \boldsymbol{x}\right)^{u s}=\epsilon^{t u v}\left(\boldsymbol{\nabla} \boldsymbol{x}^{L}\right)^{p v} .
$$


So aided with the expressions (165b) and (166c), we may reduce (166b) to

$$
\alpha^{p q}=\epsilon^{p r s}\left(\boldsymbol{\nabla}^{L} \boldsymbol{x}\right)^{t r} \nabla^{q}\left(\boldsymbol{\nabla}^{L} \boldsymbol{x}\right)^{t s}-s^{-1} \delta^{q 2}\left(2 \mathcal{J}^{-1}\left(\boldsymbol{\nabla} \boldsymbol{x}^{L}\right)^{p 3}-\delta^{p 3} g^{t t}+g^{3 p}\right) .
$$

Together the results (165a) and (166d), the identity (164) becomes

$$
\mathfrak{K}^{-1} J^{p}=\epsilon^{p q t} g^{q s} \nabla^{s} b^{t}-s^{-1} \aleph^{p q} b^{q},
$$

where

$$
\begin{aligned}
s^{-1} \aleph^{p q}= & \alpha^{p q}-s^{-1}\left(\delta^{p 3} g^{q 2}-\delta^{p q} g^{32}\right) \\
= & \epsilon^{p r s}\left(\nabla^{L} \boldsymbol{x}\right)^{t r} \nabla^{q}\left(\nabla^{L} \boldsymbol{x}\right)^{t s} \\
& -s^{-1}\left(2 \delta^{q 2}\left(\nabla \boldsymbol{x}^{L}\right)^{p 3}-\delta^{q 2} \delta^{p 3} g^{t t}+\delta^{p 3} g^{q 2}+\delta^{q 2} g^{3 p}-\delta^{p q} g^{32}\right) \\
= & \epsilon^{p r s}\left(\boldsymbol{\nabla}^{L} \boldsymbol{x}\right)^{t r} \nabla^{q}\left(\nabla^{L} \boldsymbol{x}\right)^{t s}-s^{-1}\left(2 \delta^{q 2}\left(\boldsymbol{\nabla} \boldsymbol{x}^{L}\right)^{p 3}+\epsilon^{p r 2} \epsilon^{q s 3} g^{r s}\right) ;
\end{aligned}
$$

in going from the first to second equality we have used the well known identity $\epsilon^{p r u} \epsilon^{q s v}=$ $\delta^{p q}\left(\delta^{r s} \delta^{u v}-\delta^{r v} \delta^{u s}\right)+\delta^{p s}\left(\delta^{r v} \delta^{u q}-\delta^{r q} \delta^{u v}\right)+\delta^{p v}\left(\delta^{r q} \delta^{u s}-\delta^{r s} \delta^{u q}\right)$ with $u=2, v=3$. The result (167b) is the formula (2.30b) of Soward (1972), in which $\mu_{i j} \mapsto s^{-1} \aleph^{i j}, T_{p k}(\boldsymbol{X}) \mapsto\left(\boldsymbol{\nabla}^{L} \boldsymbol{x}\right)^{p k}$ and $a_{i j}(\boldsymbol{X}) \mapsto\left(\boldsymbol{\nabla} \boldsymbol{x}^{L}\right)^{j i}$ (note the interchange here of suffixes).

We can usefully cast the formula (90c), namely $\epsilon_{i j k} \alpha_{j k}=\nabla_{j} g_{i j}$, into its cylindrical polar coordinate form using (146b), which yields

$$
s^{-1} \nabla^{r}\left(s g^{p r}\right)+s^{-1} \epsilon^{3 s p} g^{s 2}=(\nabla \boldsymbol{g})^{r p r}=\epsilon^{p q r} \alpha^{q r}=s^{-1} \epsilon^{p q r} \aleph^{q r}+s^{-1} \epsilon^{3 s p} g^{s 2},
$$

from which we deduce

$$
\nabla^{r}\left(s g^{p r}\right)=\epsilon^{p q r} \aleph^{q r} \quad \text { equivalently } \quad \aleph^{p q}-\aleph^{q p}=\epsilon^{p q r} \nabla^{s}\left(s g^{r s}\right)
$$

(see Soward 1972, eq. (2.33)). Armed with the results $(168 \mathrm{~b}, \mathrm{c})$ we show in Appendix F that, under the assumption $b^{p}=\bar{b}^{p}$, the components of $\mathfrak{K}^{-1} \boldsymbol{J}$ may be expressed in the form

$$
\begin{aligned}
\mathfrak{K}^{-1} J^{p}= & -\nabla^{p}\left(\epsilon^{2 r q} g^{2 r} \overline{b^{q}}\right)+\epsilon^{p q 2} g^{q s} s^{-1} \nabla^{s}\left(s \overline{b^{2}}\right)+\delta^{p 2} \epsilon^{2 r q} \nabla^{s}\left(g^{r s} \overline{b^{q}}\right) \\
& -\left(\gamma^{p q}+\frac{1}{2} \epsilon^{p q r} s \nabla^{2}\left(s^{-1} g^{2 r}\right)\right) \overline{b^{q}}
\end{aligned}
$$

(see (F.5a)), where

$$
\gamma^{p q}=\frac{1}{2} \epsilon^{2 u t}\left(\epsilon^{r 2 q} \epsilon^{u p s}+\epsilon^{s 2 p} \epsilon^{u q r}\right) s \nabla^{t}\left(s^{-1} g^{r s}\right)+\frac{1}{2} s^{-1}\left(\aleph^{p q}+\aleph^{q p}\right)
$$

(see (F.6a,b) and Soward 1972, eq. (2.45)). We average $\boldsymbol{J}$ given by (169a), and substitute the toroidal-poloidal decomposition

$$
\overline{b^{p}}=\delta^{p 2} \overline{b_{\phi}}+s^{-1} \epsilon^{p r 2} \nabla^{r}(s a)
$$

(see (154b)) into both it and its curl:

$$
\begin{aligned}
\mathfrak{K}^{-1} \overline{J_{\phi}} & \doteq-\overline{\gamma^{2 r}} s^{-1} \epsilon^{r p 2} \nabla^{p}(s a)-\overline{\gamma^{22}} \overline{b_{\phi}}+\epsilon^{2 r q} \epsilon^{q t 2} \nabla^{s}\left(s^{-1} \overline{g^{r s}} \nabla^{t}(s a)\right), \\
\mathfrak{K}^{-1}(\boldsymbol{\nabla} \times \overline{\boldsymbol{J}})_{\phi} & \doteq \epsilon^{2 p r} \nabla^{p}\left(-\overline{\gamma^{r 2}} \overline{b_{\phi}}-\epsilon^{s t 2} s^{-1} \overline{\gamma^{r s}} \nabla^{t}(s a)+\epsilon^{r s 2} s^{-1} \overline{g^{s t}} \nabla^{t}\left(s \overline{b_{\phi}}\right)\right),
\end{aligned}
$$

which may be substituted into $(158 \mathrm{c}, \mathrm{d})$ to determine $\mathrm{J}_{\phi}$ and $(\boldsymbol{\nabla} \times \mathbf{J})_{\phi}$ defined by $(161 \mathrm{a}, \mathrm{b})$. The upshot of these substitutions into $(158 \mathrm{a}, \mathrm{b})$ is the $\phi$-averaged dynamo equations $(163 \mathrm{a}, \mathrm{b})$.

We now derive the leading order contributions to $\mathfrak{K}^{-1} \overline{J_{\phi}}$ and $\mathfrak{K}^{-1}(\boldsymbol{\nabla} \times \overline{\boldsymbol{J}})_{\phi}$ defined by (171a,b) for small amplitude displacements, $\boldsymbol{\eta}=\mathrm{O}(\varepsilon \mathcal{L})$ (see $(152 \mathrm{a}, \mathrm{b})$ ), with $\varepsilon \ll 1$. To begin we note that the $\phi$ averages of the coefficients involving only the fluctuations are small. They are

$$
\overline{\gamma^{p q}}=\mathrm{O}\left(\mathcal{L}^{-1} \varepsilon^{2}\right), \quad \overline{g^{p q}}-\delta^{p q}=\mathrm{O}\left(\varepsilon^{2}\right)
$$


implying

$$
\mathbf{p}_{\mathrm{m}}=\mathrm{O}\left(\mathcal{L}^{-1} \varepsilon^{2}\right), \quad \aleph=s \overline{\gamma^{22}}=\mathrm{O}\left(\varepsilon^{2}\right), \quad \mathrm{D}^{p q}=\mathrm{O}\left(\mathcal{L}^{-1} \varepsilon^{2}\right) .
$$

The main contributions made by the final terms of $(171 \mathrm{a}, \mathrm{b})$ are obtained from the largest parts, $\delta^{r s}$ and $\delta^{s t}$, of $g^{r s}$ and $g^{s t}$ respectively, and are therefore

$$
\epsilon^{2 s q} \epsilon^{q t 2} \nabla^{s}\left(s^{-1} \nabla^{t}(s a)\right)=-\Delta_{1} a, \quad \quad \epsilon^{2 p r} \nabla^{p}\left(\epsilon^{r s 2} s^{-1} \overline{g^{s t}} \nabla^{t}\left(s \overline{b_{\phi}}\right)\right)=-\Delta_{1} \overline{b_{\phi}},
$$

where $\Delta_{1} \bullet=\left(\nabla^{2}-s^{-2}\right) \bullet=\nabla^{r}\left(s^{-1} \nabla^{r}(s \bullet)\right.$ ) (see (52c)). This explains how the last terms on the right-hand sides of $(158 \mathrm{a}, \mathrm{b})$ and $(161 \mathrm{a}, \mathrm{b})$ arise. In $(161 \mathrm{a}, \mathrm{b})$ the terms involving $\overline{g^{p q}}-\delta^{p q}$ and $\mathbf{p}_{\mathrm{m}}$ are $\mathrm{O}\left(R m^{-1}\right)$, while the term involving $\mathrm{D}^{p q}$ in $(161 \mathrm{~b})$ is even smaller, $\mathrm{O}\left(R m^{-2}\right)$. According to (154b), we have

$$
|\nabla a| /\left|\overline{b_{\phi}}\right|=\mathrm{O}\left(R m^{-1}\right)
$$

so that, bearing in mind (160c), the term $\overline{\gamma^{22}} \overline{b_{\phi}}$ in (171a) must be retained, even though the other terms in $(171 \mathrm{a}, \mathrm{b})$ involving $\overline{\gamma^{r s}} \overline{b_{\phi}}$ are negligible. Those small effects were identified by Soward (1972). Nevertheless, other small effects originating from $\boldsymbol{b}^{\prime}$ may lead to comparably sized contributions to the $\alpha$-tensor (see Soward 1972, section 4). Higher order effects have also been investigated by Cupal (1984).

On retaining only the leading order terms, $(163 \mathrm{a}, \mathrm{b})$ reduce to

$$
\begin{aligned}
\mathrm{D}_{t}^{\mathrm{m}}(s a) & \doteqdot \mathfrak{K}\left(\aleph b_{\phi}+s \Delta_{1} a\right), \\
\mathrm{D}_{t}^{\mathrm{m}}\left(s^{-1} b_{\phi}\right) & \doteqdot \boldsymbol{b}_{\mathrm{m}} \cdot \nabla\left(s^{-1} u_{\phi}\right)+\mathfrak{K} s^{-1} \Delta_{1} b_{\phi},
\end{aligned}
$$

where from (167c), (169b) and (173b)

$$
s \overline{\gamma^{22}}=\aleph \equiv \overline{\aleph^{22}}=\epsilon^{2 r s} \overline{\left(\nabla^{L} \boldsymbol{x}\right)^{t r} \partial_{\phi}\left(\nabla^{L} \boldsymbol{x}\right)^{t s}} .
$$

The physically more revealing form

$$
\aleph=-\frac{1}{2 \pi} \oint_{\mathcal{C}^{*}}\left(\nabla^{L} \times \frac{1}{s} \frac{\partial \boldsymbol{x}^{L}}{\partial \phi}\right) \cdot \mathrm{d} \boldsymbol{x}^{L}
$$

is established in (186a,b) below. Use of the expansion (96c) and the formula (145b) leads to the approximate result

$$
\aleph \approx \epsilon^{2 r s} \overline{(\boldsymbol{\nabla} \boldsymbol{\eta})^{t r} \partial_{\phi}(\boldsymbol{\nabla} \boldsymbol{\eta})^{t s}}=2\left(\overline{\left(\nabla^{t} \eta^{3}\right) \partial_{\phi}\left(\nabla^{t} \eta^{1}\right)}-\overline{\left(\nabla^{2} \eta^{2}\right)\left(\nabla^{2} \eta^{3}\right)}\right)
$$

(see Soward 1972, eq. (C11) $R \Gamma_{22} \mapsto s^{-1} \aleph$ ). Since $\boldsymbol{\nabla} \cdot \boldsymbol{\eta}=0$, we may express the result in the form

$$
\aleph \approx 2\left(\overline{\frac{\partial \eta_{z}}{\partial s} \frac{\partial^{2} \eta_{s}}{\partial \phi \partial s}}+\overline{\frac{1}{s^{2}} \frac{\partial \eta_{z}}{\partial \phi} \frac{\partial^{2} \eta_{s}}{\partial \phi^{2}}}+\overline{\frac{\partial \eta_{z}}{\partial z} \frac{\partial^{2} \eta_{s}}{\partial \phi \partial z}}\right)+\frac{2}{s} \overline{\left(\frac{1}{s} \frac{\partial\left(s \eta_{s}\right)}{\partial s}+\frac{\partial \eta_{z}}{\partial z}\right) \frac{\partial \eta_{z}}{\partial \phi}},
$$

which corrects the erroneous expression (C12) of Soward (1972) $\left(\boldsymbol{\xi}_{\mathrm{m}} \simeq \boldsymbol{\eta}_{\mathrm{m}}\right)$, but agrees with Braginsky's original form (54a) for $\aleph^{\ddagger}$, in the sense that $\boldsymbol{\zeta}_{\mathrm{m}} \simeq \boldsymbol{\eta}_{\mathrm{m}}$ and $\aleph^{\ddagger} \approx \aleph$.

\subsection{The momentum equation}

Generally because of the dominance of the Coriolis force in the momentum equation, we may approximate $(155 \mathrm{a}, \mathrm{b})$ by the simple forms

$$
\begin{gathered}
2 \Omega u_{s} \doteqdot\left(\rho_{0} \mu\right)^{-1} s^{-1} \overline{\boldsymbol{b}_{\mathrm{m}}} \cdot \boldsymbol{\nabla}\left(s \overline{b_{\phi}}\right)+\overline{F_{\phi}} \\
-\partial_{z}\left(2 \Omega u_{\phi}-\left(\rho_{0} \mu\right)^{-1} s^{-1}{\overline{b_{\phi}}}^{2}\right) \doteqdot\left(\boldsymbol{\nabla} \times \overline{\boldsymbol{F}_{\mathrm{m}}}\right)_{\phi}
\end{gathered}
$$


as explained in section 5.1. We have made the approximation $\mu \overline{H_{\phi}} \doteqdot \overline{b_{\phi}}$ in $\phi$-component of the averaged HEL momentum equation (178a), which is essentially the ETHEL-form derived by Tough and Roberts (see Tough and Roberts 1968, eq. (47)). Nevertheless a complication arises upon integrating (178a) with respect to $z$ between one boundary and the other. The issue was raised by Taylor (1963) and its immediate consequence is referred to as Taylor's condition. Essentially, the constraint of mass continuity eliminates the dominant Coriolis term $2 \Omega u_{s}$. So smaller terms particularly involving $\overline{V_{\phi}}, \overline{\mathcal{R}_{\phi}}$ and $\mu \overline{H_{\phi}}$ appearing in (155a), which are neglected in (178a), may become significant. For that reason, we now consider their ETHEL-expansions.

Consideration of $\boldsymbol{V}=\left(\boldsymbol{\nabla} \boldsymbol{x}^{L}\right) \cdot\left(\mathrm{D}_{t} \boldsymbol{x}^{L}\right)$ (see $(66 \mathrm{a}, \mathrm{b})$ and (67a) noting $\boldsymbol{V}^{*}=\boldsymbol{u}^{*}$ ) leads to

$$
\boldsymbol{V}-\boldsymbol{u}=\partial_{t} \boldsymbol{\xi}+(\boldsymbol{\nabla} \boldsymbol{\xi}) \cdot\left(\partial_{t} \boldsymbol{\xi}\right)+\boldsymbol{u} \cdot \boldsymbol{\nabla} \boldsymbol{\xi}+(\boldsymbol{\nabla} \boldsymbol{\xi}) \cdot \boldsymbol{u}+(\boldsymbol{\nabla} \boldsymbol{\xi}) \cdot(\boldsymbol{u} \cdot \boldsymbol{\nabla} \boldsymbol{\xi}) .
$$

We approximate $\boldsymbol{u} \simeq u_{\phi} \widehat{\boldsymbol{\phi}}$ on the basis that $\boldsymbol{u}_{\mathrm{m}}=\mathrm{O}\left(\varepsilon^{2} u_{\phi}\right)$ and use (47a) to obtain

$$
V_{\phi}-u_{\phi} \approx \partial_{t} \xi_{\phi}+s^{-1}\left(\partial_{\phi} \boldsymbol{\xi}+\widehat{\boldsymbol{z}} \times \boldsymbol{\xi}\right) \cdot\left(\partial_{t} \boldsymbol{\xi}\right)+2 s^{-1}\left(\partial_{\phi} \xi_{\phi}+\xi_{s}\right) u_{\phi}+s^{-2}\left|\partial_{\phi} \boldsymbol{\xi}+\widehat{\boldsymbol{z}} \times \boldsymbol{\xi}\right|^{2} u_{\phi},
$$

in which $\boldsymbol{\xi} \approx \boldsymbol{\eta}+\frac{1}{2} \boldsymbol{\eta} \cdot \boldsymbol{\nabla} \boldsymbol{\eta}$ (see (95a)). Whence, correct to $\mathrm{O}\left(\eta^{2}\right)$, the average of (180) is

$$
\overline{V_{\phi}}-u_{\phi} \approx \partial_{t} \overline{\xi_{\phi}}+s^{-1} \overline{\left(\partial_{\phi} \boldsymbol{\eta}+\widehat{\boldsymbol{z}} \times \boldsymbol{\eta}\right) \cdot\left(\partial_{t} \boldsymbol{\eta}\right)}+s^{-1}\left(2 \overline{\xi_{s}}+s^{-1} \overline{\left|\partial_{\phi} \boldsymbol{\eta}+\widehat{\boldsymbol{z}} \times \boldsymbol{\eta}\right|^{2}}\right) u_{\phi},
$$

where $\overline{\xi_{s}}$ and $\overline{\xi_{\phi}}$ are given by (151a,b) respectively. The average of the $\phi$-component of (136) determines

$$
\overline{\mathcal{R}_{\phi}} \approx \Omega \overline{\Xi_{z}}, \quad \text { where } \quad \Xi_{z} \equiv \widehat{\phi} \cdot\left(\boldsymbol{\eta}_{\mathrm{m}} \times \partial_{z} \boldsymbol{\eta}_{\mathrm{m}}\right) .
$$

The average of the $\phi$-component of $\mu \boldsymbol{H}=\left(\boldsymbol{\nabla} \boldsymbol{x}^{L}\right) \cdot\left(\boldsymbol{b} \cdot \boldsymbol{\nabla} \boldsymbol{x}^{L}\right)$ (see (61b) and (67b) noting $\mu \boldsymbol{H}^{*}=\boldsymbol{b}^{*}$ and $\mathcal{J}=1$ ) leads by steps, analogous to (179) and (180), to

$$
\mu \overline{H_{\phi}}-\overline{b_{\phi}} \approx s^{-1}\left(2 \overline{\xi_{s}}+s^{-1} \overline{\left|\partial_{\phi} \boldsymbol{\eta}+\widehat{\boldsymbol{z}} \times \boldsymbol{\eta}\right|^{2}}\right) b_{\phi},
$$

similar to (181).

It is important to realise that

$$
\overline{V_{\phi}}=\frac{1}{2 \pi s} \oint_{\mathcal{C}^{*}} \boldsymbol{u}^{* L} \cdot \mathrm{d} \boldsymbol{x}^{L}, \quad \mu \overline{H_{\phi}}=\frac{1}{2 \pi s} \oint_{\mathcal{C}^{*}} \boldsymbol{b}^{* L} \cdot \mathrm{d} \boldsymbol{x}^{L},
$$

where $\mathcal{C}^{*}$ is the loop composed of the points $\boldsymbol{x}^{L}(\boldsymbol{x}, t)$ generated by $\boldsymbol{x}=(s, \phi, z)$ for $0 \leq \phi<2 \pi$ at fixed $(s, z)$. Likewise we have

$$
\mathfrak{K}^{-1} \overline{J_{\phi}}=\frac{1}{2 \pi s} \oint_{\mathcal{C}^{*}}\left(\nabla^{L} \times \boldsymbol{b}^{* L}\right) \cdot \mathrm{d} \boldsymbol{x}^{L} .
$$

For the particular choice of an azimuthal HEL magnetic field $b^{q}=\delta^{q 2} \overline{b_{\phi}}$, we have on noting (118) and (158c) that the average of (167a) with $p=2$ (recall $\left.\overline{\aleph^{22}}=\aleph\right)$ gives

$$
-\mathfrak{K}^{-1} \mathcal{E}_{\phi}=J_{\phi}=\mathfrak{K}^{-1} \overline{J_{\phi}}=-s^{-1} \aleph \overline{b_{\phi}} \widehat{\phi} \quad\left(\boldsymbol{b}=\overline{b_{\phi}} \widehat{\phi}\right) .
$$

Substitution of (186a) into (185) leads to

$$
-s^{-1} \aleph \overline{b_{\phi}}=\frac{1}{2 \pi s} \oint_{\mathcal{C}^{*}}\left(\nabla^{L} \times \frac{\overline{b_{\phi}}}{s} \frac{\partial \boldsymbol{x}^{L}}{\partial \phi}\right) \cdot \mathrm{d} \boldsymbol{x}^{L} \quad\left(\boldsymbol{b}^{* L}=\frac{\overline{b_{\phi}}}{s} \frac{\partial \boldsymbol{x}^{L}}{\partial \phi}\right),
$$

from which (177b) follows immediately. We also note that

$$
\overline{G_{\phi}}=\frac{1}{2 \pi s} \oint_{\mathcal{C}^{*}} \boldsymbol{G}^{* L} \cdot \mathrm{d} \boldsymbol{x}^{L}=0,
$$

since the gravitational acceleration $\boldsymbol{G}^{*}$ is the gradient of a potential:

$$
\boldsymbol{G}^{*}=-\nabla \chi^{*} \quad \Longrightarrow \quad \boldsymbol{G}=-\nabla \chi, \quad \text { where } \quad \chi=\chi^{* L} .
$$


In the absence of thermal diffusion this implies that

$$
\overline{F_{\phi}}=\frac{1}{2 \pi s} \oint_{\mathcal{C}^{*}} \boldsymbol{F}^{* L} \cdot \mathrm{d} \boldsymbol{x}^{L}=0,
$$

when

$$
\boldsymbol{F}^{*}=-\theta^{*} \boldsymbol{G}^{*} \quad \text { and } \quad \theta^{* L} \equiv \theta=\bar{\theta} .
$$

However, with small but finite thermal diffusion $P e \gg 1$, a non-zero contribution $\overline{F_{\phi}}$ originates from the small fluctuating part of $\theta$, which is given by

$$
\overline{F_{\phi}}=s^{-1} \overline{\theta^{\prime} \partial_{\phi} \chi}, \quad \quad \text { where } \quad \theta^{\prime} \ll \bar{\theta} .
$$

This means that the non-zero value of $\overline{F_{\phi}}$ is a high order effect that only occurs after temperature has diffused slightly.

Provided that $\left|u_{\phi}\right| \ll\left(\rho_{0} \mu\right)^{-1 / 2}\left|\overline{b_{\phi}}\right|$ (see (157)), which may approximate conditions in the Earth's core (but see Roberts and Aurnou 2012, for more discussion), we neglect the contribution $s^{-1} \boldsymbol{u}_{\mathrm{m}} \cdot \boldsymbol{\nabla}\left(s \overline{V_{\phi}}\right)$ to the term $s^{-1} \mathrm{D}_{t}^{\mathrm{m}}\left(s \overline{V_{\phi}}\right)$ in (155a), but retain $\partial_{t} \overline{V_{\phi}}$ in case the time scale is short, so leaving

$$
\partial_{t}\left(\overline{V_{\phi}}+\overline{\mathcal{R}_{\phi}}\right)+s^{-1} \boldsymbol{u}_{\mathrm{m}} \cdot \boldsymbol{\nabla}\left(s \overline{\mathcal{R}_{\phi}}\right)+2 \Omega u_{s} \doteqdot \rho_{0}^{-1} s^{-1} \overline{\boldsymbol{b}_{\mathrm{m}}} \cdot \boldsymbol{\nabla}\left(s \overline{H_{\phi}}\right)+\overline{F_{\phi}} .
$$

Here

$$
\left|s^{-1} \boldsymbol{u}_{\mathrm{m}} \cdot \boldsymbol{\nabla}\left(s \overline{\mathcal{R}_{\phi}}\right)\right| /\left|2 \Omega u_{s}\right|=\mathrm{O}\left(\varepsilon^{2}\right)
$$

suggesting that $s^{-1} \boldsymbol{u}_{\mathrm{m}} \cdot \boldsymbol{\nabla}\left(s \overline{\mathcal{R}_{\phi}}\right)$ is negligible too. On that basis also ignoring for the moment the time derivatives, (191) reduces to (178a).

If we denote the location of the top and bottom boundaries of the axisymmetric container $\mathcal{S}$ (assumed spherical) by $z=h_{ \pm}(s)$, we note that continuity of mass flux $\boldsymbol{\nabla} \cdot \boldsymbol{u}=0$ (see (135a)) implies that $\int_{h_{-}}^{h_{+}} u_{s} \mathrm{~d} z=0$ provided that any boundary layer mass fluxes, such as carried by Ekman layers, are ignored so that $\boldsymbol{u} \cdot \mathrm{d} \boldsymbol{S}=0$ on $\mathcal{S}$ (see (137a)). Consequently following the $z$-integration of (191), the contribution from the large term $2 \Omega u_{s}$ vanishes. So under that $z$-integration, we retain the apparently small numerator of (192), as well as the small time derivative terms, to obtain

$$
\begin{aligned}
\frac{\partial}{\partial t} \int_{h_{-}}^{h_{+}}\left(\overline{V_{\phi}}+\overline{\mathcal{R}_{\phi}}\right) \mathrm{d} z+\frac{1}{s^{2}} \frac{\partial}{\partial s} \int_{h_{-}}^{h_{+}} s^{2} u_{\phi} \overline{\mathcal{R}_{\phi}} \mathrm{d} z \\
\quad \doteqdot \rho_{0}^{-1}\left(\frac{1}{s^{2}} \frac{\partial}{\partial s} \int_{h_{-}}^{h_{+}} s^{2} \overline{b_{\phi}} \overline{H_{\phi}} \mathrm{d} z+\frac{1}{2 \pi s} \frac{\mathrm{d} S}{\mathrm{~d} s}\left(\left[\overline{H_{\phi}} \overline{\boldsymbol{b}_{\mathrm{m}}} \cdot \boldsymbol{n}\right]_{h_{+}}+\left[\overline{H_{\phi}} \overline{\boldsymbol{b}_{\mathrm{m}}} \cdot \boldsymbol{n}\right]_{h_{-}}\right)\right) \\
\quad+\int_{h_{-}}^{h_{+}} \overline{F_{\phi}} \mathrm{d} z
\end{aligned}
$$

where $S$ is the surface area of the spherical cap out as far as the radius $s$ and $\boldsymbol{n}$ is the outward unit normal. In (193), $\overline{V_{\phi}}$ and $\mu \overline{H_{\phi}}$ may be reasonably be approximated by $u_{\phi}$ and $b_{\phi}$ respectively, as in (178a). The equation can be used to discuss torsional oscillations in the presence of underlying non-axisymetric waves, whose influence is encompassed by the term involving $\overline{\mathcal{R}_{\phi}} \approx \Omega \widehat{\boldsymbol{\phi}} \cdot \overline{\left(\boldsymbol{\eta} \times \partial_{z} \boldsymbol{\eta}\right)}$ (see $(182 \mathrm{a}, \mathrm{b})$ ).

The dominant force balance in the $\phi$-averaged azimuthal HEL momentum and vorticity equations are (178a,b), which are supplemented by the generalised Taylor's condition (193). Now whereas Tough and Roberts (1968) derived, using Braginsky's original method, the effective-variable form (178a) (their eq. (47)), they made the assumption $\overline{\boldsymbol{F}^{*}}=\mathbf{0}$ (their eq. (26)). To leading order the consequence of this assumption is that $\left(\boldsymbol{\nabla} \times \overline{\boldsymbol{F}_{\mathrm{m}}}\right)_{\phi} \simeq 0$ 
too leading to their eq. (31). In our case with the buoyancy force $(189 \mathrm{~b}, \mathrm{c})$ included (178b) becomes

$$
-\partial_{z}\left(2 \Omega u_{\phi}-\left(\rho_{0} \mu\right)^{-1} s^{-1}{\overline{b_{\phi}}}^{2}\right) \doteqdot\left(\boldsymbol{\nabla} \times \overline{\boldsymbol{F}_{\mathrm{m}}}\right)_{\phi} \doteq(\overline{\nabla \theta \times \nabla \chi})_{\phi} \approx(\boldsymbol{\nabla} \bar{\theta} \times \boldsymbol{\nabla} \bar{\chi})_{\phi}
$$

on the basis that $\theta^{\prime}=\mathrm{O}\left(P e^{-1} \varepsilon|\bar{\theta}|\right)$ (see (153b)) and $\chi^{\prime}=\mathrm{O}(\varepsilon|\bar{\chi}|)$. Here the right-hand side is simply the usual thermal wind contribution $-\left(\nabla \overline{\theta^{*}} \times G^{*}\right)_{\phi}$. With $\overline{F_{\phi}}$ negligible in the mean azimuthal HEL momentum equation (178a), the thermal wind source in the mean azimuthal HEL vorticity (194) is the only remaining mechanical energy source, while the $\alpha$-effect $\aleph \overline{b_{\phi}}$ and $\omega$-effect $\overline{\boldsymbol{b}_{\mathrm{m}}} \cdot \boldsymbol{\nabla}\left(s^{-1} u_{\phi}\right)$ terms in the magnetic induction equations (176a,b) provide the corresponding electrical energy sources. This is all quite standard and corresponds to the traditional mean-field approach. The only new feature is the origin of the $\alpha$-effect encapsulated by the mean-field coefficient $\aleph$.

\section{Conclusions}

We should emphasise a crucial difference in the implementations of traditional HEL and ETHEL to mean-field theory. Though both assume that $\boldsymbol{u}=\overline{\boldsymbol{u}}$, in the former, Andrews and McIntyre (1978a) make the GLM-postulate

$$
\overline{\boldsymbol{\xi}}=\mathbf{0} \quad \Longrightarrow \quad \overline{\boldsymbol{u}^{* L}}=\boldsymbol{u} \quad(\overline{\boldsymbol{\eta}} \neq \mathbf{0}),
$$

whereas, in the latter, we advocate instead the glm-postulate

$$
\overline{\boldsymbol{\eta}}=\mathbf{0} \quad \Longrightarrow \quad \overline{\boldsymbol{u}^{* L}}=\boldsymbol{u}+\mathrm{D}_{t} \overline{\boldsymbol{\xi}} \quad(\overline{\boldsymbol{\xi}} \neq \mathbf{0})
$$

to fulfil the Eulerian objective of Holm (2002) (see section 1.5). Both the HEL and ETHEL theories build on the Lagrangian position $\boldsymbol{x}^{L}=\boldsymbol{x}^{L}(\boldsymbol{x}, t)$. The value of $\boldsymbol{x}^{L}$ is constrained, by whichever of the postulates $\overline{\boldsymbol{\xi}}=\mathbf{0}(195 \mathrm{a})$ and $\overline{\boldsymbol{\eta}}=\mathbf{0}$ (195c) is made, and will certainly take on different values in each of the two cases. This means that the averages of $\overline{\boldsymbol{u}^{* L}}$, taken at different locations $\boldsymbol{x}^{L}=\boldsymbol{x}+\boldsymbol{\xi}$, must differ also. These differences spill over into the values of the other HEL-variables particularly $\boldsymbol{u}$, which must all necessarily differ slightly at $\mathrm{O}\left(\xi^{2}\right)$. To make contact with the GLM-theory of Andrews and McIntyre (1978a), we note that

$$
\boldsymbol{V}-\boldsymbol{u}^{* L}=(\boldsymbol{\nabla} \boldsymbol{\xi}) \cdot \boldsymbol{u}^{* L}, \quad \boldsymbol{\mathcal { R }}-2 \boldsymbol{\Omega} \times \boldsymbol{\xi}=(\boldsymbol{\nabla} \boldsymbol{\xi}) \cdot(\boldsymbol{\Omega} \times \boldsymbol{\xi}),
$$

since $\boldsymbol{V}=\left(\boldsymbol{\nabla} \boldsymbol{x}^{L}\right) \cdot \boldsymbol{V}^{* L}=\left(\boldsymbol{\nabla} \boldsymbol{x}^{L}\right) \cdot \boldsymbol{u}^{* L}$ and see the near right-hand side of (131). On making the GLM-postulate (195a) $\overline{\boldsymbol{\xi}}=\mathbf{0}$, which implies that both $\overline{\boldsymbol{u}^{* L}}=\boldsymbol{u}$ and $\overline{\boldsymbol{\mathcal { R }}}=\overline{(\boldsymbol{\nabla} \boldsymbol{\xi}) \cdot(\boldsymbol{\Omega} \times \boldsymbol{\xi})}$, we recover their pseudomomentum interpretation $\mathbf{p}=\mathbf{p}^{0}+\mathbf{p}^{\Omega}$, where $\mathbf{p}^{0} \equiv-\overline{(\boldsymbol{\nabla} \boldsymbol{\xi}) \cdot \boldsymbol{u}^{* L}}=$ $\boldsymbol{u}-\overline{\boldsymbol{V}}$ and $\mathbf{p}^{\Omega} \equiv-\overline{(\boldsymbol{\nabla} \boldsymbol{\xi}) \cdot(\boldsymbol{\Omega} \times \boldsymbol{\xi})}=-\overline{\mathcal{R}}$ as defined by (B.3) and (B.6) respectively in Appendix B of Andrews and McIntyre (1978a). When instead the glm-postulate (195c) $\overline{\boldsymbol{\eta}}=\mathbf{0}$ is made implying $\overline{\boldsymbol{\xi}} \neq \mathbf{0}$, the associated pseudomomentum vectors $\boldsymbol{u}-\overline{\boldsymbol{V}}=-\mathrm{D}_{t} \overline{\boldsymbol{\xi}}-\overline{(\boldsymbol{\nabla} \boldsymbol{\xi}) \cdot \boldsymbol{u}^{* L}}$ and $-\overline{\mathcal{R}}=-2 \boldsymbol{\Omega} \times \overline{\boldsymbol{\xi}}-\overline{(\boldsymbol{\nabla} \boldsymbol{\xi}) \cdot(\boldsymbol{\Omega} \times \boldsymbol{\xi})}$ identified by the averaged HEL momentum equation (127a) certainly differ from the pseudomomentum vectors $\mathbf{p}^{0}$ and $\mathbf{p}^{\Omega}$ of Andrews and McIntyre (1978a). All these differences are not in conflict but rather reflect different interpretations of the physical system (further insights are provided in sections 4.1, 4.2 and 5 of Soward and Roberts 2010, which we refrain from repeating here).

The issue that must be addressed is how to solve the HEL-equations governing the meanfields, such as the Braginsky dynamo equations (176a,b) and the corresponding momentum equations $(178 \mathrm{a}, \mathrm{b})$, even in their approximate forms, to determine the actual solution of the physical system. Obviously, knowledge of the HEL-displacement $\boldsymbol{\xi}$ (equivalently the ETHELdisplacement $\boldsymbol{\eta}$ ) is needed. Then it is possible to determine the ETHEL-forms of the HELvariables using the Lie derivative Taylor expansion (98a) of the Eulerian forms and vice versa 
using the inverse expansion (98b) (also (105a-f)), where the Lie derivatives are summarised by (99a-e).

As we explained in section 1.4, Braginsky (1964a,b) achieved a form of ETHEL-equations for his proposed kinematic dynamo from an Eulerian point of view. It is only through our intermediate HEL-construction that the physical content of his effective-variables (51a-f) involving $\varpi^{\ddagger}$ (see $\left.(51 \mathrm{~g})\right)$ and the $\alpha$-effect $\mathfrak{K} s^{-1} \aleph^{\ddagger}$ in $(52 \mathrm{~d})$ becomes transparent. The relation of his approach, which naturally builds on an assumed form of the effective-displacement vector field $\boldsymbol{\zeta}$ introduced in section 1.1, to the ETHEL-description that relies on $\boldsymbol{\eta}$ is considered in Appendix G. There it is concluded that differences are largely negligible at the order needed to close the equations at leading order. From that point of view, on introduction of the ETHEL-displacement $\boldsymbol{\eta}$ into Braginsky's mean-field dynamo equations $(52 \mathrm{a}, \mathrm{b})$ with $\alpha$-coefficient $s^{-1} \aleph^{\ddagger}$ (see (54a)), they become the glm dynamo equations (176a,b) with $\alpha$ coefficient $s^{-1} \aleph$ (see $\left.(177 \mathrm{c}, \mathrm{d})\right)$. Consideration of the HEL mean momentum equations $(178 \mathrm{a}, \mathrm{b})$ raises similar issues to those just described for the mean magnetic induction equation.

The key matter to be resolved is the determination of the displacement fields $\boldsymbol{\xi}, \boldsymbol{\eta}$ or $\boldsymbol{\zeta}$. As often done in traditional mean-field theories, we may assume that the parameters that relate the Eulerian to the HEL or ETHEL means such as $\aleph$ and $\varpi$ (see (177d) and (G.14a) respectively) are given, i.e. traditionaly one would claim that the micro-dynamics was prescribed and one would say that one was simply solving the macro-dynamics. A more ambitious strategy would be to solve the equations for the fluctuating quantities together with those of the mean-fields simultaneously. The point of ETHEL is to provide a formulation of the HEL governing equations in Eulerian form as in Braginsky's original derivation. Thus the equations governing the fluctuations are at lowest order those normally encountered in conventional Eulerian studies. Then possibly with even the diffusivities ignored, the fluctuating velocity $\boldsymbol{u}^{* \prime}$ may be converted into the appropriate value of one of the displacement fields $\boldsymbol{\xi}, \boldsymbol{\eta}$ or $\boldsymbol{\zeta}$. This is an ambitious project yet to be undertaken.

A surprising feature of the glm-approach is first made clear by the formula (40a,c):

$$
\overline{\boldsymbol{b}^{\dagger}} \approx \overline{\boldsymbol{b}^{*}}-\frac{1}{2} \boldsymbol{\nabla} \times\left(\overline{\boldsymbol{\zeta} \times\left(\boldsymbol{b}^{* \prime}+\boldsymbol{b}^{\dagger \prime}\right)}\right), \quad \overline{\theta^{\dagger}} \approx \overline{\theta^{*}}+\frac{1}{2} \overline{\boldsymbol{\zeta} \cdot \boldsymbol{\nabla}\left(\theta^{*^{\prime}}+\theta^{\dagger \prime}\right)}
$$

Here, to the order of accuracy attempted by Braginsky (1964a,b), we have $\boldsymbol{b}^{* \prime} \simeq \boldsymbol{\nabla} \times\left(\boldsymbol{\zeta} \times \overline{\boldsymbol{b}^{*}}\right)$, $\theta^{* \prime} \simeq-\boldsymbol{\zeta} \cdot \boldsymbol{\nabla} \overline{\theta^{*}}$ (see $\left.(19 \mathrm{a}, \mathrm{c})\right)$ and $\widetilde{\boldsymbol{b}}^{\dagger \prime} \simeq \mathbf{0}, \theta^{\dagger \prime} \simeq \mathbf{0}$. So with diffusion effects ignored, (197a,b) provides an explicit recipe linking the glm-values of $\overline{\boldsymbol{b}^{\dagger}}=\overline{\boldsymbol{b}}, \overline{\theta^{\dagger}}=\bar{\theta}$ to the Eulerian means $\overline{\boldsymbol{b}^{*}}, \overline{\theta^{*}}$. However, with the small diffusion effects included, we have $\boldsymbol{b}^{* \prime}+\boldsymbol{b}^{\dagger \prime} \neq \boldsymbol{\nabla} \times\left(\boldsymbol{\zeta} \times \overline{\boldsymbol{b}^{*}}\right)$, $\theta^{* \prime}+\theta^{\dagger \prime} \neq-\boldsymbol{\zeta} \cdot \nabla \overline{\theta^{*}}$. This means that, having solved the glm-equations for $\overline{\boldsymbol{b}}, \bar{\theta}$, one can only achieve the same $\mathrm{O}\left(\eta^{2} R m^{-1}\right), \mathrm{O}\left(\eta^{2} P e^{-1}\right)$ accuracy for $\overline{\boldsymbol{b}^{*}}, \overline{\theta^{*}}$ after obtaining the diffusive corrections $\mathrm{O}\left(\eta R m^{-1}\right), \mathrm{O}\left(\eta P e^{-1}\right)$ to $\boldsymbol{b}^{* \prime}, \theta^{* \prime}$. Put another way, without solving for the diffusive contributions to the Eulerian fluctuations, the glm-values are known to a greater order of accuracy than the Eulerian mean counterparts.

Though the large $R m$ nearly axisymmetric dynamo has a long and detailed history, the HEL-development continues to throw up some surprises, such as the above. Another surprise relates to the coordinate system. For though the HEL-idea is reasonably clear, the technical details of the cylindrical polar coordinate development of Soward (1972) need time and effort to follow. For that reason basic accounts, such as that given by Moffatt (1978) bypass those complications and explain the concepts within the simpler framework of Cartesian coordinates. Indeed Part I and Roberts and Soward (2009) made that simplification, as well as in their ETHEL-continuation (Soward and Roberts 2010). In this paper, we have highlighted the key points of Soward (1972) and demonstrated how they can be derived directly from the Cartesian form of Part I. We emphasise that the HEL-approach used by Soward was restricted to isochoric displacements $\mathcal{J}=1$, which as he noted (Soward 1972, eq. (4.24)) implied $\overline{\boldsymbol{\xi}}=$ 
$\mathrm{O}\left(\xi^{2}\right) \neq \mathbf{0}$ corresponding to the ETHEL mean consequence (195d). Our investigations here, particularly in section 5 and Appendix G, are based on $\mathcal{J}=1$ and the glm-postulate $\overline{\boldsymbol{\eta}}=\mathbf{0}$ (195c).

A matter that deserves comment is that turbulent dynamo theories often argue that the mean electromotive force caused by the turbulent fluctuations on a mean magnetic field $\bar{b}$ leads to a mean EMF that can be expressed in the form

$$
\overline{\alpha_{i j}} \overline{b_{j}}-\overline{\beta_{i j k}} \nabla_{j} \overline{b_{k}} \text {. }
$$

Then, in the case of isotropic turbulence, for which

$$
\overline{\alpha_{i j}}=\alpha \delta_{i j}, \quad \overline{\beta_{i j k}}=\beta \varepsilon_{i j k},
$$

one may refer to an $\alpha$-effect of strength $\alpha$ and a turbulent diffusivity of strength $\beta$. In the spirit of that approach, we can identify from (118) and (119) the mean EMF

$$
\mathcal{E}_{i} \equiv-\mathfrak{K} \mathrm{J}_{i} \doteq \mathfrak{K}\left(\overline{\alpha_{i j}} \overline{b_{j}}-\varepsilon_{i j k}\left(\overline{g_{j l}}-\delta_{j l}\right) \nabla_{l} \overline{b_{k}}\right),
$$

in which $\mathfrak{K} \overline{\alpha_{i j}}$ and $\mathfrak{K}\left(\overline{g_{j l}}-\delta_{j l}\right)$ could be interpreted as the $\alpha$-tensor and the turbulent magnetic diffusivity-tensor. The corresponding cylindrical polar coordinate form of the mean EMF (200a) follows directly from (164) but, because $(\boldsymbol{\nabla} \boldsymbol{b})^{p q}=\nabla^{p} b^{q}+s^{-1} \delta^{p 2} \epsilon^{3 r q} b^{r}$ (see (145b)), it possesses the distinct structure

$$
\mathcal{E}^{p} \equiv-\mathfrak{K} \mathrm{J}^{p} \doteq \mathfrak{K}\left(s^{-1} \overline{\aleph^{p q}} \overline{b^{q}}-\varepsilon^{p q t}\left(\overline{g^{q s}}-\delta^{q s}\right) \nabla^{s} \overline{b^{t}}\right)
$$

(see (158c) and (167a)), in which $\aleph^{p q}$ not only depends on $\boldsymbol{\alpha}$ but also contains additional terms originating from $\boldsymbol{g}$. The point here is that from the general tensor point of view, neither $\nabla^{s} \bar{b}^{t}$ nor $\aleph^{p q}$ are tensors. This is a matter that is clear from the general tensor development of Roberts and Soward (2006b). Indeed, the equations (163a,b) for the mean magnetic vector potential $a \widehat{\phi}$ and magnetic field $\overline{b_{\phi}} \widehat{\phi}$ reveal via (162c) that the $\alpha$-effect processes depend on yet a new symmetric matrix $\overline{\gamma^{p q}}$ defined by (169b). This is not special to the Braginsky dynamo but will happen whenever the coefficients in (198) are spatially varying and depends only on the use of a toroidal-poloidal decomposition of the mean magnetic induction equation.

A feature emphasised by $(200 \mathrm{a}, \mathrm{b})$ is that the turbulent $\alpha$ and diffusivity coefficients are all proportional to the magnetic diffusivity $\mathfrak{K}$. This means that any dynamo growth must depend on $\mathfrak{K}$ and so in the limit $R m \rightarrow \infty$ the growth rate based on the convective time $\mathcal{L} / \mathcal{U}$ tends to zero, i.e. it is by necessity a 'slow' rather than 'fast' dynamo (see, for example, Childress and Gilbert 1995).

The mechanism by which the Braginsky dynamo works, namely the diffusion of its primary magnetic field (here $\overline{b_{\phi}}$ ) to create a weak secondary magnetic field (here $\overline{\boldsymbol{b}_{\mathrm{m}}}$ ) upon which a shear flow can act to bootstrap the primary magnetic field, belongs to a class of slow dynamos discussed by Soward (1990). A prominent kinematic dynamo in that class is the screw dynamo of Ruzmaikin et al. (1988), which is the Ponomarenko (1973) dynamo with its discontinuous steady flow smoothed out leaving an axisymmetric steady spiralling flow; each cylinder $s=$ constant moves as a solid surface rotating about and translating along the $z$-axis. Though the primary field lines of the fastest growing screw dynamo modes lie on those cylindrical surfaces, they are not aligned to the primary streamlines, a possibility which is allowed by the rigid motion of the surfaces. This feature emphasises the importance of the structure of the magnetic field rather than the structure of the flow, an idea anticipated by the origins (185) and $(186 \mathrm{a}, \mathrm{b})$ of the Braginsky dynamo $\alpha$-effect.

More interestingly, the large Rm Archontis dynamo (Archontis 2000, Dorch and Archontis 2004), which is a fully hydromagnetic dynamo driven by a steady spatially periodic body force $\boldsymbol{F}^{*}$ (see also Gilbert et al. 2011, and references therein), may possibly operate the same way as the Braginsky dynamo. We use the qualification 'possibly' cautiously, because, though 
the magnetic field and flow are remarkably aligned, the existence of chaotic particle paths leaves open the possibility of other dynamo mechanisms, which are absent in the cases of the Braginsky and screw dynamos.

\section{Acknowledgments}

As in Soward and Roberts (2010), we thank Andrew Gilbert for drawing our attention to the fact that our ETHEL-development of section 3 relies on the well known concept in differential geometry of Lie dragging, which pivots on the Lie derivative (A.5). One of us (PHR) is grateful to the National Science Foundation for support under grant EAR-0911004.

\section{References}

Archontis, V.D., Study of generation and evolution of magnetic fields in stars using 3D MHD simulations of turbulent flows. PhD Thesis, Copenhagen University, 2000.

Andrews, D.G. and McIntyre, M.E., An exact theory of nonlinear waves on a Lagrangian-mean flow. J. Fluid Mech. 1978a, 89, 609-646.

Andrews, D.G. and McIntyre, M.E., On wave action and its relatives, J. Fluid Mech. 1978b, 89, 647-664: corrigendum, ibid, 1978, 95, 796.

Braginsky, S.I., Self-excitation of magnetic field during the motion of a highly conducting fluid, JETP 1964a, 47, 1084-1098. [Engl. transl. Sov. Phys. JETP 20, 726-735 (1965).]

Braginsky, S.I., Theory of the hydromagnetic dynamo, JETP 1964b, 47, 2178-2193. [Engl. transl. Sov. Phys. JETP 20, 1462-1471 (1965).]

Bühler, O., Waves and Mean Flows, Cambridge Monographs on Mechanics, 2009 (CUP, Cambridge).

Childress, S. and Gilbert, A., Stretch, Twist, and Fold: The Fast Dynamo, 1995 (Springer-Verlag, Berlin).

Cupal, I., Secondary $\alpha$-effect in the nearly symmetric dynamo. Astron. Nachr. 1984, 305, 297-299.

d'Inverno, R., Introducing Einstein's Relativity, 1992 (Clarendon Press, Oxford).

Dorch, S.B.F. and Archontis, V.D., On the saturation of astrophysical dynamos: Numerical experiments with the no-cosines flow. Solar Phys. 2004, 224, 171-178.

Eckart, C., Some transformations of the hydrodynamic equations. Phys. Fluids 1963, 6, 1037-1041.

Frieman, E. and Rotenberg, M., On hydromagnetic stability of stationary equilibria. Rev. Mod. Phys. 1960, 32, 898-902.

Gilbert, A.D., Ponty, Y. and Zheligovsky, V., Dissipative structures in a nonlinear dynamo. Geophys. Astrophys. Fluid Dyn. 2011, 105, 629-653.

Gubbins, G., Barber, C.N., Gibbons, S. and Love, J.J., Kinematic dynamo action in a sphere. I Effects of differential rotation and meridional circulation on solutions with axial dipole symmetry. Proc. R. Soc. Lond. A 2000, 32, 898-902.

Hawking, S.W. and Ellis, G.F.R. The Large Scale Structure of Space-Time, 1973 (CUP, Cambridge).

Holm, D.D., Averaged Lagrangians and mean effects of fluctuations in ideal fluid dynamics. Physica D 2002, 170, 253-286.

Kumar, S. and Roberts, A three-dimensional kinematic dynamo. Proc. R. Soc. Lond. A 1975, 344, 235-258.

McIntyre, M.E., Towards a Lagrangian-mean description of stratospheric circulations and chemical transports. Phil. Trans. R. Soc. Lond. A 1980, 296, 129-148.

Moffatt, H.K., Magnetic Field Generation in Electrically Conducting Fluids, Cambridge Monographs on Mechanics and Applied Mathematics, 1978 (CUP, Cambridge).

Moffatt, H.K., Magnetostatic equilibria and analogous Euler flows of arbitrary complex topology. Part 2. Stability considerations. J. Fluid Mech. 1986, 166, 359-378.

Ponomarenko, Y.B., On the theory of hydromagnetic dynamos. Zh. Prikl. Mekh and Tekh. Fiz. (USSR) 1973, 6, 47-51.

Roberts, P.H. and Aurnou, J.M., On the theory of core-mantle coupling. Geophys. Astrophys. Fluid Dyn. 2012, 106, $157-230$

Roberts, P.H. and Soward, A.M., Eulerian and Lagrangian means in rotating, magnetohydrodynamic flows I. General results. Geophys. Astrophys. Fluid Dyn. 2006a, 100, 457-483.

Roberts, P.H. and Soward, A.M., Covariant description of non-relativistic magnetohydrodynamics. Geophys. Astrophys. Fluid Dyn. 2006b, 100, 485-502.

Roberts, P.H. and Soward, A.M., The Navier-Stokes-alpha equations revisited. Geophys. Astrophys. Fluid Dyn. 2009, 103, 303-316.

Ruzmaikin, A., Sokoloff, D. and Shukurov, A., Hydromagnetic screw dynamo. J. Fluid Mech. 1988, 197, 39-56.

Sarson, G. and Gubbins, D., Three-dimensional kinematic dynamos dominated by strong differential rotation. J. Fluid Mech. 1996, 306, 223-265.

Schutz, B., Geometrical Methods of Mathematical Physics, 1980 (CUP, Cambridge).

Soward, A. M., Nearly symmetric kinematic and hydromagnetic dynamos. J. Math. Phys. 1971a, 12, 1900-1906. 
Soward, A. M., Nearly symmetric advection. J. Math. Phys. 1971b, 12, 2052-2062.

Soward, A.M., A kinematic theory of large magnetic Reynolds number dynamos. Phil. Trans. R. Soc. Lond. A 1972, 272, 431-462.

Soward, A. M., A unified approach to a class of slow dynamos. Geophys. Astrophys. Fluid Dyn. 1990, 53, 81-107.

Soward, A.M. and Roberts, P.H., On the derivation of the Navier-Stokes-alpha equations from Hamilton's Principle. J. Fluid Mech. 2008, 604, 297-323.

Soward, A.M. and Roberts, P.H., The hybrid Euler-Lagrange procedure using an extension of Moffatt's method. J. Fluid Mech. 2010, 661, 45-72.

Taylor, J.B., The magnetohydrodynamics of a rotating fluid and the Earth's dynamo problem. Proc. R. Soc. Lond. A 1963, 274, 274-283.

Tough, J.G., Nearly symmetric dynamos. Geophys. J. R. Astr. Soc. 1967, 13, 393-369.

Tough, J.G. and Roberts, P.H., Nearly symmetric hydromagnetic dynamos. Phys. Earth Planet. Inter. 1968, 1, 288-296.

Vladimirov, V.A., Magnetohydrodynamic drift equations: from langmuir circulation to magneto hydrodynamic dynamo? J. Fluid Mech. 2012, 698, 51-61.

\section{Appendix A: General tensors and the Lie derivative}

As a preliminary to the construction of Lie derivatives we note that, for any vectors $\boldsymbol{w}$ and $\boldsymbol{w}^{*}$ related by $\boldsymbol{w}^{* L}=\boldsymbol{w} \cdot \boldsymbol{\nabla} \boldsymbol{x}^{L}$ and $\boldsymbol{w}=\boldsymbol{w}^{* L} \cdot \boldsymbol{\nabla}^{L} \boldsymbol{x}$ as in (61a) and (62a) (not just $\boldsymbol{w}^{* L}=\partial_{t} \boldsymbol{x}^{L}$ ), we have

$$
\begin{aligned}
\left(\boldsymbol{\nabla} \boldsymbol{x}^{L}\right) \cdot\left(\boldsymbol{\nabla} \boldsymbol{w}^{*}\right)^{L} & =\boldsymbol{w} \cdot \boldsymbol{\nabla}\left(\boldsymbol{\nabla} \boldsymbol{x}^{L}\right)+(\boldsymbol{\nabla} \boldsymbol{w}) \cdot\left(\boldsymbol{\nabla} \boldsymbol{x}^{L}\right), \\
-\left(\boldsymbol{\nabla} \boldsymbol{w}^{*}\right)^{L} \cdot\left(\boldsymbol{\nabla}^{L} \boldsymbol{x}\right) & =\boldsymbol{w} \cdot \boldsymbol{\nabla}\left(\boldsymbol{\nabla}^{L} \boldsymbol{x}\right)-\left(\boldsymbol{\nabla}^{L} \boldsymbol{x}\right) \cdot(\boldsymbol{\nabla} \boldsymbol{w}), \\
\mathcal{J}\left(\boldsymbol{\nabla} \cdot \boldsymbol{w}^{*}\right)^{L} & =\boldsymbol{w} \cdot \boldsymbol{\nabla} \mathcal{J}+\mathcal{J}(\boldsymbol{\nabla} \cdot \boldsymbol{w}) .
\end{aligned}
$$

Here (A.1a) results from consideration of $\boldsymbol{\nabla} \boldsymbol{w}^{* L}=\boldsymbol{\nabla}\left(\boldsymbol{w} \cdot \boldsymbol{\nabla} \boldsymbol{x}^{L}\right)$, (A.1b) follows from consideration of $\boldsymbol{w} \cdot \boldsymbol{\nabla}\left(\left(\boldsymbol{\nabla} \boldsymbol{x}^{L}\right)\left(\boldsymbol{\nabla}^{L} \boldsymbol{x}\right)\right)=\boldsymbol{w} \cdot \boldsymbol{\nabla} \mathbf{I}=0$ (see (64a,b)), while (A.1c) is a reiteration of (75b).

In ths Appendix we determine how the time derivative of tensor quantities $\mathcal{T}^{*}$ (see (60)) transform under the mapping $\boldsymbol{x} \mapsto \boldsymbol{x}^{L}$. We begin by considering the gradient of $\boldsymbol{w}^{* L}=\partial_{t} \boldsymbol{x}^{L}$, namely (59b) which determines $\partial_{t}\left(\boldsymbol{\nabla} \boldsymbol{x}^{L}\right)=\boldsymbol{\nabla} \boldsymbol{w}^{* L}=\left(\boldsymbol{\nabla} \boldsymbol{x}^{L}\right) \cdot\left(\boldsymbol{\nabla} \boldsymbol{w}^{*}\right)^{L}$. From it and (A.1a,b), we deduce

$$
\begin{array}{r}
\partial_{t}\left(\boldsymbol{\nabla} \boldsymbol{x}^{L}\right)=\left(\boldsymbol{\nabla} \boldsymbol{x}^{L}\right) \cdot\left(\boldsymbol{\nabla} \boldsymbol{w}^{*}\right)^{L}=\boldsymbol{w} \cdot \boldsymbol{\nabla}\left(\boldsymbol{\nabla} \boldsymbol{x}^{L}\right)+(\boldsymbol{\nabla} \boldsymbol{w}) \cdot\left(\boldsymbol{\nabla} \boldsymbol{x}^{L}\right), \\
\partial_{t}\left(\boldsymbol{\nabla}^{L} \boldsymbol{x}\right)=-\left(\boldsymbol{\nabla} \boldsymbol{w}^{*}\right)^{L} \cdot\left(\boldsymbol{\nabla}^{L} \boldsymbol{x}\right)=\boldsymbol{w} \cdot \boldsymbol{\nabla}\left(\boldsymbol{\nabla}^{L} \boldsymbol{x}\right)-\left(\boldsymbol{\nabla}^{L} \boldsymbol{x}\right) \cdot(\boldsymbol{\nabla} \boldsymbol{w}),
\end{array}
$$

where, in deriving (A.2b), we have used the identity $\partial_{t}\left(\left(\boldsymbol{\nabla} \boldsymbol{x}^{L}\right) \cdot\left(\boldsymbol{\nabla}^{L} \boldsymbol{x}\right)\right)=\partial_{t} \mathbf{I}=\mathbf{0}$. Differentiation of $\mathcal{J} \epsilon_{i j k}^{*}$ defined by (70a) and use of (A.2a) determines

$$
\epsilon_{i j k}^{*} \frac{\partial \mathcal{J}}{\partial t}=\mathcal{J}\left(\epsilon_{l j k}^{*} \nabla_{l}^{L} w_{i}^{* L}+\epsilon_{i l k}^{*} \nabla_{l}^{L} w_{j}^{* L}+\epsilon_{i j l}^{*} \nabla_{l}^{L} w_{k}^{* L}\right)=\mathcal{J} \epsilon_{i j k}^{*}\left(\nabla \cdot \boldsymbol{w}^{*}\right)^{L}
$$

by (B.1d) below, each component of which yields

$$
\partial_{t} \mathcal{J}=\mathcal{J}\left(\boldsymbol{\nabla} \cdot \boldsymbol{w}^{*}\right)^{L}=\boldsymbol{w} \cdot \boldsymbol{\nabla} \mathcal{J}+\mathcal{J} \boldsymbol{\nabla} \cdot \boldsymbol{w}
$$

with the help of (A.1c).

The above ideas may be extended to tensor quantities. For clarity, we briefly digress into general tensor notation. We distinguish contravariant (upper index), covariant (lower index) tensors and write

$$
\left(\mathcal{T}_{E \cdots G}^{* A \cdots D}\right)^{L}=\mathcal{J}^{\mathrm{w}}\left(\nabla_{E}^{L} x_{e}\right) \cdots\left(\nabla_{G}^{L} x_{g}\right) \mathcal{T}_{e \cdots g}^{a \cdots d}\left(\nabla_{a} x_{A}^{L}\right) \cdots\left(\nabla_{d} x_{D}^{L}\right)
$$


for mixed tensors (upper and lower indices) of weight w. Then direct use of (76a) yields

$$
\begin{aligned}
\left(\partial_{t} \mathcal{T}_{E \cdots G}^{* \cdots \cdots}\right)^{L} & =\left(\partial_{t}-\boldsymbol{w} \cdot \nabla\right)\left(\mathcal{J}^{\mathrm{w}}\left(\nabla_{E}^{L} x_{e}\right) \cdots\left(\nabla_{G}^{L} x_{g}\right) \mathcal{T}_{e \cdots g}^{a \cdots d}\left(\nabla_{a} x_{A}^{L}\right) \cdots\left(\nabla_{d} x_{D}^{L}\right)\right) \\
& =\mathcal{J}^{\mathrm{w}}\left(\nabla_{E}^{L} x_{e}\right) \cdots\left(\nabla_{G}^{L} x_{g}\right)\left(\partial_{t} \mathcal{T}_{e \cdots g}^{a \cdots d}-\left(\mathrm{L}_{\boldsymbol{w}} \mathcal{T}\right)_{e \cdots g}^{a \cdots d}\right)\left(\nabla_{a} x_{A}^{L}\right) \cdots\left(\nabla_{d} x_{D}^{L}\right)
\end{aligned}
$$

with the help of (A.2a.b,d), or after inverting

$$
\mathcal{J}^{-\mathrm{w}}\left(\nabla_{e} x_{E}^{L}\right) \cdots\left(\nabla_{g} x_{G}^{L}\right)\left(\partial_{t} \mathcal{T}_{E \cdots G}^{* A \cdots D}\right)^{L}\left(\nabla_{A}^{L} x_{a}\right) \cdots\left(\nabla_{D}^{L} x_{d}\right)=\partial_{t} \mathcal{T}_{e \cdots g}^{a \cdots d}-\left(\mathrm{L}_{\boldsymbol{w}} \mathcal{T}\right)_{e \cdots g}^{a \cdots d},
$$

where $\mathrm{L}_{\boldsymbol{w}}$ is the Lie derivative defined by

$$
\begin{aligned}
\left(\mathrm{L}_{\boldsymbol{w}} \mathcal{T}\right)_{e f \cdots g}^{a b \cdots d} \equiv & \boldsymbol{w} \cdot \nabla \mathcal{T}_{e f \cdots g}^{a b \cdots d}-\mathcal{T}_{e f \cdots g}^{j b \cdots d}\left(\nabla_{j} w^{a}\right)-\text { (all upper indices) } \\
& -\mathrm{w}\left(\nabla_{j} w^{j}\right) \mathcal{T}_{e f \cdots g}^{a b \cdots d}+\left(\nabla_{e} w^{j}\right) \mathcal{T}_{j f \cdots g}^{a b \cdots d}+\text { (all lower indices) }
\end{aligned}
$$

(see Hawking and Ellis 1973, eq. (2.12) but generalised to include the tensor weight). Reassuringly the Lie derivatives of the unweighted mixed unit tensor $\mathbf{I}_{i}^{j}$, the contavariant tensor $\epsilon^{i j k}$ weight $\mathrm{w}=-1$ and the covariant tensor $\epsilon_{i j k}$ weight $\mathrm{w}=1$ (see $(70 \mathrm{a}, \mathrm{b})$ respectively) all vanish:

$$
\mathrm{L}_{\boldsymbol{w}} \mathbf{I}=\boldsymbol{w} \cdot \boldsymbol{\nabla} \mathbf{I}=\mathbf{0}, \quad \mathrm{L}_{\boldsymbol{w}} \boldsymbol{\epsilon}=\boldsymbol{w} \cdot \boldsymbol{\nabla} \boldsymbol{\epsilon}=\mathbf{0}
$$

on use of (B.1d) (cf. the time derivative of $\mathcal{J} \epsilon_{i j k}^{*}$ in (A.2c)), and since $\mathbf{I}$ and $\boldsymbol{\epsilon}$ are constant tensors.

For any contravariant vector $\boldsymbol{w}, \mathrm{L}_{\boldsymbol{w}} \boldsymbol{\mathcal { T }}$ transforms as a tensor of the same rank and weight as $\mathcal{T}$ in the usual way:

$$
\left(\left(\mathrm{L}_{\boldsymbol{w}^{*}} \mathcal{T}^{*}\right)_{E \cdots G}^{A \cdots D}\right)^{L}=\mathcal{J}^{\mathrm{w}}\left(\nabla_{E}^{L} x_{e}\right) \cdots\left(\nabla_{G}^{L} x_{g}\right)\left(\mathrm{L}_{\boldsymbol{w}} \mathcal{T}\right)_{e f \cdots g}^{a b \cdots d}\left(\nabla_{a} x_{A}^{L}\right) \cdots\left(\nabla_{d} x_{D}\right)
$$

a result readily established like (A.4a) but using (A.1a-c) rather than (A.2a,b,d).

With the notable exception of $\mathrm{I}_{i}^{j}=\delta_{i j}$, we do not invoke mixed tensors in this paper and so refrain from using the upper and lower index notation to indicate type. Instead we identify contravariant vectors by use of lower case symbols, e.g., $\mathcal{T}^{i}=u_{i}, b_{i}$ (also we write $w_{j}$ for $w^{j}$ ) and their covariant form by upper case symbols, e.g., $\mathcal{T}_{i}=V_{i}, H_{i}$. The main reason for doing this is that we wish to work in a Cartesian coordinate frame relative to which we wish to use the bold vector notation $\boldsymbol{u}, \boldsymbol{V}$ etc.

\section{Appendix B: The Lie derivatives of products}

We start by establishing some basic formulae. For the expression $\epsilon_{k l n} \epsilon_{n j o} \epsilon_{o m i}$ the contraction on the repeated subscript $o$ and the alternative contraction on the repeated subscript $n$ leads respectively to

$$
\epsilon_{k l n} \epsilon_{n j o} \epsilon_{o m i}=\left\{\begin{array}{l}
\epsilon_{k l m} \delta_{i j}-\epsilon_{k l i} \delta_{m j} \\
\delta_{k j} \epsilon_{l m i}-\delta_{l j} \epsilon_{k m i}
\end{array} .\right.
$$

Note that the superscript* used in Appendix A is superfluous in the Cartesian context of this Appendix (see, e.g., (70c)). The equivalence of the two right-had sides leads to the useful identity

$$
\delta_{i j} \epsilon_{k l m}=\delta_{k j} \epsilon_{i l m}+\delta_{l j} \epsilon_{k i m}+\delta_{m j} \epsilon_{k l i}
$$

which on contraction with $\nabla_{i} w_{j}$ gives

$$
\epsilon_{k l m} \boldsymbol{\nabla} \cdot \boldsymbol{w}=\epsilon_{n l m} \nabla_{n} w_{k}+\epsilon_{k n m} \nabla_{n} w_{l}+\epsilon_{k l n} \nabla_{n} w_{m} .
$$


On contracting the formulae (B.1a,b) with $\nabla_{j}$ we obtain

$$
\epsilon_{k l m} \nabla_{i}-\epsilon_{k l i} \nabla_{m}=\epsilon_{l m i} \nabla_{k}-\epsilon_{k m i} \nabla_{l} .
$$

As an example of its use, we consider its action on $\eta_{l}$ and then contract the resulting formula with $\nabla_{k} \eta_{m}$ to obtain

$$
\epsilon_{i k l}\left(\nabla_{k} \eta_{m}\right)\left(\nabla_{m} \eta_{l}\right)=(\boldsymbol{\nabla} \cdot \boldsymbol{\eta})(\boldsymbol{\nabla} \times \boldsymbol{\eta})_{i}-\left(\nabla_{i} \eta_{j}\right)(\boldsymbol{\nabla} \times \boldsymbol{\eta})_{j} .
$$

Another application of $(\mathrm{B} .1 \mathrm{a}, \mathrm{b})$ is the respective consequences

$$
\begin{aligned}
\boldsymbol{\zeta} \times & (\boldsymbol{\nabla} \times(\boldsymbol{u} \times \boldsymbol{b})) \\
& =\left\{\begin{array}{l}
-((\boldsymbol{b} \cdot \boldsymbol{\nabla}) \boldsymbol{u}) \times \boldsymbol{\zeta}+\boldsymbol{\zeta} \times \boldsymbol{u}(\boldsymbol{\nabla} \cdot \boldsymbol{b})-\boldsymbol{\zeta} \times((\boldsymbol{u} \cdot \boldsymbol{\nabla}) \boldsymbol{b})+\boldsymbol{b} \times \boldsymbol{\zeta}(\boldsymbol{\nabla} \cdot \boldsymbol{u}), \\
(\boldsymbol{\nabla} \boldsymbol{b}) \cdot(\boldsymbol{\zeta} \times \boldsymbol{u})+(\boldsymbol{\nabla} \boldsymbol{u}) \cdot(\boldsymbol{b} \times \boldsymbol{\zeta})-(\boldsymbol{\zeta} \cdot \boldsymbol{\nabla})(\boldsymbol{u} \times \boldsymbol{b})
\end{array}\right.
\end{aligned}
$$

for arbitrary vectors $\boldsymbol{\zeta}, \boldsymbol{u}$ and $\boldsymbol{b}$. We write $\boldsymbol{\zeta} \times(\boldsymbol{\nabla} \times(\boldsymbol{u} \times \boldsymbol{b}))$ as half the sum (B.4a)+(B.4b). We then form the sum with the other two quantities given by cyclically permuting $\boldsymbol{\zeta}, \boldsymbol{u}$ and $\boldsymbol{b}$ and so obtain

$$
\begin{aligned}
\boldsymbol{\zeta} \times(\boldsymbol{\nabla} \times(\boldsymbol{u} \times \boldsymbol{b}))+\boldsymbol{u} \times(\boldsymbol{\nabla} \times(\boldsymbol{b} \times \boldsymbol{\zeta}))+\boldsymbol{b} \times(\boldsymbol{\nabla} \times(\boldsymbol{\zeta} \times \boldsymbol{u})) \\
=(\boldsymbol{\nabla} \cdot \boldsymbol{\zeta}) \boldsymbol{u} \times \boldsymbol{b}+(\boldsymbol{\nabla} \cdot \boldsymbol{u}) \boldsymbol{b} \times \boldsymbol{\zeta}+(\boldsymbol{\nabla} \cdot \boldsymbol{b}) \boldsymbol{\zeta} \times \boldsymbol{u}+\boldsymbol{\nabla}(\boldsymbol{\zeta} \cdot(\boldsymbol{u} \times \boldsymbol{b})),
\end{aligned}
$$

which in view of the definitions (10a-c) may be expressed succinctly in the form

$$
\boldsymbol{u} \times \llbracket \boldsymbol{\zeta}, \boldsymbol{b} \rrbracket+[\boldsymbol{\zeta}, \boldsymbol{u}] \times \boldsymbol{b}=\{\boldsymbol{\zeta},(\boldsymbol{u} \times \boldsymbol{b})\} .
$$

When $\boldsymbol{b}$ is solenoidal, (B.5b) may be expressed, on reversing the signs, in the alternative form

$$
\boldsymbol{u} \times(\boldsymbol{\nabla} \times(\boldsymbol{\zeta} \times \boldsymbol{b}))-[\boldsymbol{\zeta}, \boldsymbol{u}] \times \boldsymbol{b}=-\{\boldsymbol{\zeta},(\boldsymbol{u} \times \boldsymbol{b})\} \quad(\boldsymbol{\nabla} \cdot \boldsymbol{b}=0) .
$$

The formula (10a) for $[\boldsymbol{\zeta}, \boldsymbol{u}]$ has a useful generalisation obtained upon multiplying it by the arbitrary scalar $\rho$ :

$$
\begin{aligned}
\rho[\boldsymbol{\zeta}, \boldsymbol{u}]+(\boldsymbol{\nabla} \cdot(\rho \boldsymbol{\zeta})) \boldsymbol{u} & =[\boldsymbol{\zeta}, \rho \boldsymbol{u}]+(\boldsymbol{\nabla} \cdot \boldsymbol{\zeta}) \rho \boldsymbol{u}=\llbracket \boldsymbol{\zeta},(\rho \boldsymbol{u}) \rrbracket \\
& =-\boldsymbol{\nabla} \times(\rho(\boldsymbol{\zeta} \times \boldsymbol{u}))+(\boldsymbol{\nabla} \cdot(\rho \boldsymbol{u})) \boldsymbol{\zeta}
\end{aligned}
$$

(see $(10 \mathrm{~b}))$. The scalar product of $[\boldsymbol{\zeta}, \boldsymbol{u}]$ with the arbitrary gradient $\boldsymbol{\nabla} \theta$ yields

$$
[\boldsymbol{\zeta}, \boldsymbol{u}] \cdot \boldsymbol{\nabla} \theta+\boldsymbol{u} \cdot \boldsymbol{\nabla}(\boldsymbol{\zeta} \cdot \boldsymbol{\nabla} \theta)=\boldsymbol{\zeta} \cdot \boldsymbol{\nabla}(\boldsymbol{u} \cdot \boldsymbol{\nabla} \theta) .
$$

The results (B.5b), (B.7a) and (B.8) have the succinct interpretation as the Lie derivative of products:

$$
\begin{aligned}
\mathrm{L}_{\boldsymbol{\zeta}}(\boldsymbol{u} \times \boldsymbol{b}) & =\boldsymbol{u} \times\left(\mathrm{L}_{\boldsymbol{\zeta}} \boldsymbol{b}\right)+\left(\mathrm{L}_{\zeta} \boldsymbol{u}\right) \times \boldsymbol{b}, \\
\mathrm{L}_{\boldsymbol{\zeta}}(\rho \boldsymbol{u}) & =\left(\mathrm{L}_{\boldsymbol{\zeta}} \rho\right) \boldsymbol{u}+\rho\left(\mathrm{L}_{\boldsymbol{\zeta}} \boldsymbol{u}\right), \\
\mathrm{L}_{\boldsymbol{\zeta}}(\boldsymbol{u} \cdot \nabla \theta) & =\boldsymbol{u} \cdot\left(\mathrm{L}_{\boldsymbol{\zeta}}(\boldsymbol{\nabla} \theta)\right)+\left(\mathrm{L}_{\boldsymbol{\zeta}} \boldsymbol{u}\right) \cdot \nabla \theta,
\end{aligned}
$$

where the Lie derivative $\mathrm{L}_{\zeta}$ is defined for each of the scalars and vectors of various weights appearing in (B.9a-c) by (99a-e), under the change of variable $\boldsymbol{\eta} \mapsto \boldsymbol{\zeta}$. Respectively, the results $(\mathrm{B} .9 \mathrm{c}, \mathrm{a})$ are made plausible because the Lie derivatives of $\mathbf{I}$ and $\boldsymbol{\epsilon}$, that realise the scalar and vector products, vanish. The former $\mathrm{L}_{\boldsymbol{\zeta}} \mathbf{I}=\mathbf{0}$ (see (A.6a)) is trivial, while the latter $\mathrm{L}_{\boldsymbol{\zeta}} \boldsymbol{\epsilon}=\mathbf{0}$ (see (A.6b)) depends on the identity (B.1d).

\section{Appendix C: Perfect fluids}

We derive the formulae for fluctuating and mean quantities reported in section 1.2 for the perfect fluid case, $\mathfrak{K}=0, \kappa=0, q^{*}=0$ in Appendices C.1 and C.2 that follow, under the 
small $\zeta$ assumption (12).

\section{C.1 The Frieman and Rotenberg (1960) representation of $b^{* \prime}, \rho^{* \prime}$ and $\theta^{* \prime}$ at $\mathrm{O}(\zeta)$}

Though the representation of the fluctuations $\boldsymbol{b}^{* \prime}, \rho^{* \prime}$ and $\theta^{* \prime}$ at $\mathrm{O}(\zeta)$ is well known, the techniques, that we use here are readily adapted to the more involved applications in the subsequent Appendices C.2 and D.

- Fluctuating magnetic field, $\boldsymbol{b}^{* \prime}$. Noting that $\boldsymbol{u}^{* \prime}=\partial_{t} \boldsymbol{\zeta}-\left[\boldsymbol{\zeta}, \overline{\boldsymbol{u}^{*}}\right]$ (see (9a)), we may utilise the formula (B.6), because $\boldsymbol{\nabla} \cdot \overline{\boldsymbol{b}^{*}}=0$, to obtain

$$
\begin{aligned}
\overline{\boldsymbol{u}^{*}} \times\left(\boldsymbol{\nabla} \times\left(\boldsymbol{\zeta} \times \overline{\boldsymbol{b}^{*}}\right)\right)+\boldsymbol{u}^{* \prime} \times \overline{\boldsymbol{b}^{*}} & =\left(\partial_{t} \boldsymbol{\zeta}\right) \times \overline{\boldsymbol{b}^{*}}-\left\{\boldsymbol{\zeta},\left(\boldsymbol{\nabla} \times\left(\overline{\boldsymbol{u}^{*}} \times \overline{\boldsymbol{b}^{*}}\right)\right)\right\} \\
& \approx \partial_{t}\left(\boldsymbol{\zeta} \times \overline{\boldsymbol{b}^{*}}\right)-\nabla\left(\boldsymbol{\zeta} \cdot\left(\overline{\boldsymbol{u}^{*}} \times \overline{\boldsymbol{b}^{*}}\right)\right),
\end{aligned}
$$

since $\partial_{t} \overline{\boldsymbol{b}^{*}} \simeq \boldsymbol{\nabla} \times\left(\overline{\boldsymbol{u}^{*}} \times \overline{\boldsymbol{b}^{*}}\right)($ see $(22 \mathrm{a}, \mathrm{b}))$. Comparison with $\overline{\boldsymbol{u}^{*}} \times \boldsymbol{b}^{* \prime}+\boldsymbol{u}^{* \prime} \times \overline{\boldsymbol{b}^{*}} \simeq-\boldsymbol{E}^{* \prime}$ (see (16b)) verifies that $\boldsymbol{b}^{* \prime} \simeq \boldsymbol{\nabla} \times\left(\boldsymbol{\zeta} \times \overline{\boldsymbol{b}^{*}}\right)($ see $(19 \mathrm{a}))$ and $\boldsymbol{E}^{* \prime} \simeq-\partial_{t}\left(\boldsymbol{\zeta} \times \overline{\boldsymbol{b}^{*}}\right)+\boldsymbol{\nabla}\left(\boldsymbol{\zeta} \cdot\left(\overline{\boldsymbol{u}^{*}} \times \overline{\boldsymbol{b}^{*}}\right)\right)$ (see $(20 \mathrm{a}, \mathrm{b}))$ solves $\partial_{t} \boldsymbol{b}^{* \prime}=-\boldsymbol{\nabla} \times \boldsymbol{E}^{* \prime}$ (see (16a)).

- Fluctuating density, $\rho^{* \prime}$. Likewise (B.7a,b) yield

$$
\begin{aligned}
-\left(\boldsymbol{\nabla} \cdot\left(\overline{\rho^{*}} \boldsymbol{\zeta}\right)\right) \overline{\boldsymbol{u}^{*}}+\overline{\rho^{*}} \boldsymbol{u}^{* \prime} & =\overline{\rho^{*}} \partial_{t} \boldsymbol{\zeta}-\llbracket \boldsymbol{\zeta},\left(\overline{\rho^{*}} \overline{\boldsymbol{u}^{*}}\right) \rrbracket \\
& \approx \partial_{t}\left(\overline{\rho^{*} \boldsymbol{\zeta}}\right)+\boldsymbol{\nabla} \times\left(\boldsymbol{\zeta} \times\left(\overline{\rho^{*}} \overline{\boldsymbol{u}^{*}}\right)\right),
\end{aligned}
$$

since $\partial_{t} \overline{\rho^{*}} \simeq-\boldsymbol{\nabla} \cdot\left(\overline{\rho^{*}} \overline{\boldsymbol{u}^{*}}\right)($ see $(23 \mathrm{a}, \mathrm{b}))$. Comparison with $\rho^{* \prime} \overline{\boldsymbol{u}^{*}}+\overline{\rho^{*}} \boldsymbol{u}^{* \prime} \simeq \boldsymbol{m}^{* \prime}$ (see $\left.(17 \mathrm{~b})\right)$ verifies that $\rho^{* \prime} \simeq-\boldsymbol{\nabla} \cdot\left(\overline{\rho^{*} \boldsymbol{\zeta}}\right)$ (see $\left.(19 \mathrm{~b})\right)$ and $\boldsymbol{m}^{* \prime} \simeq \partial_{t}\left(\overline{\rho^{*} \boldsymbol{\zeta}}\right)+\boldsymbol{\nabla} \times\left(\boldsymbol{\zeta} \times\left(\overline{\rho^{*}} \overline{\boldsymbol{u}^{*}}\right)\right)$ (see $(20 \mathrm{c}, \mathrm{d})$ solves $\partial_{t} \rho^{* \prime}=-\nabla \cdot \boldsymbol{m}^{* \prime}$ (see $\left.(17 \mathrm{a})\right)$.

- Fluctuating temperature, $\theta^{* \prime}$. Similarly (B.8) gives

$$
\begin{aligned}
-\overline{\boldsymbol{u}^{*}} \cdot \boldsymbol{\nabla}\left(\boldsymbol{\zeta} \cdot \boldsymbol{\nabla} \overline{\theta^{*}}\right)+\boldsymbol{u}^{* \prime} \cdot \boldsymbol{\nabla} \overline{\theta^{*}} & =\left(\partial_{t} \boldsymbol{\zeta}\right) \cdot \boldsymbol{\nabla} \overline{\theta^{*}}-\boldsymbol{\zeta} \cdot \boldsymbol{\nabla}\left(\overline{\boldsymbol{u}^{*}} \cdot \boldsymbol{\nabla} \overline{\theta^{*}}\right) \\
& \approx \partial_{t}\left(\boldsymbol{\zeta} \cdot \boldsymbol{\nabla} \overline{\theta^{*}}\right),
\end{aligned}
$$

since $\partial_{t} \overline{\theta^{*}} \simeq-\overline{\boldsymbol{u}^{*}} \cdot \boldsymbol{\nabla} \overline{\theta^{*}}($ see $(24))$. The identity shows that $\overline{\boldsymbol{u}^{*}} \cdot \boldsymbol{\nabla} \theta^{* \prime}+\boldsymbol{u}^{* \prime} \cdot \boldsymbol{\nabla} \overline{\theta^{*}} \simeq-\partial_{t} \theta^{* \prime}$ (see $(18))$ is solved by $\theta^{* \prime} \simeq-\boldsymbol{\zeta} \cdot \boldsymbol{\nabla} \overline{\theta^{*}}($ see $(19 \mathrm{c}))$.

\section{C.2 The effective-variables $u^{\dagger}, b^{\dagger}, \rho^{\dagger}$ and $\theta^{\dagger}$ at $\mathrm{O}\left(\zeta^{2}\right)$}

We slightly modify the arguments of Appendix C.1 to develop forms for the effective-variables $\boldsymbol{u}^{\dagger}, \boldsymbol{b}^{\dagger}, \rho^{\dagger}$ and $\theta^{\dagger}$ at $\mathrm{O}\left(\zeta^{2}\right)$.

- Effective magnetic field, $\boldsymbol{b}^{\dagger}$. We utilise $\boldsymbol{b}^{* \prime} \simeq \boldsymbol{\nabla} \times\left(\boldsymbol{\zeta} \times \overline{\boldsymbol{b}^{*}}\right)$, in addition to $\boldsymbol{u}^{* \prime}=\partial_{t} \boldsymbol{\zeta}-\left[\boldsymbol{\zeta}, \overline{\boldsymbol{u}^{*}}\right]$, and consider the sum $\left(\boldsymbol{u}^{* \prime}-\partial_{t} \boldsymbol{\zeta}\right) \times \boldsymbol{b}^{* \prime}+\boldsymbol{u}^{* \prime} \times\left(\nabla \times\left(\boldsymbol{\zeta} \times \overline{\boldsymbol{b}^{*}}\right)\right)$ :

$$
2 \boldsymbol{u}^{* \prime} \times \boldsymbol{b}^{* \prime}-\left(\partial_{t} \boldsymbol{\zeta}\right) \times \boldsymbol{b}^{* \prime} \approx-\left[\boldsymbol{\zeta}, \overline{\boldsymbol{u}^{*}}\right] \times \boldsymbol{b}^{* \prime}+\boldsymbol{u}^{* \prime} \times\left(\boldsymbol{\nabla} \times\left(\boldsymbol{\zeta} \times \overline{\boldsymbol{b}^{*}}\right)\right) .
$$

Noting that $\boldsymbol{\nabla} \cdot \overline{\boldsymbol{b}^{*}}=0$ and $\boldsymbol{\nabla} \cdot \boldsymbol{b}^{* \prime}=0$, we use (B.6) to obtain the alternative right-hand side

$$
\begin{aligned}
& =-\overline{\boldsymbol{u}^{*}} \times\left(\boldsymbol{\nabla} \times\left(\boldsymbol{\zeta} \times \boldsymbol{b}^{* \prime}\right)\right)-\left\{\boldsymbol{\zeta},\left(\overline{\boldsymbol{u}^{*}} \times \boldsymbol{b}^{* \prime}\right)\right\}+\left[\boldsymbol{\zeta}, \boldsymbol{u}^{*}\right] \times \overline{\boldsymbol{b}^{*}}-\left\{\boldsymbol{\zeta},\left(\boldsymbol{u}^{* \prime} \times \overline{\boldsymbol{b}^{*}}\right)\right\} \\
& \approx-\overline{\boldsymbol{u}^{*}} \times\left(\boldsymbol{\nabla} \times\left(\boldsymbol{\zeta} \times \boldsymbol{b}^{* \prime}\right)\right)+\left[\boldsymbol{\zeta}, \boldsymbol{u}^{* \prime}\right] \times \overline{\boldsymbol{b}^{*}}+\left\{\boldsymbol{\zeta}, \boldsymbol{E}^{* \prime}\right\},
\end{aligned}
$$

since $\boldsymbol{E}^{* \prime} \simeq-\overline{\boldsymbol{u}^{*}} \times \boldsymbol{b}^{* \prime}-\boldsymbol{u}^{* \prime} \times \overline{\boldsymbol{b}^{*}}$. Noting the identity $\left\{\boldsymbol{\zeta}, \boldsymbol{E}^{* \prime}\right\}=-\boldsymbol{\zeta} \times\left(\boldsymbol{\nabla} \times \boldsymbol{E}^{* \prime}\right)+\boldsymbol{\nabla}\left(\boldsymbol{\zeta} \cdot \boldsymbol{E}^{* \prime}\right)$ (see (10c)) and use of $\partial_{t} \boldsymbol{b}^{* \prime}=-\boldsymbol{\nabla} \times \boldsymbol{E}^{* \prime}$ enables us to reduce (C.4a,c) to

$$
2 \boldsymbol{u}^{* \prime} \times \boldsymbol{b}^{* \prime} \approx \partial_{t}\left(\boldsymbol{\zeta} \times \boldsymbol{b}^{* \prime}\right)-\overline{\boldsymbol{u}^{*}} \times\left(\boldsymbol{\nabla} \times\left(\boldsymbol{\zeta} \times \boldsymbol{b}^{* \prime}\right)\right)+\left[\boldsymbol{\zeta}, \boldsymbol{u}^{* \prime}\right] \times \overline{\boldsymbol{b}^{*}}+\boldsymbol{\nabla}\left(\boldsymbol{\zeta} \cdot \boldsymbol{E}^{* \prime}\right) .
$$


Averaging and forming the sum $\overline{\boldsymbol{E}^{*}}=-\overline{\boldsymbol{u}^{*}} \times \overline{\boldsymbol{b}^{*}}-\overline{\boldsymbol{u}^{* \prime} \times \boldsymbol{b}^{* \prime}}$ establishes that the effective electric field $\boldsymbol{E}^{\dagger} \approx \overline{\boldsymbol{E}^{*}}+\frac{1}{2} \partial_{t}\left(\overline{\boldsymbol{\zeta} \times \boldsymbol{b}^{* \prime}}\right)+\frac{1}{2} \boldsymbol{\nabla}\left(\overline{\boldsymbol{\zeta} \cdot \boldsymbol{E}^{* \prime}}\right.$ ) (see (26a)) satisfies $\boldsymbol{E}^{\dagger}=-\boldsymbol{u}^{\dagger} \times \boldsymbol{b}^{\dagger}$ (see (27b)), in which the effective velocity is $\boldsymbol{u}^{\dagger} \approx \overline{\boldsymbol{v}^{*}}+\frac{1}{2} \overline{\left[\boldsymbol{\zeta}, \boldsymbol{v}^{* \prime}\right]}$ and effective magnetic field is $\boldsymbol{b}^{\dagger} \approx \overline{\boldsymbol{b}^{*}}-\frac{1}{2} \boldsymbol{\nabla} \times\left(\overline{\boldsymbol{\zeta} \times \boldsymbol{b}^{* \prime}}\right)$ (see $\left.(25 \mathrm{a}, \mathrm{b})\right)$. It then readily follows from (C.5) that $\partial_{t} \boldsymbol{b}^{\dagger}=-\boldsymbol{\nabla} \times \boldsymbol{E}^{\dagger}$ (see $\left(27\right.$ a) ) holds, when $\partial_{t} \overline{\boldsymbol{b}^{*}}=-\boldsymbol{\nabla} \times \overline{\boldsymbol{E}^{*}}$.

- Effective density, $\rho^{\dagger}$. Our argument parallels that above. Use of $\rho^{* \prime} \simeq-\nabla \cdot\left(\overline{\rho^{*}} \boldsymbol{\zeta}\right)$ leads to

$$
2 \rho^{* \prime} \boldsymbol{u}^{* \prime}-\rho^{* \prime} \partial_{t} \boldsymbol{\zeta} \approx-\rho^{* \prime}\left[\boldsymbol{\zeta}, \overline{\boldsymbol{u}^{*}}\right]-\left(\boldsymbol{\nabla} \cdot\left(\overline{\rho^{*}} \boldsymbol{\zeta}\right)\right) \boldsymbol{u}^{* \prime} .
$$

With the help of (B.7a) the right-hand side becomes

$$
\begin{aligned}
& =\overline{\boldsymbol{u}^{*}} \boldsymbol{\nabla} \cdot\left(\rho^{* \prime} \boldsymbol{\zeta}\right)-\llbracket\left[\boldsymbol{\zeta},\left(\rho^{* \prime} \overline{\boldsymbol{u}^{*}}\right)\right]+\overline{\rho^{*}}\left[\boldsymbol{\zeta}, \boldsymbol{u}^{* \prime}\right]-\llbracket\left[\boldsymbol{\zeta},\left(\overline{\rho^{*}} \boldsymbol{u}^{* \prime}\right) \rrbracket\right. \\
& \left.\approx \overline{\boldsymbol{u}^{*}} \boldsymbol{\nabla} \cdot\left(\rho^{* \prime} \boldsymbol{\zeta}\right)+\overline{\rho^{*}}\left[\boldsymbol{\zeta}, \boldsymbol{u}^{* \prime}\right]-\llbracket \boldsymbol{\zeta}, \boldsymbol{m}^{* \prime}\right],
\end{aligned}
$$

since $\boldsymbol{m}^{* \prime} \simeq \rho^{* \prime} \overline{\boldsymbol{u}^{*}}+\overline{\rho^{*}} \boldsymbol{u}^{* \prime}$. Noting the identity $\llbracket \boldsymbol{\zeta}, \boldsymbol{m}^{* \prime} \rrbracket=\boldsymbol{\zeta} \boldsymbol{\nabla} \cdot \boldsymbol{m}^{* \prime}-\boldsymbol{\nabla} \times\left(\boldsymbol{\zeta} \times \boldsymbol{m}^{* \prime}\right)$ (see $(10 \mathrm{~b})$ ) and use of $\partial_{t} \rho^{* \prime}=-\boldsymbol{\nabla} \cdot \boldsymbol{m}^{* \prime}$ enables us to reduce (C.6a,c) to

$$
2 \rho^{* \prime} \boldsymbol{u}^{* \prime} \approx \partial_{t}\left(\boldsymbol{\zeta} \rho^{* \prime}\right)+\overline{\boldsymbol{u}^{*}} \boldsymbol{\nabla} \cdot\left(\rho^{* \prime} \boldsymbol{\zeta}\right)+\overline{\rho^{*}}\left[\boldsymbol{\zeta}, \boldsymbol{u}^{* \prime}\right]+\boldsymbol{\nabla} \times\left(\boldsymbol{\zeta} \times \boldsymbol{m}^{* \prime}\right) .
$$

Averaging and forming the sum $\overline{\boldsymbol{m}^{*}}=\overline{\rho^{*}} \overline{\boldsymbol{u}^{*}}+\overline{\rho^{* \prime} \boldsymbol{u}^{* \prime}}$ establishes that the effective mass flux $\boldsymbol{m}^{\dagger} \approx \overline{\boldsymbol{m}^{*}}-\frac{1}{2} \partial_{t}\left(\overline{\rho^{* \prime} \boldsymbol{\zeta}}\right)-\frac{1}{2} \boldsymbol{\nabla} \times\left(\overline{\boldsymbol{\zeta} \times \boldsymbol{m}^{* \prime}}\right)($ see $(26 \mathrm{~b}))$ satisfies $\boldsymbol{m}^{\dagger}=\rho^{\dagger} \boldsymbol{u}^{\dagger}$ (see $(28 \mathrm{~b})$ ), in which the effective density is $\rho^{\dagger} \approx \overline{\rho^{*}}+\frac{1}{2} \nabla \cdot\left(\overline{\rho^{* \prime} \zeta}\right.$ ) (see (25c)). It then readily follows from (C.7) that $\partial_{t} \rho^{\dagger}=-\boldsymbol{\nabla} \cdot \boldsymbol{m}^{\dagger}$ (see $\left.(28 \mathrm{a})\right)$ holds, when $\partial_{t} \overline{\rho^{*}}=-\boldsymbol{\nabla} \cdot \overline{\boldsymbol{m}^{*}}$.

- Effective temperature, $\theta^{\dagger}$. Proceeding as above, use of $\theta^{* \prime} \simeq-\boldsymbol{\zeta} \cdot \nabla \overline{\theta^{*}}$ leads to

$$
2 \boldsymbol{u}^{* \prime} \cdot \nabla \theta^{* \prime}-\left(\partial_{t} \boldsymbol{\zeta}\right) \cdot \nabla \theta^{* \prime} \approx-\left[\boldsymbol{\zeta}, \overline{\boldsymbol{u}^{*}}\right] \cdot \nabla \theta^{* \prime}-\boldsymbol{u}^{* \prime} \cdot \nabla\left(\boldsymbol{\zeta} \cdot \nabla \overline{\theta^{*}}\right) .
$$

With the help of (B.8) the right-had side becomes

$$
=\overline{\boldsymbol{u}^{*}} \cdot \boldsymbol{\nabla}\left(\boldsymbol{\zeta} \cdot \boldsymbol{\nabla} \theta^{* \prime}\right)-\boldsymbol{\zeta} \cdot \boldsymbol{\nabla}\left(\overline{\boldsymbol{u}^{*}} \cdot \boldsymbol{\nabla} \theta^{* \prime}\right)+\left[\boldsymbol{\zeta}, \boldsymbol{u}^{* \prime}\right] \cdot \nabla \overline{\theta^{*}}-\boldsymbol{\zeta} \cdot \boldsymbol{\nabla}\left(\boldsymbol{u}^{* \prime} \cdot \boldsymbol{\nabla} \overline{\theta^{*}}\right) .
$$

On use of $\partial_{t} \theta^{* \prime} \simeq-\overline{\boldsymbol{u}^{*}} \cdot \nabla \theta^{* \prime}-\boldsymbol{u}^{* \prime} \cdot \nabla \overline{\theta^{*}},(\mathrm{C} .8 \mathrm{a}, \mathrm{b})$ reduces to

$$
2 \boldsymbol{u}^{* \prime} \cdot \nabla \theta^{* \prime} \approx \partial_{t}\left(\boldsymbol{\zeta} \cdot \nabla \theta^{* \prime}\right)+\overline{\boldsymbol{u}^{*}} \cdot \boldsymbol{\nabla}\left(\boldsymbol{\zeta} \cdot \boldsymbol{\nabla} \theta^{* \prime}\right)+\left[\boldsymbol{\zeta}, \boldsymbol{u}^{* \prime}\right] \cdot \nabla \overline{\theta^{*}} .
$$

Averaging and forming the sum $\overline{\boldsymbol{u}^{*} \cdot \nabla} \overline{\theta^{*}}+\overline{\boldsymbol{u}^{* \prime} \cdot \boldsymbol{\nabla} \theta^{* \prime}}$ shows that the effective temperature $\theta^{\dagger}=\overline{\theta^{*}}+\frac{1}{2} \overline{\boldsymbol{\zeta} \cdot \boldsymbol{\nabla} \theta^{* \prime}}\left(\right.$ see $(25 \mathrm{~d})$ ) satisfies $\partial_{t} \theta^{\dagger}+\boldsymbol{u}^{\dagger} \cdot \boldsymbol{\nabla} \theta^{\dagger}=0$ (see (29)), when

$\partial_{t} \overline{\theta^{*}}=-\overline{\boldsymbol{u}^{*}} \cdot \nabla \overline{\theta^{*}}-\overline{\boldsymbol{u}^{* \prime} \cdot \nabla \theta^{* \prime}}$.

\section{Appendix D: The consequences of a small diffusivity}

In Appendices D.1 and D.2 that follow, we modify the arguments of Appendix C leading to the formulae for fluctuating and mean quantities, reported in section 1.3 for the case of small magnetic and thermal diffusion, $\mathfrak{K}$ and $\kappa$ (also $q^{*} \neq 0$ ), under the assumption that Reynolds and Péclet numbers are large $(R m \gg 1$ and $P e \gg 1$, see $(8 \mathrm{a}, \mathrm{b}))$.

\section{D.1 The equations governing $b^{\dagger \prime}$ and $\theta^{\dagger \prime}$ at $\mathrm{O}(\zeta)$}

Whereas in Appendix C. 1 the effective-variables $\boldsymbol{b}^{\dagger}$ and $\theta^{\dagger}$ were mean quantities, with diffusion included they have fluctuating parts, the equations for which we derive below. 
- The fluctuating effective magnetic field, $\boldsymbol{b}^{\dagger \prime}$. As in the perfectly conducting case, we begin by considering an identity (C.1a) for the expression $\overline{\boldsymbol{u}^{*}} \times\left(\boldsymbol{\nabla} \times\left(\boldsymbol{\zeta} \times \overline{\boldsymbol{b}^{*}}\right)\right)+\boldsymbol{u}^{* \prime} \times \overline{\boldsymbol{b}^{*}}$. In it we substitute $\boldsymbol{\nabla} \times\left(\boldsymbol{\zeta} \times \overline{\boldsymbol{b}^{*}}\right) \bumpeq \boldsymbol{b}^{* \prime}-\boldsymbol{b}^{\dagger \prime}$ (see (33a)) and utilise $\partial_{t} \overline{\boldsymbol{b}^{*}}=-\boldsymbol{\nabla} \times \overline{\boldsymbol{E}^{*}}$, where

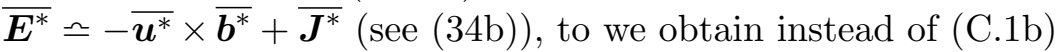

$$
\overline{\boldsymbol{u}^{*}} \times\left(\boldsymbol{b}^{* \prime}-\boldsymbol{b}^{\dagger \prime}\right)+\boldsymbol{u}^{* \prime} \times \overline{\boldsymbol{b}^{*}} \bumpeq \partial_{t}\left(\boldsymbol{\zeta} \times \overline{\boldsymbol{b}^{*}}\right)+\nabla\left(\boldsymbol{\zeta} \cdot \overline{\boldsymbol{E}^{*}}\right)-\left\{\boldsymbol{\zeta}, \overline{\boldsymbol{J}^{*}}\right\},
$$

where, of course, $\left\{\boldsymbol{\zeta}, \overline{\boldsymbol{J}^{*}}\right\}=-\boldsymbol{\zeta} \times\left(\boldsymbol{\nabla} \times \overline{\boldsymbol{J}^{*}}\right)+\boldsymbol{\nabla}\left(\boldsymbol{\zeta} \cdot \overline{\boldsymbol{J}^{*}}\right)$. On the one hand, if we make the postulate $\partial_{t}\left(\boldsymbol{\zeta} \times \overline{\boldsymbol{b}^{*}}\right)+\boldsymbol{\nabla}\left(\boldsymbol{\zeta} \cdot \overline{\boldsymbol{E}^{*}}\right) \bumpeq \boldsymbol{E}^{\dagger \prime}-\boldsymbol{E}^{* \prime}$ (see (34a)) and recall that the fluctuating electric field is $\boldsymbol{E}^{* \prime} \bumpeq-\overline{\boldsymbol{u}^{*}} \times \boldsymbol{b}^{* \prime}-\boldsymbol{u}^{* \prime} \times \overline{\boldsymbol{b}^{*}}+\boldsymbol{J}^{* \prime}$ (see (31a)), (D.1) yields the relation

$$
\boldsymbol{E}^{\dagger \prime} \bumpeq-\overline{\boldsymbol{u}^{*}} \times \boldsymbol{b}^{\dagger \prime}+\left(\boldsymbol{J}^{* \prime}+\left\{\boldsymbol{\zeta}, \overline{\boldsymbol{J}^{*}}\right\}\right)
$$

(see (35b)) or, on adding $\boldsymbol{E}^{* \prime}$,

$$
\widetilde{\boldsymbol{E}}^{\dagger \prime} \bumpeq-\overline{\boldsymbol{u}^{*}} \times \widetilde{\boldsymbol{b}}^{\dagger \prime}-\boldsymbol{u}^{* \prime} \times \overline{\boldsymbol{b}^{*}}+\left(2 \boldsymbol{J}^{* \prime}+\left\{\boldsymbol{\zeta}, \overline{\boldsymbol{J}^{*}}\right\}\right),
$$

where $\widetilde{\boldsymbol{b}}^{\dagger \prime} \equiv \boldsymbol{b}^{\dagger \prime}+\boldsymbol{b}^{* \prime}$ and $\widetilde{\boldsymbol{E}}^{\dagger \prime} \equiv \boldsymbol{E}^{\dagger \prime}+\boldsymbol{E}^{* \prime}$ (see (38a,b)). On the other hand, the curl of (D.1) determines

$$
\partial_{t}\left(\boldsymbol{b}^{* \prime}-\boldsymbol{b}^{\dagger \prime}\right) \bumpeq \nabla \times\left(\overline{\boldsymbol{u}^{*}} \times\left(\boldsymbol{b}^{* \prime}-\boldsymbol{b}^{\dagger \prime}\right)+\boldsymbol{u}^{* \prime} \times \overline{\boldsymbol{b}^{*}}+\left\{\boldsymbol{\zeta}, \overline{\boldsymbol{J}^{*}}\right\}\right),
$$

which on forming the difference with $\partial_{t} \boldsymbol{b}^{* \prime}=-\boldsymbol{\nabla} \times \boldsymbol{E}^{* \prime} \bumpeq \boldsymbol{\nabla} \times\left(\overline{\boldsymbol{u}^{*}} \times \boldsymbol{b}^{* \prime}+\boldsymbol{u}^{* \prime} \times \overline{\boldsymbol{b}^{*}}-\boldsymbol{J}^{* \prime}\right)$ determines

$$
\partial_{t} \boldsymbol{b}^{\dagger \prime} \bumpeq \nabla \times\left(\overline{\boldsymbol{u}^{*}} \times \boldsymbol{b}^{\dagger \prime}-\left(\boldsymbol{J}^{* \prime}+\left\{\boldsymbol{\zeta}, \overline{\boldsymbol{J}^{*}}\right\}\right)\right),
$$

which establishes $\partial_{t} \boldsymbol{b}^{\dagger \prime}=-\boldsymbol{\nabla} \times \boldsymbol{E}^{\dagger \prime}$ (see (35a)) or equivalently

$$
\partial_{t} \widetilde{\boldsymbol{b}}^{\dagger \prime}=-\nabla \times \widetilde{\boldsymbol{E}}^{\dagger \prime} .
$$

- The fluctuating effective temperature, $\theta^{\dagger \prime}$. As in the insulating case, we begin with the identity (C.3a) for the expression $-\overline{\boldsymbol{u}^{*}} \cdot \boldsymbol{\nabla}\left(\boldsymbol{\zeta} \cdot \boldsymbol{\nabla} \overline{\theta^{*}}\right)+\boldsymbol{u}^{* \prime} \cdot \boldsymbol{\nabla} \overline{\theta^{*}}$. In it we substitute $-\boldsymbol{\zeta} \cdot \boldsymbol{\nabla} \overline{\theta^{*}} \bumpeq$ $\theta^{* \prime}-\theta^{\dagger \prime}($ see $(33 \mathrm{~b}))$ and utilise $\partial_{t} \overline{\theta^{*}}+\overline{u^{*}} \cdot \nabla \overline{\theta^{*}} \bumpeq \kappa \Delta \overline{\theta^{*}}+\overline{q^{*}}$ (see $(7 \mathrm{a}, \mathrm{b})$ ) to obtain instead of (C.3b)

$$
\partial_{t}\left(\theta^{* \prime}-\theta^{\dagger \prime}\right)+\overline{\boldsymbol{u}^{*}} \cdot \nabla\left(\theta^{* \prime}-\theta^{\dagger \prime}\right)+\boldsymbol{u}^{* \prime} \cdot \nabla \overline{\theta^{*}} \bumpeq-\kappa \boldsymbol{\zeta} \cdot \nabla\left(\Delta \overline{\theta^{*}}\right)-\boldsymbol{\zeta} \cdot \nabla \overline{q^{*}} .
$$

Then on forming the difference of the result (D.4) and the fluctuating heat conduction equation $\partial_{t} \theta^{* \prime}+\overline{u^{*}} \cdot \nabla \theta^{* \prime}+\boldsymbol{u}^{* \prime} \cdot \nabla \overline{\theta^{*}} \bumpeq \kappa \Delta \theta^{* \prime}+q^{* \prime}$ (see $(7 \mathrm{c})$ ), we obtain

$$
\partial_{t} \theta^{\dagger \prime}+\overline{\boldsymbol{u}^{*}} \cdot \nabla \theta^{\dagger \prime} \bumpeq \kappa\left(\Delta \theta^{* \prime}+\boldsymbol{\zeta} \cdot \nabla\left(\Delta \overline{\theta^{*}}\right)\right)+q^{\dagger \prime},
$$

where $q^{\dagger \prime} \bumpeq q^{* \prime}+\boldsymbol{\zeta} \cdot \nabla \overline{q^{*}}$ (see (36b)) or equivalently

$$
\partial_{t} \widetilde{\theta}^{\dagger \prime}+\overline{\boldsymbol{u}^{*}} \cdot \boldsymbol{\nabla} \widetilde{\theta}^{\dagger \prime}+\boldsymbol{u}^{* \prime} \cdot \nabla \overline{\theta^{*}} \bumpeq \kappa\left(2 \Delta \theta^{* \prime}+\boldsymbol{\zeta} \cdot \boldsymbol{\nabla}\left(\Delta \overline{\theta^{*}}\right)\right)+\widetilde{q}^{\dagger \prime},
$$

where $\widetilde{\theta}^{\dagger \prime}=\theta^{* \prime}+\theta^{\dagger \prime}, \quad \widetilde{q}^{\dagger \prime} \bumpeq q^{* \prime}+q^{\dagger \prime}($ see $(39 \mathrm{c})$ and $(40 \mathrm{e}))$.

\section{D.2 The equations governing $\overline{b^{\dagger}}$ and $\overline{\theta^{\dagger}}$ at $\mathrm{O}\left(\zeta^{2}\right)$}

Armed with the equations for the fluctuating variables $\boldsymbol{b}^{\dagger \prime}$ and $\theta^{\dagger \prime}$ at $\mathrm{O}(\zeta)$ obtained in Appendix D.1, we proceed with our main objective to obtain the equations governing the mean effective-variables $\overline{\boldsymbol{b}^{\dagger}}$ and $\overline{\theta^{\dagger}}$ correct to $\mathrm{O}\left(\zeta^{2}\right)$. 
- The mean effective magnetic field, $\overline{\boldsymbol{b}^{\dagger}}$. As a preliminary step we add $\boldsymbol{u}^{* \prime} \times\left(\boldsymbol{b}^{* \prime}-\boldsymbol{b}^{\dagger \prime}\right) \approx$ $\boldsymbol{u}^{* \prime} \times\left(\boldsymbol{\nabla} \times\left(\boldsymbol{\zeta} \times \overline{\boldsymbol{b}^{*}}\right)\right)$ to the identity $\boldsymbol{u}^{* \prime} \times \widetilde{\boldsymbol{b}}^{\dagger \prime}-\left(\partial_{t} \boldsymbol{\zeta}\right) \times \widetilde{\boldsymbol{b}}^{\dagger \prime}=-\left[\boldsymbol{\zeta}, \overline{\boldsymbol{u}^{*}}\right] \times \widetilde{\boldsymbol{b}}^{\dagger \prime}$ (remember that $\left.\widetilde{\boldsymbol{b}}^{\dagger \prime}=\boldsymbol{b}^{* \prime}+\boldsymbol{b}^{\dagger \prime}\right)$ to obtain

$$
2 \boldsymbol{u}^{* \prime} \times \boldsymbol{b}^{* \prime}-\left(\partial_{t} \boldsymbol{\zeta}\right) \times \widetilde{\boldsymbol{b}}^{\dagger \prime} \approx-\left[\boldsymbol{\zeta}, \overline{\boldsymbol{u}^{*}}\right] \times \widetilde{\boldsymbol{b}}^{\dagger \prime}+\boldsymbol{u}^{* \prime} \times\left(\boldsymbol{\nabla} \times\left(\boldsymbol{\zeta} \times \overline{\boldsymbol{b}^{*}}\right)\right)
$$

identical to (C.4a) but with the $\boldsymbol{b}^{* \prime}$ replaced by $\widetilde{\boldsymbol{b}}^{\dagger \prime}$ in the terms adjacent to the $\approx$ sign. The corresponding form to (C.4b) follows trivially, while with the help of the expression (D.2b) for $\widetilde{\boldsymbol{E}}^{\dagger \prime}$ the right-hand side of (C.4c) takes on the modified form

$$
\approx-\overline{\boldsymbol{u}^{*}} \times\left(\boldsymbol{\nabla} \times\left(\boldsymbol{\zeta} \times \widetilde{\boldsymbol{b}}^{\dagger \prime}\right)\right)+\left[\boldsymbol{\zeta}, \boldsymbol{u}^{* \prime}\right] \times \overline{\boldsymbol{b}^{*}}+\left\{\boldsymbol{\zeta}, \widetilde{\boldsymbol{E}}^{\dagger \prime}\right\}-\left\{\boldsymbol{\zeta},\left(2 \boldsymbol{J}^{* \prime}+\left\{\boldsymbol{\zeta}, \overline{\boldsymbol{J}^{*}}\right\}\right)\right\} .
$$

On use of $\partial_{t} \widetilde{\boldsymbol{b}}^{\dagger \prime}=-\boldsymbol{\nabla} \times \widetilde{\boldsymbol{E}}^{\dagger \prime}$ (see (D.3c)), the diffusive result corresponding to (C.5) is

$$
\begin{aligned}
2 \boldsymbol{u}^{* \prime} \times \boldsymbol{b}^{* \prime}-\partial_{t}\left(\boldsymbol{\zeta} \times \widetilde{\boldsymbol{b}}^{\dagger \prime}\right)+\overline{\boldsymbol{u}^{*}} \times\left(\boldsymbol{\nabla} \times\left(\boldsymbol{\zeta} \times \widetilde{\boldsymbol{b}}^{\dagger \prime}\right)\right)-\left[\boldsymbol{\zeta}, \boldsymbol{u}^{* \prime}\right] \times \overline{\boldsymbol{b}^{*}} \\
\approx \boldsymbol{\nabla}\left(\boldsymbol{\zeta} \cdot \widetilde{\boldsymbol{E}}^{\dagger \prime}\right)-\left\{\boldsymbol{\zeta},\left(2 \boldsymbol{J}^{* \prime}+\left\{\boldsymbol{\zeta}, \overline{\boldsymbol{J}^{*}}\right\}\right)\right\} .
\end{aligned}
$$

On the one hand, making the postulate $\overline{\boldsymbol{E}^{\dagger}} \approx \overline{\boldsymbol{E}^{*}}+\frac{1}{2} \partial_{t}\left(\overline{\boldsymbol{\zeta} \times \widetilde{\boldsymbol{b}}^{\dagger \prime}}\right)+\frac{1}{2} \boldsymbol{\nabla}\left(\overline{\boldsymbol{\zeta} \cdot \widetilde{\boldsymbol{E}}^{\dagger \prime}}\right)$ (see (40b)), in which $\overline{\boldsymbol{E}^{*}}=-\overline{\boldsymbol{u}^{*}} \times \overline{\boldsymbol{b}^{*}}-\overline{\boldsymbol{u}^{* \prime} \times \boldsymbol{b}^{* \prime}}+\overline{\boldsymbol{J}^{*}}$, it follows from the mean of (D.7) that

$$
\overline{\boldsymbol{E}^{\dagger}} \approx-\boldsymbol{u}^{\dagger} \times \overline{\boldsymbol{b}^{\dagger}}+\left(\overline{\boldsymbol{J}^{*}}+\overline{\left\{\boldsymbol{\zeta}, \boldsymbol{J}^{* \prime}\right\}}+\frac{1}{2} \overline{\left\{\boldsymbol{\zeta},\left\{\boldsymbol{\zeta}, \overline{\boldsymbol{J}^{*}}\right\}\right\}}\right)
$$

(see (41b)), where $\overline{\boldsymbol{b}^{\dagger}} \approx \overline{\boldsymbol{b}^{*}}-\frac{1}{2} \boldsymbol{\nabla} \times\left(\overline{\boldsymbol{\zeta} \times \widetilde{\boldsymbol{b}}^{\dagger \prime}}\right.$ ) (see (40a)). On the other hand, the curl of (D.7) averaged simply establishes that $\overline{\boldsymbol{E}^{\dagger}}$ defined by (D.8a) satisfies

$$
\partial_{t} \overline{\boldsymbol{b}^{\dagger}}=-\nabla \times \overline{\boldsymbol{E}^{\dagger}}
$$

(see (41a)).

- The mean effective temperature, $\overline{\theta^{\dagger}}$. As a preliminary step we add $\boldsymbol{u}^{* \prime} \cdot \boldsymbol{\nabla}\left(\theta^{* \prime}-\theta^{\dagger \prime}\right) \approx$ $-\boldsymbol{u}^{* \prime} \cdot \boldsymbol{\nabla}\left(\boldsymbol{\zeta} \cdot \boldsymbol{\nabla} \overline{\theta^{*}}\right)$ to the identity $\boldsymbol{u}^{* \prime} \cdot \boldsymbol{\nabla} \widetilde{\theta}^{\dagger \prime}-\left(\partial_{t} \boldsymbol{\zeta}\right) \cdot \boldsymbol{\nabla} \widetilde{\theta}^{\dagger \prime}=-\left[\boldsymbol{\zeta}, \overline{\boldsymbol{u}^{*}}\right] \cdot \boldsymbol{\nabla} \widetilde{\theta}^{\dagger \prime}$ (remember that $\left.\tilde{\theta}^{\dagger \prime}=\theta^{* \prime}+\theta^{\dagger \prime}\right)$ to obtain

$$
2 \boldsymbol{u}^{* \prime} \cdot \nabla \theta^{* \prime}-\left(\partial_{t} \boldsymbol{\zeta}\right) \cdot \nabla \widetilde{\theta}^{\dagger \prime} \approx-\left[\boldsymbol{\zeta}, \overline{\boldsymbol{u}^{*}}\right] \cdot \nabla \widetilde{\theta}^{\dagger \prime}-\boldsymbol{u}^{* \prime} \cdot \nabla\left(\boldsymbol{\zeta} \cdot \nabla \overline{\theta^{*}}\right)
$$

identical to (C.8a) but with the $\theta^{* \prime}$ replaced by $\widetilde{\theta}^{\dagger \prime}$ in the terms adjacent to the $\approx$ sign. The corresponding form to (C.8b) follows trivially. Then on use of the value of $\partial_{t} \widetilde{\theta}^{\dagger \prime}$ given by (D.5b), the result corresponding to (C.9) is

$$
\begin{aligned}
2 \boldsymbol{u}^{* \prime} \cdot \boldsymbol{\nabla} \theta^{* \prime} \approx & \partial_{t}\left(\boldsymbol{\zeta} \cdot \boldsymbol{\nabla} \widetilde{\theta}^{\dagger \prime}\right)+\overline{\boldsymbol{u}^{*}} \cdot \boldsymbol{\nabla}\left(\boldsymbol{\zeta} \cdot \boldsymbol{\nabla} \widetilde{\theta}^{\dagger \prime}\right)+\left[\boldsymbol{\zeta}, \boldsymbol{u}^{* \prime}\right] \cdot \boldsymbol{\nabla} \overline{\theta^{*}} \\
& -\kappa \boldsymbol{\zeta} \cdot \boldsymbol{\nabla}\left(2 \Delta \theta^{* \prime}+\boldsymbol{\zeta} \cdot \boldsymbol{\nabla}\left(\Delta \overline{\theta^{*}}\right)\right)-\boldsymbol{\zeta} \cdot \boldsymbol{\nabla} \widetilde{q}^{\dagger \prime} .
\end{aligned}
$$

The substitution of the average into the mean equation $\partial_{t} \overline{\theta^{*}}+\overline{\boldsymbol{u}^{*}} \cdot \boldsymbol{\nabla} \overline{\theta^{*}}+\overline{\boldsymbol{u}^{* \prime} \cdot \boldsymbol{\nabla} \theta^{* \prime}}=\kappa \Delta \overline{\theta^{*}}+\overline{q^{*}}$ leads directly to the effective mean heat conduction equation

$$
\left.\partial_{t} \overline{\theta^{\dagger}}+\boldsymbol{u}^{\dagger} \cdot \boldsymbol{\nabla} \overline{\theta^{\dagger}} \approx \kappa\left(\Delta \overline{\theta^{*}}+\overline{\boldsymbol{\zeta} \cdot \boldsymbol{\nabla}\left(\Delta \theta^{* \prime}+\frac{1}{2} \boldsymbol{\zeta} \cdot \boldsymbol{\nabla}\left(\Delta \overline{\theta^{*}}\right)\right.}\right)\right)+\overline{q^{\dagger}}
$$

(see (42)), where $\overline{\theta^{\dagger}} \approx \overline{\theta^{*}}+\frac{1}{2} \overline{\zeta \cdot \nabla \widetilde{\theta}^{\dagger \prime}}, \overline{q^{\dagger}} \approx \overline{q^{*}}+\frac{1}{2} \overline{\zeta \cdot \nabla \widetilde{q}^{\dagger \prime}}$ (see (40c,d)). 


\section{Appendix E: The Coriolis acceleration}

We introduce the notation

$$
\mathcal{X}=2 \boldsymbol{x}+\boldsymbol{\xi} \quad \Rightarrow \quad 2 \mathbf{I}=\boldsymbol{\nabla} \mathcal{X}-\nabla \boldsymbol{\xi}, \quad \mathcal{V}=\boldsymbol{\Omega} \times \boldsymbol{\xi}
$$

with the immediate consequences

$$
\begin{gathered}
(\boldsymbol{\nabla} \mathcal{V}) \cdot \boldsymbol{u}=-(\boldsymbol{\nabla}) \cdot(\boldsymbol{\Omega} \times \boldsymbol{u})=-\left(\boldsymbol{\nabla} \boldsymbol{x}^{L}\right) \cdot(\boldsymbol{\Omega} \times \boldsymbol{u})+\boldsymbol{\Omega} \times \boldsymbol{u}, \\
(\boldsymbol{\nabla} \mathcal{V}) \cdot\left(\mathrm{D}_{t} \boldsymbol{\xi}\right)=-(\boldsymbol{\nabla} \boldsymbol{\xi}) \cdot\left(\mathrm{D}_{t} \mathcal{V}\right)=-\left(\boldsymbol{\nabla} \boldsymbol{x}^{L}\right) \cdot\left(\boldsymbol{\Omega} \times \mathrm{D}_{t} \boldsymbol{\xi}\right)+\boldsymbol{\Omega} \times \mathrm{D}_{t} \boldsymbol{\xi} .
\end{gathered}
$$

Recalling that $\boldsymbol{u}^{* L}=\boldsymbol{u}+\mathrm{D}_{t} \boldsymbol{\xi}$ (see (66a)), our objective is the reduction of

$$
\begin{aligned}
\left(\boldsymbol{\nabla} \boldsymbol{x}^{L}\right) \cdot \mathcal{C}^{* L} & =\left(\boldsymbol{\nabla} \boldsymbol{x}^{L}\right) \cdot\left(2 \boldsymbol{\Omega} \times \boldsymbol{u}^{* L}\right)=\left(\boldsymbol{\nabla} \boldsymbol{x}^{L}\right) \cdot\left(2 \boldsymbol{\Omega} \times\left(\boldsymbol{u}+\mathrm{D}_{t} \boldsymbol{\xi}\right)\right) \\
& =2 \boldsymbol{\Omega} \times\left(\boldsymbol{u}+\mathrm{D}_{t} \boldsymbol{\xi}\right)-2(\boldsymbol{\nabla} \boldsymbol{V}) \cdot\left(\boldsymbol{u}+\mathrm{D}_{t} \boldsymbol{\xi}\right),
\end{aligned}
$$

on use of (E.2a,b). Further manipulations yield

$$
\begin{aligned}
\left(\boldsymbol{\nabla} \boldsymbol{x}^{L}\right) \cdot \mathcal{C}^{* L}-2 \boldsymbol{\Omega} \times \boldsymbol{u} & =2 \boldsymbol{I} \cdot\left(\mathrm{D}_{t} \mathcal{V}\right)-2(\boldsymbol{\nabla} \mathcal{V}) \cdot\left(\boldsymbol{u}+\mathrm{D}_{t} \boldsymbol{\xi}\right) \\
& =(\boldsymbol{\nabla} \mathcal{X}) \cdot\left(\mathrm{D}_{t} \mathcal{V}\right)-(\boldsymbol{\nabla} \boldsymbol{\xi}) \cdot\left(\mathrm{D}_{t} \mathcal{V}\right)-2(\boldsymbol{\nabla} \mathcal{V}) \cdot\left(\boldsymbol{u}+\mathrm{D}_{t} \boldsymbol{\xi}\right) \\
& =(\boldsymbol{\nabla} \mathcal{X}) \cdot\left(\mathrm{D}_{t} \mathcal{V}\right)+(\boldsymbol{\nabla} \mathcal{V}) \cdot\left(\mathrm{D}_{t} \boldsymbol{\xi}-2\left(\boldsymbol{u}+\mathrm{D}_{t} \boldsymbol{\xi}\right)\right) \\
& =(\boldsymbol{\nabla} \mathcal{X}) \cdot\left(\mathrm{D}_{t} \mathcal{V}\right)-(\boldsymbol{\nabla} \mathcal{V}) \cdot\left(\mathrm{D}_{t} \boldsymbol{\mathcal { X }}\right)
\end{aligned}
$$

on sequential use in each line of (E.1c), (E.1b), (E.2b) and (E.1a).

We introduce the representation $\mathcal{R}=(\boldsymbol{\nabla} \mathcal{X}) \cdot \mathcal{V}$ (see the near right-hand side of (131)):

$$
\mathcal{R}=\left(\mathbf{I}+\boldsymbol{\nabla} \boldsymbol{x}^{L}\right) \cdot \mathcal{V}=2 \mathcal{V}+(\boldsymbol{\nabla} \boldsymbol{\xi}) \cdot \mathcal{V}=(\boldsymbol{\nabla} \mathcal{X}) \cdot \mathcal{V}
$$

with the property

$$
\begin{aligned}
(\boldsymbol{\nabla} \mathcal{R}) \cdot \boldsymbol{u} & =(\boldsymbol{\nabla}((\boldsymbol{\nabla} \mathcal{X}) \cdot \mathcal{V})) \cdot \boldsymbol{u} \\
& =((\boldsymbol{u} \cdot \boldsymbol{\nabla})(\boldsymbol{\nabla} \mathcal{X})) \cdot \mathcal{V}+(\boldsymbol{\nabla} \mathcal{V}) \cdot((\boldsymbol{u} \cdot \boldsymbol{\nabla}) \mathcal{X}) \\
& =\left(\mathrm{D}_{t}(\boldsymbol{\nabla} \mathcal{X})\right) \cdot \mathcal{V}+(\boldsymbol{\nabla} \mathcal{V}) \cdot\left(\mathrm{D}_{t} \mathcal{X}\right)-\boldsymbol{\nabla}\left(\mathcal{V} \cdot \partial_{t} \mathcal{X}\right)
\end{aligned}
$$

since $\boldsymbol{u} \cdot \boldsymbol{\nabla}=\mathrm{D}_{t}-\partial_{t}$. Hence we may establish the identity

$$
\begin{aligned}
\partial_{t} \boldsymbol{\mathcal { R }}+(\boldsymbol{\nabla} \times \boldsymbol{\mathcal { R }}) \times \boldsymbol{u} & =\mathrm{D}_{t} \boldsymbol{\mathcal { R }}-(\boldsymbol{\nabla} \mathcal{R}) \cdot \boldsymbol{u} \\
& =(\boldsymbol{\nabla} \mathcal{X}) \cdot\left(\mathrm{D}_{t} \mathcal{V}\right)-(\boldsymbol{\nabla} \mathcal{V}) \cdot\left(\mathrm{D}_{t} \mathcal{X}\right)+\boldsymbol{\nabla}\left(\boldsymbol{V} \cdot \partial_{t} \mathcal{X}\right),
\end{aligned}
$$

on $\mathrm{D}_{t}$-differentiation of (E.4) and use of (E.5). Together (E.3b) and (E.6) determine (132):

$$
\left(\boldsymbol{\nabla} \boldsymbol{x}^{L}\right) \cdot \mathcal{C}^{* L}=2 \boldsymbol{\Omega} \times \boldsymbol{u}+\partial_{t} \boldsymbol{\mathcal { R }}+(\boldsymbol{\nabla} \times \boldsymbol{\mathcal { R }}) \times \boldsymbol{u}-\boldsymbol{\nabla}\left(\mathcal{V} \cdot \partial_{t} \boldsymbol{\xi}\right) .
$$

The application of the useful identity $\delta_{i j} \epsilon_{k l m}=\delta_{k j} \epsilon_{i l m}+\delta_{l j} \epsilon_{k i m}+\delta_{m j} \epsilon_{k l i}($ see (B.1c)) to the term $\mathcal{R}_{i}=((\boldsymbol{\nabla} \mathcal{X}) \cdot(\boldsymbol{\Omega} \times \boldsymbol{\xi}))_{i}=\delta_{i j} \epsilon_{k l m}\left(\nabla_{j} \mathcal{X}_{k}\right)\left(\Omega_{l} \xi_{m}\right)$ (see (E.4)) leads to the alternative representation

$$
\mathcal{R}=\boldsymbol{\Omega} \times((\boldsymbol{\nabla} \cdot \mathcal{X}) \boldsymbol{\xi}-\boldsymbol{\xi} \cdot \boldsymbol{\nabla} \mathcal{X})-(\boldsymbol{\Omega} \cdot \boldsymbol{\nabla} \mathcal{X}) \times \boldsymbol{\xi},
$$

which with $\boldsymbol{\nabla} \mathcal{X}=2 \mathbf{I}+\boldsymbol{\nabla} \boldsymbol{\xi}$ determines the far right-hand side of (131).

\section{Appendix F: The cylindrical polar coordinate reduction of $\mathfrak{K}^{-1} J$}

Our objective in this Appendix is to cast the expression $\mathfrak{K}^{-1} \boldsymbol{J}$ defined by (167a-c) in the form $(169 \mathrm{a}, \mathrm{b})$, from which the average values $\mathfrak{K}^{-1} \overline{J_{\phi}}$ and $\mathfrak{K}^{-1}(\boldsymbol{\nabla} \times \overline{\boldsymbol{J}})_{\phi}($ see $(171 \mathrm{a}, \mathrm{b}))$ may be 
readily calculated from the toroidal-poloidal decomposition $\overline{\boldsymbol{b}}=\overline{b_{\phi}} \widehat{\boldsymbol{\phi}}+\boldsymbol{\nabla} \times(a \widehat{\boldsymbol{\phi}})$ of the mean HEL magnetic field (see (154b)). To achieve that objective we consider the partition

$$
\overline{b^{p}}=\left(\delta^{p 2} \delta^{2 q}+\epsilon^{2 p r} \epsilon^{r 2 q}\right) \overline{b^{q}}
$$

of $\overline{\boldsymbol{b}}$, which naturally identifies the two terms $\overline{b^{p}}=\delta^{p 2} \overline{b_{\phi}}+s^{-1} \epsilon^{2 p r} \nabla^{r}(s a)$ (see (170)). The first term on the right-hand of (F.1) is the toroidal part $\overline{b_{\phi}}=\delta^{2 q} \overline{b^{q}}$, while the second term is the poloidal part $(\nabla \times(a \widehat{\phi}))^{p}=\epsilon^{2 p r} \epsilon^{r 2 q} \overline{b^{q}}$.

We substitute the the partition (F.1) into the expression (167a) for $\mathfrak{K}^{-1} \boldsymbol{J}$. We begin with the term $\epsilon^{p q t} g^{q s} \nabla^{s} \overline{b^{t}}$. For the $\delta^{t 2} \delta^{2 r} \overline{b^{r}}$ contribution we have the simple consequence

$$
\epsilon^{p q t} g^{q s} \nabla^{s}\left(\delta^{t 2} \delta^{2 r} \overline{b^{r}}\right)=\epsilon^{p q 2} g^{q s} s^{-1} \nabla^{s}\left(s \overline{b^{2}}\right)-\epsilon^{2 p s} g^{s 1} s^{-1} \overline{b^{2}} .
$$

The corresponding consequence for the $\epsilon^{2 p r} \epsilon^{r 2 q} \overline{b^{q}}$ contribution is

$$
\epsilon^{p q t} g^{q s} \nabla^{s}\left(\epsilon^{2 t r} \epsilon^{r 2 u} \overline{b^{u}}\right)=\epsilon^{p 2 q} \delta^{r s} g^{2 s} \nabla^{r} \overline{b^{q}}+\delta^{p 2} \epsilon^{2 r q} g^{r s} \nabla^{s} \overline{b^{q}},
$$

since $\epsilon^{p q t} \epsilon^{2 t r}=\delta^{p r} \delta^{q 2}-\delta^{p 2} \delta^{q r}$,

$$
=\left(\epsilon^{s 2 q} \delta^{r p}+\epsilon^{p s q} \delta^{r 2}+\epsilon^{p 2 s} \delta^{r q}\right) g^{2 s} \nabla^{r} \overline{b^{q}}+\delta^{p 2} \epsilon^{2 r q} g^{r s} \nabla^{s} \overline{b^{q}},
$$

because of the useful identity (B.1c) expressed in the form $\epsilon^{p w q} \delta^{r s}=\epsilon^{s w q} \delta^{r p}+\epsilon^{p s q} \delta^{r w}+\epsilon^{p w s} \delta^{r q}$ with $w=2$,

$$
=\left(\epsilon^{s 2 q} g^{2 s} \nabla^{p} \overline{b^{q}}-\epsilon^{p 2 s} g^{2 s} s^{-1} \overline{b^{1}}\right)+\delta^{p 2} \epsilon^{2 r q} g^{r s} \nabla^{s} \overline{b^{q}},
$$

since $\nabla^{2} \overline{b^{q}}=0$ and $\nabla \cdot \overline{\boldsymbol{b}}=\nabla^{q} \overline{b^{q}}+s^{-1} \overline{b^{1}}=0$,

$$
\begin{aligned}
= & \nabla^{p}\left(\epsilon^{s 2 q} g^{2 s} \overline{b^{q}}\right)+\delta^{p 2} \epsilon^{2 r q} \nabla^{s}\left(g^{r s} \overline{b^{q}}\right) \\
& -\epsilon^{s 2 q}\left(\nabla^{p} g^{2 s}\right) \overline{b^{q}}+\epsilon^{2 p s} g^{2 s} s^{-1} \overline{b^{1}}-\delta^{p 2} \epsilon^{2 r q}\left(\nabla^{s} g^{r s}\right) \overline{b^{q}} .
\end{aligned}
$$

On combining (F.2) and (F.3a,b) we obtain

$$
\begin{aligned}
\epsilon^{p q t} g^{q s} \nabla^{s} \overline{b^{t}}= & -\nabla^{p}\left(\epsilon^{2 s q} g^{2 s} \overline{b^{q}}\right)+\epsilon^{p q 2} g^{q s} s^{-1} \nabla^{s}\left(s \overline{b^{2}}\right)+\delta^{p 2} \epsilon^{2 r q} \nabla^{s}\left(g^{r s} \overline{b^{q}}\right) \\
& -\epsilon^{s 2 q}\left(\nabla^{p} g^{2 s}\right) \overline{b^{q}}-\epsilon^{p s 2} \epsilon^{3 r q} g^{s r} s^{-1} \overline{b^{q}}-\delta^{p 2} \epsilon^{2 r q}\left(\nabla^{s} g^{r s}\right) \overline{b^{q}}
\end{aligned}
$$

since $\epsilon^{3 r q}=\delta^{r 1} \delta^{q 2}-\delta^{r 2} \delta^{q 1}$.

Substitution of (F.4) into (167a) yields

$$
\begin{aligned}
\mathfrak{K}^{-1} J^{p}= & -\nabla^{p}\left(\epsilon^{2 r q} g^{2 r} \overline{b^{q}}\right)+\epsilon^{p q 2} g^{q s} s^{-1} \nabla^{s}\left(s \overline{b^{2}}\right)+\delta^{p 2} \epsilon^{2 r q} \nabla^{s}\left(g^{r s} \overline{b^{q}}\right) \\
& -\left(\gamma^{p q}+\frac{1}{2} \epsilon^{p q r} s \nabla^{2}\left(s^{-1} g^{2 r}\right)\right) \overline{b^{q}}
\end{aligned}
$$

where

$$
\gamma^{p q}+\frac{1}{2} \epsilon^{p q r} s \nabla^{2}\left(s^{-1} g^{2 r}\right)=\epsilon^{r 2 q} \nabla^{p} g^{2 r}+\epsilon^{p s 2} \epsilon^{3 r q} s^{-1} g^{s r}+\delta^{p 2} \epsilon^{2 r q} \nabla^{s} g^{r s}+s^{-1} \aleph^{p q} .
$$

Here the term $\frac{1}{2} \epsilon^{p q r} s \nabla^{2}\left(s^{-1} g^{2 r}\right)$ has been added to ensure that $\gamma^{p q}\left(=\gamma^{q p}\right)$, like $g^{r s}\left(=g^{s r}\right)$, is symmetric (see (F.6b)). Together with (168c) we deduce that

$$
\begin{aligned}
2 \gamma^{p q}- & s^{-1}\left(\aleph^{p q}+\aleph^{q p}\right) \\
= & -\epsilon^{p q r} s \nabla^{2}\left(s^{-1} g^{2 r}\right)+2 \epsilon^{r 2 q}\left(s \nabla^{p}\left(s^{-1} g^{2 r}\right)+\delta^{p 1} s^{-1} g^{2 r}\right)+2 \epsilon^{p s 2} \epsilon^{3 r q} s^{-1} g^{s r} \\
& +2 \delta^{p 2} \epsilon^{2 r q}\left(s \nabla^{s}\left(s^{-1} g^{r s}\right)+s^{-1} g^{r 1}\right)+\epsilon^{p q r}\left(s \nabla^{s}\left(s^{-1} g^{r s}\right)+2 s^{-1} g^{r 1}\right) \\
= & -\epsilon^{p q r} s \nabla^{2}\left(s^{-1} g^{2 r}\right)+2 \epsilon^{r 2 q} s \nabla^{p}\left(s^{-1} g^{2 r}\right)+2 \delta^{p 2} \epsilon^{2 r q} s \nabla^{s}\left(s^{-1} g^{r s}\right)+\epsilon^{p q r} s \nabla^{s}\left(s^{-1} g^{r s}\right),
\end{aligned}
$$


since $\left(\epsilon^{r 2 q} \delta^{p 1} g^{2 r}+\delta^{p 2} \epsilon^{2 r q} g^{r 1}\right)+\epsilon^{p s 2} \epsilon^{3 r q} g^{s r}=\left(-\epsilon^{q r 2} \epsilon^{3 s p}+\epsilon^{p s 2} \epsilon^{3 r q}\right) g^{s r}=\epsilon^{q r v} \epsilon^{v 1 u} \epsilon^{u s p} g^{s r}$ $=-\epsilon^{p q r} g^{r 1}$,

$$
\begin{aligned}
& =\left(-\epsilon^{p q r} \delta^{s 2} \delta^{t 2}+2 \epsilon^{r 2 q} \delta^{t p} \delta^{s 2}+2 \epsilon^{2 r q} \delta^{p 2} \delta^{t s}+\epsilon^{p q r} \delta^{t s}\right) s \nabla^{t}\left(s^{-1} g^{r s}\right) \\
& =\left(2 \epsilon^{r 2 q} \epsilon^{u p s} \epsilon^{u t 2}+\epsilon^{p q r} \epsilon^{t u 2} \epsilon^{u 2 s}\right) s \nabla^{t}\left(s^{-1} g^{r s}\right) \\
& =\epsilon^{2 u t} \epsilon^{r v q}\left(2 \epsilon^{u p s} \delta^{v 2}-\epsilon^{u 2 s} \delta^{v p}\right) s \nabla^{t}\left(s^{-1} g^{r s}\right) \\
& =\epsilon^{2 u t} \epsilon^{r v q}\left(2 \epsilon^{u p s} \delta^{v 2}-\left(\epsilon^{p 2 s} \delta^{v u}+\epsilon^{u p s} \delta^{v 2}+\epsilon^{u 2 p} \delta^{v s}\right)\right) s \nabla^{t}\left(s^{-1} g^{r s}\right),
\end{aligned}
$$

again because of the identity $\epsilon^{u w s} \delta^{v p}=\epsilon^{p w s} \delta^{v u}+\epsilon^{u p s} \delta^{v w}+\epsilon^{u w p} \delta^{v s}$ with $w=2$,

$$
=\epsilon^{2 u t} \epsilon^{r v q}\left(\epsilon^{u p s} \delta^{v 2}-\epsilon^{p 2 s} \delta^{v u}\right) s \nabla^{t}\left(s^{-1} g^{r s}\right),
$$

since $\epsilon^{s q r} g^{r s}=0$. This determines (169b) and completes our representation (169a) of $\mathfrak{K}^{-1} J^{p}$.

\section{Appendix G: Effective-variables}

Though the effective and HEL EMF's $\mathcal{E}^{\dagger}$ and $\mathcal{E}$, like $\boldsymbol{b}^{\dagger}$ and $\boldsymbol{b}$, are the same, the values of $\aleph^{\ddagger}$ and $\aleph\left(\approx \aleph^{\ddagger}\right)$ in their approximate relations $\mathcal{E}_{\phi}^{\dagger} \doteq \mathfrak{K} s^{-1} \aleph^{\ddagger} \overline{b_{\phi}^{\dagger}}$ (see $\left.(52 \mathrm{~d})\right)$ and $\mathcal{E}_{\phi} \doteq \mathfrak{K} s^{-1} \aleph \overline{b_{\phi}}$ (see (186a)), differ at $\mathrm{O}\left(\zeta^{3}\right)$. The point here is that, though the functional forms of $\aleph^{\ddagger}$ and $\aleph$ in terms of $\boldsymbol{\zeta}$ and $\boldsymbol{\eta}$ respectively are the same, the values of $\boldsymbol{\zeta}$ and $\boldsymbol{\eta}$ differ at $\mathrm{O}\left(\zeta^{2}\right)$. Following Braginsky (1964a,b), others (see, e.g. Tough 1967, Soward 1971a,b) have extended the definition of effective-variables to higher orders and in that sense our ETHEL-expansion has extended the definition to all orders.

In this section we consider the ETHEL-forms of $\boldsymbol{u}^{*}=\overline{u_{\phi}^{*}} \widehat{\boldsymbol{\phi}}+\boldsymbol{u}^{* \prime}+\overline{\boldsymbol{u}_{\mathrm{m}}^{*}}$ and $\boldsymbol{b}^{*}=\overline{b_{\phi}^{*}} \widehat{\boldsymbol{\phi}}+\boldsymbol{b}^{* \prime}+\overline{\boldsymbol{b}_{\mathrm{m}}^{*}}$ (see $(48 \mathrm{a}-\mathrm{d})$ ) correct to $\mathrm{O}\left(\eta^{3}\right)$. In view of the complicated relation (112b) between $\boldsymbol{\zeta}$ and $\boldsymbol{\eta}$, it is not generally a simple matter to compare the $\boldsymbol{\zeta}$ and $\boldsymbol{\eta}$ expansions. However, for steady flows addressed by Tough (1967), he was able to derive an effective-variable $\boldsymbol{\zeta}$-expansion correct to $\mathrm{O}\left(\zeta^{3}\right)$; though even higher order representations of effective-variables have been obtained (Soward 1971a,b). So our limited objective here is to compare the ETHEL $\boldsymbol{\eta}$-expansions of $\boldsymbol{u}_{\mathrm{m}}$ and $\overline{\boldsymbol{b}_{\mathrm{m}}}$ (see $(\mathrm{G} .16 \mathrm{a}, \mathrm{b})$ ) in terms of $\varpi$ and $\boldsymbol{W}$ (see (G.14a,b)) with Tough's $\boldsymbol{\zeta}$-expansion in in terms of $\varpi^{\ddagger}$ and $\boldsymbol{W}^{\ddagger}$ (see $\left.(\mathrm{G} .11 \mathrm{a}, \mathrm{b})\right)$. Tough also derives $\aleph^{\ddagger}$ correct to $\mathrm{O}\left(\zeta^{3}\right)$ but we do not attempt its comparison with $\aleph$.

To achieve the above objective, it is sufficient to consider the particular case when $\boldsymbol{u}$ and $\boldsymbol{b}$ are azimuthal axisymmetric vectors:

$$
\boldsymbol{u}=u(s, z) \widehat{\boldsymbol{\phi}}, \quad \boldsymbol{b}=b(s, z) \widehat{\boldsymbol{\phi}}
$$

and to reinstate the additional small meridional contributions $\boldsymbol{u}_{\mathrm{m}}, \boldsymbol{b}_{\mathrm{m}}$ in $(154 \mathrm{a}, \mathrm{b})$ later as needed. Then on the basis that $\boldsymbol{\nabla} \cdot \boldsymbol{u}=\boldsymbol{\nabla} \cdot \boldsymbol{b}=\boldsymbol{\nabla} \cdot \boldsymbol{\eta}=0$, we may use (102b), (105b), (98b), where from (99c) $\mathrm{L}_{\boldsymbol{\eta}} \boldsymbol{u} \equiv[\boldsymbol{\eta}, \boldsymbol{u}]=-\boldsymbol{\nabla} \times(\boldsymbol{\eta} \times \boldsymbol{u})$ (likewise for $\boldsymbol{w}$ ) and from (99d) $\mathrm{L}_{\boldsymbol{\eta}} \boldsymbol{b} \equiv \llbracket \boldsymbol{\eta}, \boldsymbol{b} \rrbracket=-\boldsymbol{\nabla} \times(\boldsymbol{\eta} \times \boldsymbol{b})$, to obtain

$$
\begin{aligned}
\boldsymbol{u}^{*}(\boldsymbol{x}, t) & \approx \boldsymbol{u}-\left(-\partial_{t} \boldsymbol{\eta}+\mathrm{L}_{\boldsymbol{\eta}} \boldsymbol{u}\right)+\frac{1}{2} \mathrm{~L}_{\boldsymbol{\eta}}\left(-\partial_{t} \boldsymbol{\eta}+\mathrm{L}_{\boldsymbol{\eta}} \boldsymbol{u}\right)-\frac{1}{3 !} \mathrm{L}_{\boldsymbol{\eta}}\left(-\partial_{t} \boldsymbol{\eta}+\mathrm{L}_{\boldsymbol{\eta}} \boldsymbol{u}\right), \\
\boldsymbol{b}^{*}(\boldsymbol{x}, t) & \approx \boldsymbol{b}-\mathrm{L}_{\boldsymbol{\eta}} \boldsymbol{b}+\frac{1}{2} \mathrm{~L}_{\boldsymbol{\eta}}^{2} \boldsymbol{b}-\frac{1}{3 !} \mathrm{L}_{\boldsymbol{\eta}}^{3} \boldsymbol{b}
\end{aligned}
$$

$\left(\mathrm{L}_{\boldsymbol{\eta}} \bullet=-\boldsymbol{\nabla} \times(\boldsymbol{\eta} \times \bullet)\right)$, taken correct to $\mathrm{O}\left(\eta^{3}\right)$ (see (13c)). The meridional mean parts are

$$
\overline{\boldsymbol{u}_{\mathrm{m}}^{*}}=\nabla \times\left(\left(\overline{\psi_{u}}+\overline{\psi_{u}}\right) \widehat{\phi}\right), \quad \overline{\boldsymbol{b}_{\mathrm{m}}^{*}}=\nabla \times\left(\overline{a_{b}} \widehat{\phi}\right),
$$


where

$$
\begin{aligned}
& \psi_{w} \approx-\frac{1}{2}(\boldsymbol{\eta} \times \widehat{\boldsymbol{\phi}}) \cdot\left(\partial_{t} \boldsymbol{\eta}+\frac{1}{3} \boldsymbol{\nabla} \times\left(\boldsymbol{\eta} \times \partial_{t} \boldsymbol{\eta}\right)\right), \\
& \psi_{u} \approx-\frac{1}{2}(\boldsymbol{\eta} \times \widehat{\boldsymbol{\phi}}) \cdot \boldsymbol{\nabla} \times\left(\boldsymbol{\eta} \times\left(u \widehat{\boldsymbol{\phi}}-\frac{1}{3}[\boldsymbol{\eta}, u \widehat{\boldsymbol{\phi}}]\right)\right), \\
& a_{b} \approx-\frac{1}{2}(\boldsymbol{\eta} \times \widehat{\boldsymbol{\phi}}) \cdot \boldsymbol{\nabla} \times\left(\boldsymbol{\eta} \times\left(b \widehat{\boldsymbol{\phi}}-\frac{1}{3}[\boldsymbol{\eta}, b \widehat{\boldsymbol{\phi}}]\right)\right),
\end{aligned}
$$

in which the naturally arising terms terms linear in $\boldsymbol{\eta}$ have been ignored because their averages needed in $(\mathrm{G} .3 \mathrm{a}, \mathrm{b})$ vanish.

We note that

$$
\boldsymbol{\nabla} \times(\boldsymbol{\eta} \times u \widehat{\boldsymbol{\phi}})=-[\boldsymbol{\eta}, u \widehat{\boldsymbol{\phi}}]=(u / s) \partial_{\phi} \boldsymbol{\eta}-(s \boldsymbol{\eta} \cdot \boldsymbol{\nabla}(u / s)) \widehat{\boldsymbol{\phi}}
$$

which for $u=s$ reduces to

$$
\boldsymbol{\nabla} \times(s \boldsymbol{\eta} \times \widehat{\boldsymbol{\phi}})=\partial_{\phi} \boldsymbol{\eta}
$$

where we recall from (47b) that relative to cylindrical polar coordinates

$$
\left(\partial_{\phi} \boldsymbol{\eta}\right)^{p}=\partial_{\phi} \eta^{p}
$$

Moreover a standard vector identity together with (G.5a-c) yields

$$
\begin{aligned}
\frac{1}{2}(s \boldsymbol{\eta} & \times \widehat{\boldsymbol{\phi}}) \cdot \boldsymbol{\nabla} \times(\boldsymbol{\eta} \times[\boldsymbol{\eta}, u \widehat{\boldsymbol{\phi}}]) \\
& =-\frac{1}{2} \boldsymbol{\nabla} \cdot((s \boldsymbol{\eta} \times \widehat{\boldsymbol{\phi}}) \times(\boldsymbol{\eta} \times[\boldsymbol{\eta}, u \widehat{\boldsymbol{\phi}}]))-\frac{1}{2}(\boldsymbol{\eta} \times[\boldsymbol{\eta}, u \widehat{\boldsymbol{\phi}}]) \cdot(\boldsymbol{\nabla} \times(s \boldsymbol{\eta} \times \widehat{\boldsymbol{\phi}})) \\
& =\boldsymbol{\nabla} \cdot\left(s \Xi_{\phi} \boldsymbol{\eta} u\right)+s^{2} \Xi_{\phi} \boldsymbol{\eta} \cdot \boldsymbol{\nabla}(u / s)=s \boldsymbol{\nabla} \cdot\left(\Xi_{\phi} \boldsymbol{\eta} u\right)+s \Xi_{\phi} \boldsymbol{\eta} \cdot \boldsymbol{\nabla} u,
\end{aligned}
$$

in which we have introduced

$$
\Xi_{t}=-\left(\boldsymbol{\eta} \times \partial_{t} \boldsymbol{\eta}\right)_{\phi} / 2, \quad \Xi_{\phi}=-\left(\boldsymbol{\eta} \times \partial_{\phi} \boldsymbol{\eta}\right)_{\phi} / 2 s .
$$

Aided by the identities (G.5a-c) and (G.6a-c), our relations (G.4a-c) reduce to

$$
\begin{aligned}
& \psi_{w} \approx-\Xi_{t}+\frac{1}{3}\left(s^{-1} \boldsymbol{\nabla} \cdot\left(s \Xi_{t} \boldsymbol{\eta}\right)+\frac{1}{2} s^{-1}\left(\partial_{\phi} \boldsymbol{\eta}\right) \cdot\left(\boldsymbol{\eta} \times \partial_{t} \boldsymbol{\eta}\right)\right), \\
& \psi_{u} \approx-\Xi_{\phi} u+\frac{1}{3}\left(\left(\boldsymbol{\nabla} \cdot\left(\Xi_{\phi} \boldsymbol{\eta}\right)\right) u+2 \Xi_{\phi} \boldsymbol{\eta} \cdot \boldsymbol{\nabla} u\right), \\
& a_{b} \approx-\Xi_{\phi} b+\frac{1}{3}\left(\left(\boldsymbol{\nabla} \cdot\left(\Xi_{\phi} \boldsymbol{\eta}\right)\right) b+2 \Xi_{\phi} \boldsymbol{\eta} \cdot \boldsymbol{\nabla} b\right) .
\end{aligned}
$$

Note that, in the term $\left(\partial_{\phi} \boldsymbol{\eta}\right) \cdot\left(\boldsymbol{\eta} \times \partial_{t} \boldsymbol{\eta}\right)$ of (G.7a), we have $\boldsymbol{\eta}=\boldsymbol{\eta}_{\mathrm{m}}+\eta_{\phi} \widehat{\boldsymbol{\phi}}$. So whereas we may write $\partial_{\phi} \eta_{\phi}=-s \boldsymbol{\nabla} \cdot \boldsymbol{\eta}_{\mathrm{m}}$, there is no corresponding simplification for $\partial_{t} \eta_{\phi}$.

The best interpretation of $\zeta$ in the Braginsky (1964a,b) context is to suppose that the formula (50a) for $\boldsymbol{u}^{* \prime}$ holds exactly with $\boldsymbol{\nabla} \cdot \boldsymbol{\zeta}=0$ and $\overline{\boldsymbol{\zeta}}=\mathbf{0}$ :

$$
\begin{aligned}
\boldsymbol{u}^{* \prime}-\partial_{t} \boldsymbol{\zeta} & =\left(\overline{u_{\phi}^{*}} / s\right) \partial_{\phi} \boldsymbol{\zeta}-\left(s \boldsymbol{\zeta} \cdot \nabla\left(\overline{u_{\phi}^{*}} / s\right)\right) \widehat{\boldsymbol{\phi}} \\
& =\nabla \times\left(\boldsymbol{\zeta} \times \overline{u_{\phi}^{*}} \widehat{\boldsymbol{\phi}}\right)=-\left[\boldsymbol{\zeta}, \overline{u_{\phi}^{*}} \widehat{\boldsymbol{\phi}}\right] \equiv-\mathrm{L}_{\boldsymbol{\zeta}}\left(\overline{u_{\phi}^{*}} \widehat{\boldsymbol{\phi}}\right) .
\end{aligned}
$$

Noting that $\overline{u_{\phi}^{*}} \widehat{\boldsymbol{\phi}} \simeq \overline{\boldsymbol{u}^{*}} \simeq \boldsymbol{u}$ and $\boldsymbol{\zeta} \simeq \boldsymbol{\eta}$, substitution of (111b) into (112b) gives

$$
\partial_{t}(\boldsymbol{\zeta}-\boldsymbol{\eta})-\mathrm{L}_{(\boldsymbol{\zeta}-\boldsymbol{\eta})}\left(\overline{u_{\phi}^{*}} \widehat{\boldsymbol{\phi}}\right) \approx \frac{1}{2}\left(\mathrm{~L}_{\boldsymbol{\eta}}\left(-\partial_{t} \boldsymbol{\eta}+\mathrm{L}_{\boldsymbol{\eta}} \boldsymbol{u}\right)\right)^{\prime} .
$$

In order to compare our results with those of Tough (1967), we restrict attention to steady flow $\left(\partial_{t}=0\right)$ and express (G.9a) in the alternative form

$$
(u / s) \partial_{\phi}(\boldsymbol{\eta}-\boldsymbol{\zeta})-(s(\boldsymbol{\eta}-\boldsymbol{\zeta}) \cdot \boldsymbol{\nabla}(u / s)) \widehat{\boldsymbol{\phi}} \approx \frac{1}{2}(\boldsymbol{\nabla} \times(\boldsymbol{\zeta} \times[\boldsymbol{\zeta}, u \widehat{\boldsymbol{\phi}}]))^{\prime},
$$


where the prime denotes the fluctuating part. From it we obtain

$$
\begin{aligned}
\overline{\widehat{\phi} \cdot\left(\boldsymbol{\eta} \times \partial_{\phi} \boldsymbol{\eta}\right)} & \approx \overline{\boldsymbol{\phi} \cdot\left(\boldsymbol{\zeta} \times \partial_{\phi} \boldsymbol{\zeta}\right)}+2 \overline{\widehat{\boldsymbol{\phi}} \cdot\left(\boldsymbol{\zeta} \times \partial_{\phi}(\boldsymbol{\eta}-\boldsymbol{\zeta})\right)} \\
& \approx \overline{\widehat{\phi} \cdot\left(\boldsymbol{\zeta} \times \partial_{\phi} \boldsymbol{\zeta}\right)}-(s / u) \overline{(\boldsymbol{\zeta} \times \widehat{\boldsymbol{\phi}}) \cdot(\boldsymbol{\nabla} \times(\boldsymbol{\zeta} \times[\boldsymbol{\zeta}, u \widehat{\boldsymbol{\phi}}]))}
\end{aligned}
$$

We introduce the Braginsky (1964a) and Tough (1967) variables $\varpi^{\ddagger}($ see $(51 \mathrm{~g}))$ and $\boldsymbol{W}^{\dagger}$ :

$$
\varpi^{\ddagger}=-\left(\overline{\boldsymbol{\zeta} \times \partial_{\phi} \boldsymbol{\zeta}}\right)_{\phi} / 2 s, \quad \boldsymbol{W}^{\ddagger}=-\overline{\left(\boldsymbol{\zeta} \times \partial_{\phi} \boldsymbol{\zeta}\right)_{\phi} \boldsymbol{\zeta}} / 3 s
$$

respectively. We now utilise the identity (G.6a) with $\boldsymbol{\eta}$ replaced by $\boldsymbol{\zeta}$ and so find

$$
\varpi=\overline{\Xi_{\phi}} \approx \varpi^{\ddagger}+\frac{3}{2}\left(\boldsymbol{\nabla} \cdot \boldsymbol{W}^{\ddagger}+\frac{2}{u}\left(\boldsymbol{W}^{\ddagger} \cdot \boldsymbol{\nabla}\right) u\right)=\varpi^{\ddagger}+\frac{3}{2 u^{2}} \boldsymbol{\nabla} \cdot\left(\boldsymbol{W}^{\ddagger} u^{2}\right) .
$$

In this way we obtain

$$
\begin{aligned}
& \overline{\psi_{u}} \approx-\varpi u+\frac{1}{2 u} \nabla \cdot\left(\boldsymbol{W} u^{2}\right) \approx-\varpi^{\ddagger} u-\frac{1}{u} \nabla \cdot\left(\boldsymbol{W}^{\ddagger} u^{2}\right), \\
& \overline{a_{b}} \approx-\varpi b+\frac{1}{2 b} \boldsymbol{\nabla} \cdot\left(\boldsymbol{W} b^{2}\right) \approx-\varpi^{\ddagger} b-\frac{b}{u^{2}} \boldsymbol{\nabla} \cdot\left(\boldsymbol{W}^{\ddagger} u^{2}\right)+u \boldsymbol{W} \cdot \boldsymbol{\nabla}\left(\frac{b}{u}\right),
\end{aligned}
$$

in which

$$
\varpi=-\left(\overline{\boldsymbol{\eta} \times \partial_{\phi} \boldsymbol{\eta}}\right)_{\phi} / 2 s, \quad \boldsymbol{W}=-\overline{\left(\boldsymbol{\eta} \times \partial_{\phi} \boldsymbol{\eta}\right)_{\phi} \boldsymbol{\eta}} / 3 s \approx \boldsymbol{W}^{\ddagger} .
$$

The $\mathrm{O}\left(\zeta^{2}\right)$ terms proportional to $\varpi^{\ddagger}$ in $(\mathrm{G} .13 \mathrm{a}, \mathrm{b})$ agree with Braginsky (1964a,b), while the $\mathrm{O}\left(\zeta^{3}\right)$ terms linear in $\boldsymbol{W}^{\ddagger}$ agree reassuringly with Tough (1967). The former ETHELrepresentations in (G.13a,b) of $\overline{\psi_{u}}$ and $\overline{a_{b}}$ in terms of $\varpi$ and $\boldsymbol{W}$ are more symmetric than the latter Tough representations in terms of $\varpi^{\ddagger}$ and $\boldsymbol{W}^{\ddagger}$, Indeed the unattractive feature of the Tough formula (G.13b) for $\overline{a_{b}}$ is that it involves $u$ explicitly, whereas the ETHEL-form does not.

Evidently with the inclusion of $\boldsymbol{u}_{\mathrm{m}}=\boldsymbol{\nabla} \times(\psi \widehat{\boldsymbol{\phi}})=\mathrm{O}\left(\eta^{2} u_{\phi}\right)$ and $\overline{\boldsymbol{b}_{\mathrm{m}}}=\boldsymbol{\nabla} \times(a \widehat{\boldsymbol{\phi}})=\mathrm{O}\left(\eta^{2} \overline{b_{\phi}}\right)$ so far neglected, the restricted forms (G.3a,b) generalise to

$$
\begin{aligned}
& \overline{\boldsymbol{u}_{\mathrm{m}}^{*}}=\boldsymbol{\nabla} \times\left(\left(\psi+\left(\overline{\psi_{w}}+\overline{\psi_{u}}\right)\right) \widehat{\phi}\right)+\mathrm{O}\left(\eta^{2} \boldsymbol{u}_{\mathrm{m}}\right), \\
& \overline{\boldsymbol{b}_{\mathrm{m}}^{*}}=\boldsymbol{\nabla} \times\left(\left(a+\overline{a_{b}}\right) \widehat{\boldsymbol{\phi}}\right)+\mathrm{O}\left(\eta^{2} \overline{\boldsymbol{b}_{\mathrm{m}}}\right),
\end{aligned}
$$

where, in the definitions (G.13a,b) of $\overline{\psi_{u}}$ and $\overline{a_{b}}, u$ and $b$ are replaced by $u_{\phi}$ and $\overline{b_{\phi}}$ respectively. So whereas (G.15a,b) defines the Eulerian means in terms of HEL-variables, they may be inverted to provide the expressions

$$
\begin{aligned}
& \boldsymbol{u}_{\mathrm{m}}=\nabla \times\left(\left(\psi^{*}-\left(\overline{\psi_{w}}+\overline{\psi_{u}}\right)\right) \hat{\boldsymbol{\phi}}\right)+\mathrm{O}\left(\zeta^{2} \overline{\boldsymbol{u}_{\mathrm{m}}^{*}}\right), \\
& \overline{\boldsymbol{b}_{\mathrm{m}}}=\boldsymbol{\nabla} \times\left(\left(a^{*}-\overline{a_{b}}\right) \widehat{\phi}\right)+\mathrm{O}\left(\zeta^{2} \overline{\boldsymbol{b}_{\mathrm{m}}^{*}}\right)
\end{aligned}
$$

for the HEL-variables in terms of Eulerian means. In the spirit of the equivalence (114) of HEL and effective variables, (G.16a,b) provide an improved approximation on the lowest order Braginsky (1964a,b) representations (51a-d) of the effective-variables $\boldsymbol{u}^{\dagger}$ and $\overline{\boldsymbol{b}^{\dagger}}$.

We provide a final word of caution. For, whereas our original definition of the fluctuating velocity $\boldsymbol{u}^{* \prime}=\partial_{t} \boldsymbol{\zeta}-\left[\boldsymbol{\zeta}, \overline{\boldsymbol{u}^{*}}\right]$ (see (9a)), which we used in our introductory sections 1.2 and 1.3 was based on the entire mean velocity $\overline{\boldsymbol{u}^{*}}=\overline{u_{\phi}^{*}} \widehat{\boldsymbol{\phi}}+\overline{\boldsymbol{u}_{\mathrm{m}}^{*}}$, our development in this section has hinged on $\boldsymbol{u}^{* \prime}=\partial_{t} \boldsymbol{\zeta}-\left[\boldsymbol{\zeta}, \overline{u_{\phi}^{*}} \widehat{\boldsymbol{\phi}}\right]$ (see (G.8)). The discrepancy between the definitions is of no consequence either at the quadratic order of accuracy $\mathrm{O}\left(\zeta^{2}\right)$ demanded in the introductory section 1.4 or at the cubic order of accuracy $\mathrm{O}\left(\zeta^{3}\right)$ attempted in this section. However, when 
the error terms in (G.16a,b) are determined at the next quartic level $\mathrm{O}\left(\zeta^{4}\right)$, the term $-\left[\boldsymbol{\zeta}, \overline{\boldsymbol{u}_{\mathrm{m}}^{*}}\right]$ omitted in (G.8) needs to be reinstated if the strategy envisaged in section 1 is to be pursued. Really, this is a distraction, because different strategies involving alternative definitions of the auxiliary variable $\boldsymbol{\zeta}$ may be both legitimate and useful (e.g. $\boldsymbol{\eta}$ itself!). Our main point is that effective-variables defined via ETHEL $\boldsymbol{\eta}$ rather than $\boldsymbol{\zeta}$ are preferable because of their unambiguous Lagrangian interpretation as well as the fact that the ETHEL-expansions are simply Taylor series (see $(98 \mathrm{a}, \mathrm{b}))$ that generalise readily to higher orders. 